Florida International University

FIU Digital Commons

FIU Electronic Theses and Dissertations

University Graduate School

$3-27-2015$

\title{
Fiber Scaffolds of Poly (glycerol-dodecanedioate) and its Derivative via Electrospinning for Neural Tissue Engineering
}

Xizi Dai

Florida International University, xdai001@fiu.edu

DOI: $10.25148 /$ etd.FI15032147

Follow this and additional works at: https:// digitalcommons.fiu.edu/etd

Part of the Biomaterials Commons, and the Molecular, Cellular, and Tissue Engineering Commons

\section{Recommended Citation}

Dai, Xizi, "Fiber Scaffolds of Poly (glycerol-dodecanedioate) and its Derivative via Electrospinning for Neural Tissue Engineering" (2015). FIU Electronic Theses and Dissertations. 1852.

https://digitalcommons.fiu.edu/etd/1852

This work is brought to you for free and open access by the University Graduate School at FIU Digital Commons. It has been accepted for inclusion in FIU Electronic Theses and Dissertations by an authorized administrator of FIU Digital Commons. For more information, please contact dcc@fiu.edu. 


\section{FLORIDA INTERNATIONAL UNIVERSITY}

Miami, Florida

\section{FIBER SCAFFOLDS OF POLY (GLYCEROL- DODECANEDIOATE) AND ITS DERIVATIVE VIA ELECTROSPINNING FOR NEURAL TISSUE ENGINEERING}

A dissertation submitted in partial fulfillment of the

requirements for the degree of

DOCTOR OF PHILOSOPHY

in

BIOMEDICAL ENGINEERING

by

Xizi Dai

2015 
To: Dean Amir Mirmiran

College of Engineering and Computing

This dissertation, written by Xizi Dai, and entitled Fiber Scaffolds of Poly (glycerol-dodecanedioate) and its Derivative via Electrospinning for Neural Tissue Engineering, having been approved in respect to style and intellectual content, is referred to you for judgment.

We have read this dissertation and recommend that it be approved.

Anthony J. McGoron

Lidia Kos

Nikolaos Tsoukias

Wei-Chiang Lin, Major Professor

Date of Defense: March 27, 2015

The dissertation of Xizi Dai is approved.

Dean Amir Mirmiran

College of Engineering and Computing

Dean Lakshmi N. Reddi

University Graduate School

Florida International University, 2015 


\section{DEDICATION}

I would like to dedicate this dissertation to my husband Adnan Ismail, my parents, my uncle and aunt, who have been fully supportive and helped me throughout this journey. I want to thank my husband, with whom, as newly-weds, I navigated the last year of my Ph.D. Thanks to your support and encouragement, I have been able to overcome both expected and unexpected obstacles during the last few years. Thank you for being my human spell-check, for being by my side every weekend in the lab, and for always knowing what to do and say when the going gets tough. I want to thank my parents who brought me into this world and have been always there for me. Whose many sacrifices for my sake, their only child, can never be repaid. Who supported my decision, even when it pained them, as long as it made me happy. I would also like to thank my uncle and aunt, who supported me and stood by me after my operation, and where my shining light during the darkest days of my life in the United States. I cannot express my gratitude for having them by my side, when I was most lonely and helpless, and I will never forget they were here for me when I needed them.

No one says that the life of a Ph.D. candidate is an easy one, and I came to understand why. There were so many moments when I just wanted to give up and walk away, but thanks to the support of my friends and family, I persevered. Looking back at the past five years of my life, I realize that they offered me the most valuable experience of my life, not only because they led to a doctoral degree, but mainly because they taught me how to solve problems, how to work with people, how to deal with stress, and how to never give up. 


\section{ACKNOWLEDGMENTS}

I would like to acknowledge my mentor Dr. Yen-Chih Huang for his guidance and mentoring through all these years. He taught me how to become a real scientist and showed me the right attitude toward science. He has always had my best interest at heart and provided me with every opportunity to learn. I really appreciate all the patience and kindness that he has shown me over these past five years, even after he left the department. I also want to thank Dr. WeiChiang Lin, who took over as my advisor following Dr. Huang's departure, for his great support in the last year of my Ph.D. career. Without his help, there is no way I could have finished my experiments and graduate on time. I would also like to thank my dissertation committee members Dr. Anthony J. McGoron, Dr. Lidia Kos and Dr. Nikolaos Tsoukias for their invaluable feedback and advice to steer me in the right direction of my research and improve my dissertation. I want to thank the lab manager Yun Qian, who helped me a lot with managing the lab

supplies during the last two semesters. I also want to thank my labmates Jared Leichner, Emily Chereson and Anh Lee, who rallied to my help during the difficult period following Dr. Huang's departure.

I would also like to acknowledge the financial support and funding during my Ph.D. studies provided by a Teaching Assistantship from the Biomedical Engineering Department at FIU from 2009-2015 that made this moment possible. I truly thank all of you from the bottom of my heart. 


\begin{abstract}
OF THE DISSERTATION
FIBER SCAFFOLDS OF POLY (GLYCEROL- DODECANEDIOATE) AND ITS

DERIVATIVE VIA ELECTROSPINNING FOR NEURAL TISSUE ENGINEERING

by
\end{abstract}

Xizi Dai

Florida International University, 2015

Miami, Florida

Professor Wei-Chiang Lin, Major Professor

Peripheral nerves have demonstrated the ability to bridge gaps of up to 6 $\mathrm{mm}$. Peripheral Nerve System injury sites beyond this range need autograft or allograft surgery. Central Nerve System cells do not allow spontaneous regeneration due to the intrinsic environmental inhibition. Although stem cell therapy seems to be a promising approach towards nerve repair, it is essential to use the distinct three-dimensional architecture of a cell scaffold with proper biomolecule embedding in order to ensure that the local environment can be controlled well enough for growth and survival. Many approaches have been developed for the fabrication of $3 \mathrm{D}$ scaffolds, and more recently, fiber-based scaffolds produced via the electrospinning have been garnering increasing interest, as it offers the opportunity for control over fiber composition, as well as fiber mesh porosity using a relatively simple experimental setup. All these attributes make electrospun fibers a new class of promising scaffolds for neural tissue engineering. Therefore, the purpose of this doctoral study is to investigate the use of the novel material PGD and its derivative PGDF for obtaining fiber 
scaffolds using the electrospinning. The performance of these scaffolds, combined with neural lineage cells derived from ESCs, was evaluated by the dissolvability test, Raman spectroscopy, cell viability assay, real time PCR, Immunocytochemistry, extracellular electrophysiology, etc. The newly designed collector makes it possible to easily obtain fibers with adequate length and integrity. The utilization of a solvent like ethanol and water for electrospinning of fibrous scaffolds provides a potentially less toxic and more biocompatible fabrication method. Cell viability testing demonstrated that the addition of gelatin leads to significant improvement of cell proliferation on the scaffolds. Both real time PCR and Immunocytochemistry analysis indicated that motor neuron differentiation was achieved through the high motor neuron gene expression using the metabolites approach. The addition of Fumaric acid into fiber scaffolds further promoted the differentiation. Based on the results, this newly fabricated electrospun fiber scaffold, combined with neural lineage cells, provides a potential alternate strategy for nerve injury repair. 


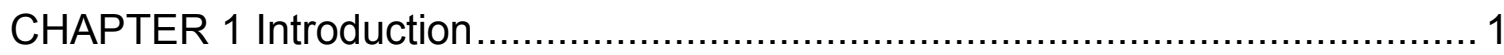

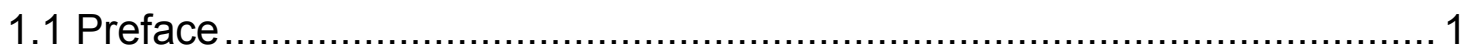

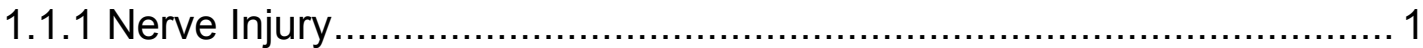

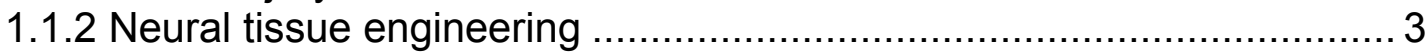

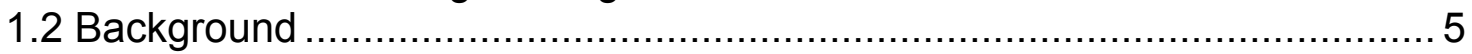

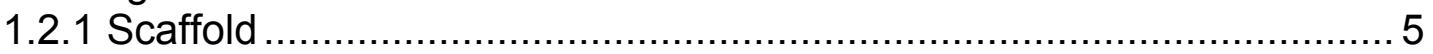

1.2.1.1 Poly (glycerol- dodecanedioate) ….......................................... 5

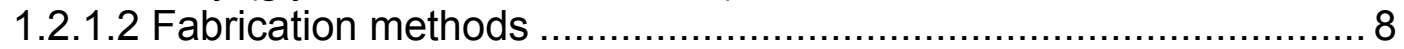

1.2.2 Stem cells and its differentiated neurons ...................................... 15

1.2.2.1 Teratomas, pluripotent stem cells, and mouse embryonic stem

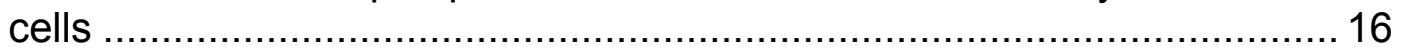

1.2.2.2 Human embryonic stem cells ............................................. 16

1.2.2.3 Induced pluripotent stem cells ............................................ 18

1.2.2.4 Neural stem cells, neurons and glial cells ................................ 19

1.2.2.5 Motor neuron .................................................................. 21

1.3 Current research of cellular interactions on electrospun scaffolds for

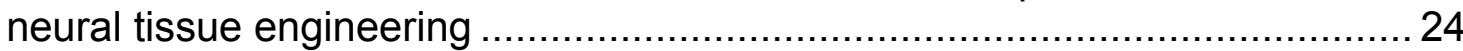

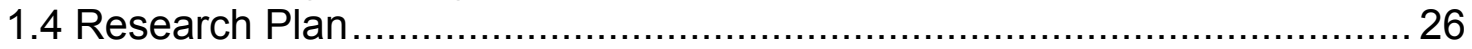

CHAPTER 2 Efficient and Low-Cost Differentiation of Embryonic Stem Cells into Motor Neurons by Metabolites for Neural Tissue Engineering .....................29

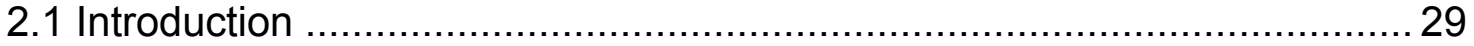

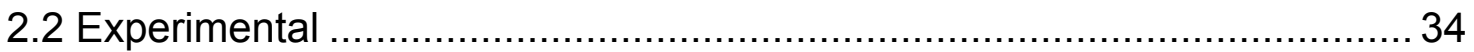

2.2.1 Motor Neuron Induction from Mouse Embryonic Stem cells .............. 34

2.2.2 Functional evaluation of motor neuron differentiation......................... 35

2.2.2.1 Reverse Transcription-Polymerase Chain Reaction .................... 35

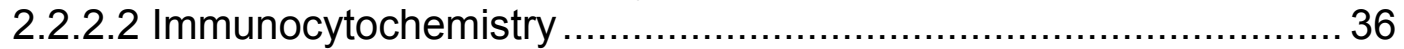

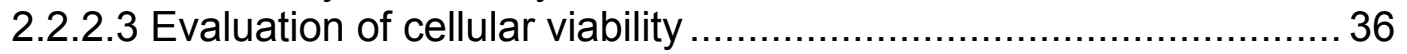

2.2.2.4 Extracellular Electrophysiology ............................................. 37

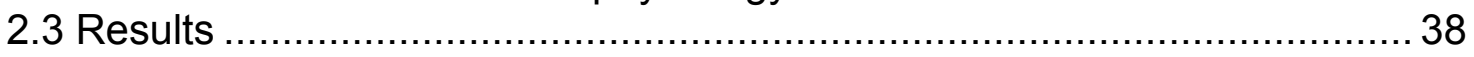

2.3.1 Efficient motor neuron induction from embryonic stem cells ............... 38

2.3.1.1 Schematic procedures of motor neuron differentiation ................. 38

2.3.1.2 RA Increases the Cell Viability and Restricts ESCs into Neuronal

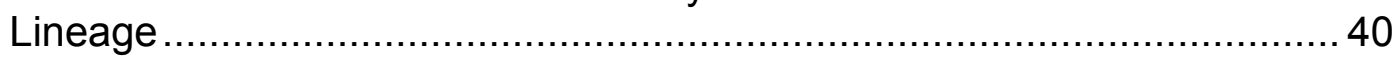

2.3.1.3 The Effect of $\mathrm{NaB}$ on the Conversion of ESCs into Neural Stem Cells

2.3.1.4 Continued Presence of Genistein and NaF Promotes Motor

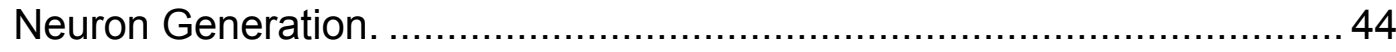

2.3.1.5 Effects of Various Combinations of Three Metabolites ................ 47

2.3.1.6 Purmorphamine Replaces SHH for Motor Neuron Differentiation. 48

2.3.1.7 Functional Features of ESC derived Motor Neuron. .................... 50 
CHAPTER 3 Development of Electrospun Fiber Scaffolds using Poly (glyceroldodecanedioate)/Gelatin Blended for Neural Tissue Engineering .....................6 60

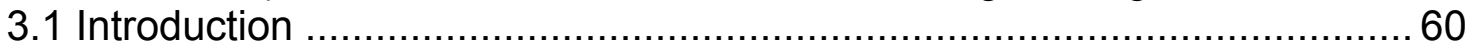

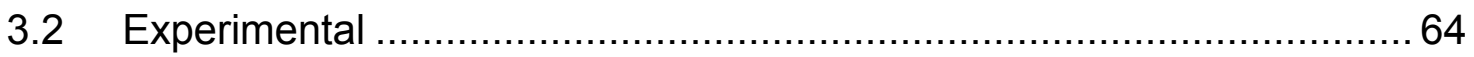

3.2.1 Determination of PGD molecular weight by Gel Permeation

Chromatography (GPC)/Size Exclusion Chromatography (SEC)................64

3.2.2 Preparation of $P G D / g e l a t i n$ polymer solution and fabrication of

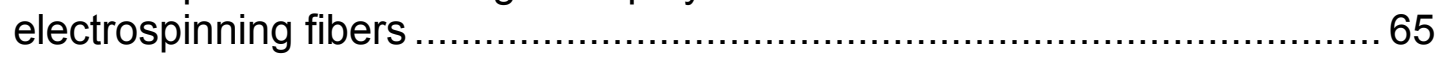

3.2.3 Characterization of PGD/gelatin fiber scaffolds.............................. 67

3.2.3.1 Scanning electron microscopy (SEM) of Electrospun fibers .........6 67

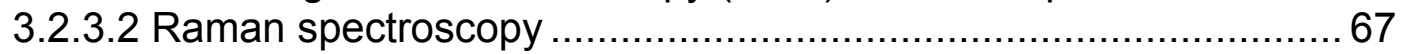

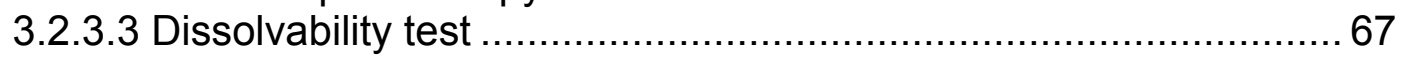

3.2.4 In vitro studies of PGD/gelatin fibers with human foreskin fibroblasts. 68

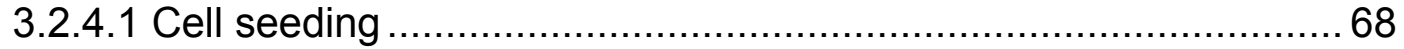

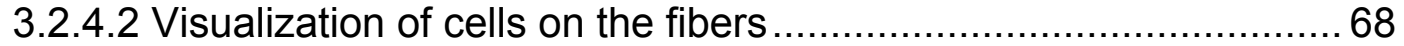

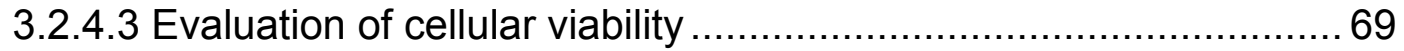

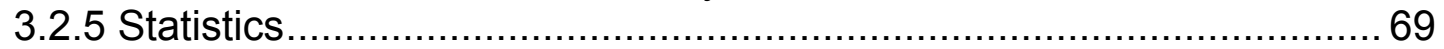

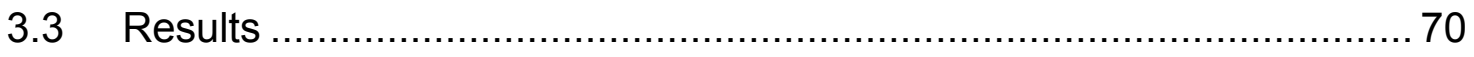

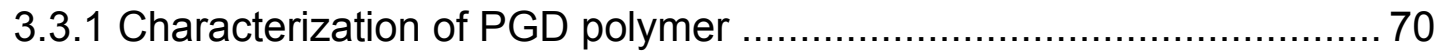

3.3.1.1 Determination of PGD molecular weight ................................ 70

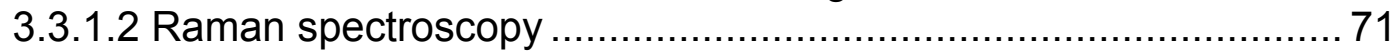

3.3.2 Characterization of PGD/gelatin electrospun fibers ......................... 72

3.3.2.1 The newly designed collector and the effects of PGD

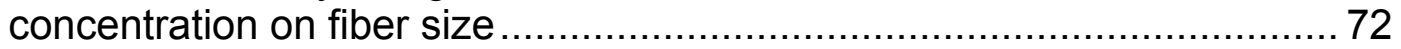

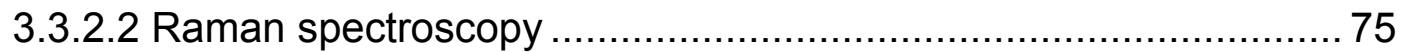

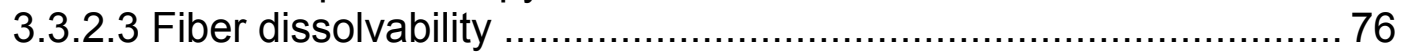

3.3.3 In vitro studies of PGD/gelatin fiber scaffolds ................................ 77

3.3.3.1 Visualization of cells on fiber scaffolds ..................................... 77

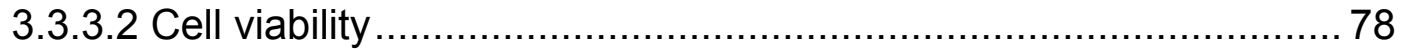

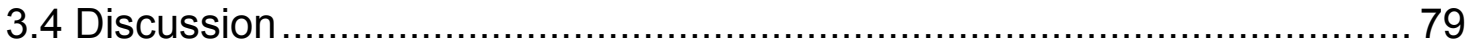

CHAPTER 4 Modification of Electrospun Fiber Scaffolds Composed of PGDF

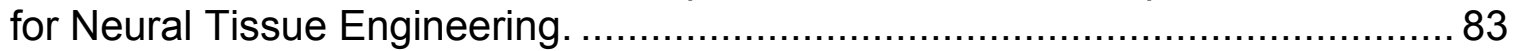

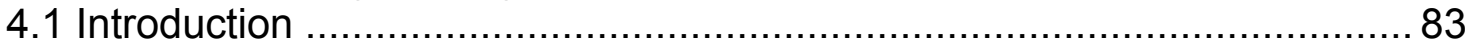

4.1.1 Embedding Biomolecules into fiber Scaffolds for Neural Tissue

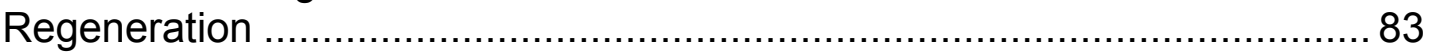

4.1.2 Functions of poly (glycerol- dodecanedioate co-fumarate)................. 84

4.1.3 Peptides modification on the synthetic polymers ............................. 86

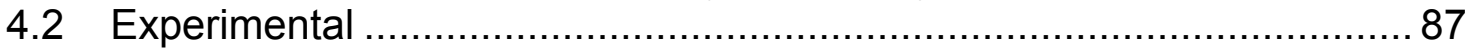

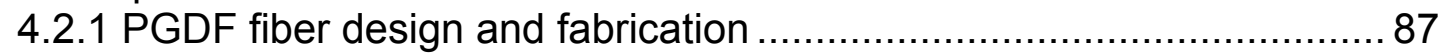

4.2.2 Characterization of PGDF fiber scaffolds ........................................ 89

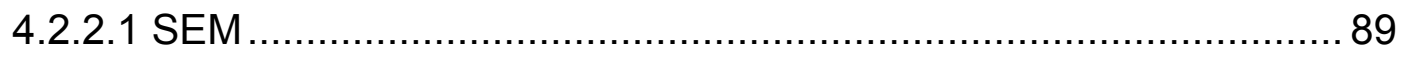

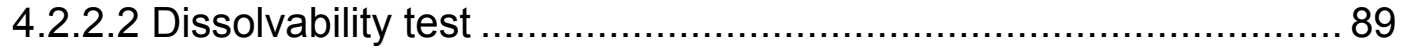


4.2.2.3 FA Release Behaviors of Electrospun fiber ............................... 89

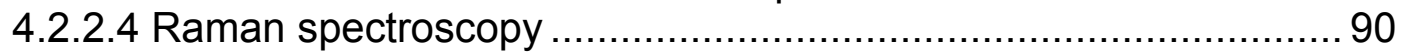

4.2.2.5 Peptides Immobilization .......................................................... 90

4.2.3 In vitro studies of PGDF fibers with mouse embryonic stem cells ....... 90

4.2.3.1 Cell seeding and distribution ................................................... 90

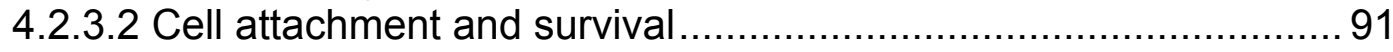

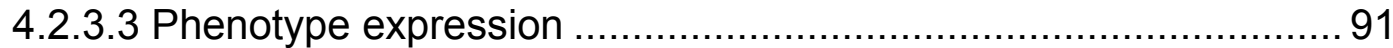

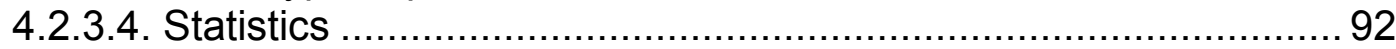

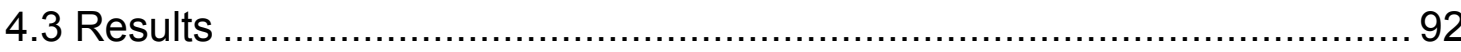

4.3.1 Characterization of PGDF electrospun fibers ................................ 92

4.3.1.1 Fabrication of scaffolds with different fiber diameter .................. 92

4.3.1.2 Electrospun fiber dissolvability ............................................. 94

4.3.1.3 Fumaric acid release profile from electrospun fibers .................. 95

4.3.1.4 Raman spectroscopy .......................................................... 96

4.3.2 Effect of peptides immobilized fibers on cell viability ........................ 98

4.3.2.1 UV detection of peptides binding ........................................... 98

4.3.2.2 Cell viability within the scaffold ............................................. 99

4.3.2.3 Cell differentiation on different fiber diameter and fiber

immobilized with peptides .............................................................. 100

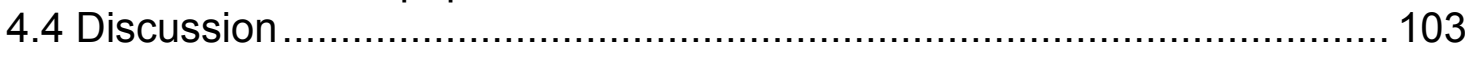

CHAPTER 5 Conclusions and Future Work ............................................. 106

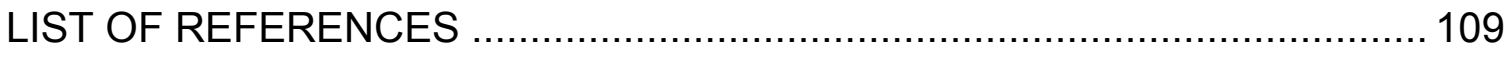

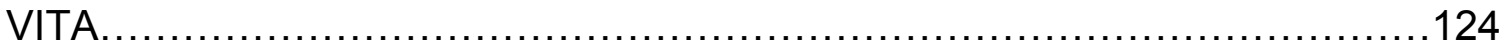




\section{LIST OF FIGURES}

FIGURE

PAGE

Figure 2.1. Motor neuron differentiation and morphology of mouse embryonic stem cells.

Figure 2.2. Effects of RA introduction on mRNA expression, transcription factors and viability.

Figure 2.3. NaB treatment induces neural stem cells differentiation of embryonic stem cells.

Figure 2.4 Continued presence of Genistein promotes motor neuron generation.

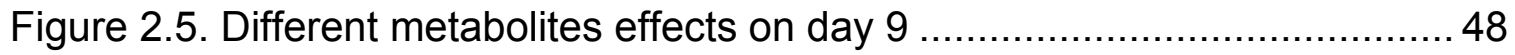

Figure 2.6. Efficient generation of motor neurons by PUR ............................. 49

Figure 2.7. Electrophysiological analysis of motor neurons ............................. 53

Figure 3.1. Synthetic biodegradable elastomer, poly (glycerol-dodecanedioate).

Figure 3.2. Electrospinning setup including a syringe pump, a high voltage power supply and a new designed collector.

Figure 3.3. Number and weight average molecular weight determinations of PGD.

Figure 3.4. Raman spectra of DDA, glycerol and PGD, respectively ................ 72

Figure 3.5. A newly designed collector for electrospinning ............................. 74

Figure 3.6. SEM images and diameter distribution of electrospun PGD fibers from (A) $30 \%$, (B) $40 \%$, and (C) $50 \%(\mathrm{w} / \mathrm{w})$ polymeric solution ....................... 75

Figure 3.7. Raman spectra of PGD/BS fibers prior to and after electrospinning. 76

Figure 3.8. Surface morphology of the degraded PGD with gelatin fibers after different time points. PGD with gelatin fibers after (A) 4 days; (B) 14 days and (C) 28 days

Figure 3.9. Cell adhesion on the PGD with gelatin fibers after 1 day culture. HFF cells staining with Calcein AM reagents (bright green, arrows) on the scaffold $(A)$ and tissue culture dish (B). 
Figure 3.10. Resazurin assay showing the cell viability on PGD with Gelatin fibers and PGD without gelatin fibers after 1, 3, 5, and 8 days. Cells directly cultured on the tissue culture dishes used as control.

Figure 4.1. (A) Synthetic biodegradable elastomer, poly (glycerol -dodecanedioate co-fumarate). (B) RGDC immobilization.

Figure 4.2. SEM images (A-D) and diameter distribution (E-H) of electrospun PGDF fibers from (A,E) $30 \%,(B, F) 40 \%$, and $(C, G) 50 \%$ and $(D, H) 60 \%(w / w)$ polymeric solution.

Figure 4.3. Surface morphology of PGDF fibers on (A) Day 1, (B) Day 14, (C)

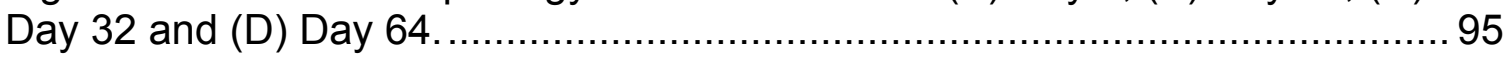

Figure 4.4. Releasing profile of FA from PGDF electrospun fibers................... 96

Figure 4.5. Raman spectra of PGDF and PGD, respectively......................... 97

Figure 4.6. Concentration of double bonded FA in PGDF fiber with and

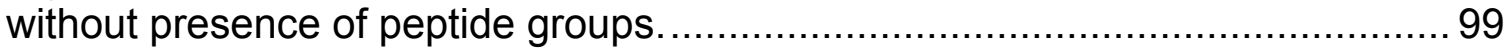

Figure 4.7. (A) Resazurin assay showing the cell viability on PGDF and peptides modified PGDF fibers on day 4, 6, 8, and 11 . 100

Figure 4.8. Expression of neural lineage markers through real time-PCR....... 102 


\section{ABBREVIATIONS AND ACRONYMS}

ALS Amyotrophic lateral sclerosis

AMPK AMP-activated protein kinase

ALK5 Activin receptor-like kinase 5

BMP Bone morphogenetic protein

BDNF Brain-derived neurotrophic factor

BS Basal solution

CNS Central nerve system

CVA Cerebrovascular accident

CS Chondroitin sulfate

ChANS Chondroitinase ABC

CHIR CHIR99021

DDA Dodecanedioic acid

DM Dorsomorphin

DMEM Dulbecco's Modified Eagle's Medium

DAPI 4', 6'-diamidino-2-phenylindole

DAQ Data acquisition

EC Embryonal carcinoma

EGF Epidermal growth factor

ECM Extracellular matrix

ELSD Evaporative Light Scattering Detector

FGF2 Fibroblast growth factor-2

FBS Fetal bovine serum 


$\begin{array}{ll}\text { FA } & \text { Fumaric acid } \\ \text { GFP } & \text { Green fluorescent protein } \\ \text { GM } & \text { Growth medium } \\ \text { GDNF } & \text { Glial cell-derived neurotrophic factor } \\ \text { GAPDH } & \text { Glyceraldehydes-3-phosphate dehydrogenase } \\ \text { GPC } & \text { Gel Permeation Chromatography } \\ \text { hESCs } & \text { Human embryonic stem cells } \\ \text { HDAC } & \text { Histone deacetylase } \\ \text { HPLC } & \text { High Performance Liquid Chromatography } \\ \text { HFF } & \text { Human foreskin fibroblasts } \\ \text { IPSCs } & \text { Induced pluripotent stem cells } \\ \text { LIF } & \text { Leukemia inhibitory factor } \\ \text { MiRNA } & \text { microRNA } \\ \text { mESCs } & \text { Mouse embryonic stem cells } \\ \text { mTG } & \text { microbial transglutaminase } \\ \text { mTOR } & \text { Mammalian target of rapamycin } \\ \text { MW } & \text { Molecular weight } \\ \text { NSCs } & \text { Neural stem cells } \\ \text { NPCs } & \text { Neural progenitor cells } \\ \text { NT-3 } & \text { Neurotrophin-3 } \\ \text { NIM } & \text { Neural induction medium } \\ \text { NaF } & \text { Sodium Fumarate } \\ \text { Sodium Butyrate }\end{array}$


PNS Peripheral nerve system

PLA Poly (lactic acid)

PGA Poly (glycolic acid)

PGD Poly (glycerol-dodecanedioate)

PGS Poly (glycerol sebacate)

PMMA Poly (methyl methacrylate)

PEO Polyethylene oxide

PVA Poly (vinyl alcohol)

PLLA Poly (L-lactic acid)

PCL Poly ( $\varepsilon$-caprolactone)

PLGA Poly (D,L-lactic acid- co- glycolic acid)

PANI Polyaniline

PPy Polypyrrole

PGDF Poly (glycerol dodecanedioate co-fumarate)

PLCL Poly (L-lactic acid)-co-poly (e-caprolactone)

PUR Purmorphamine

PCR Polymerase Chain Reaction

PBS Phosphate buffered saline

RA Retinoic acid

RGD Arginyl-glycyl-aspartic

SCNT Somatic Cell Nuclear Transfer

$\mathrm{SHH} \quad$ Sonic hedgehog

SMA Spinal muscular atrophy 
SB $\quad$ SB431542

SEC Size Exclusion Chromatography

SEM Scanning Electron Microscopy

TBI Traumatic brain injury

TGF- $\beta$ Transforming growth factor-beta

TCA Tricarboxylic acid

Tuj $\quad \beta$-Tubulin III

THF Tetrahydrofuran 
CHAPTER 1 Introduction

\subsection{Preface}

\subsubsection{Nerve Injury}

The nervous system can be divided into two major sections: the central nerve system (CNS), which consists of the brain and the spinal cord, and the peripheral nerve system (PNS), which consists of the nerves that extend to the rest of the body from the CNS [1]. The CNS injury is usually caused by a stroke, a traumatic brain injury or developmental complications [2]. A stroke, also referred to as cerebrovascular accident (CVA), is the loss of brain function due to a disturbance (either ischemia or hemorrhage) in the blood supply to the brain [3]. Usually, it causes part of the brain to function improperly, leading to potentially severe consequences such as vision impairment, aphasia, or inability to move one or more limbs [4]. Traumatic brain injury (TBI) happens when the brain is traumatically injured by an external force. It is a major cause of death and disability worldwide, and it is usually caused by accidental falls, violence or accidents [5]. In case of developmental complications, when CNS is not properly developed resulting in abnormal tissue growth, the function of the CNS will be highly affected, leading to neurological disorders [6]. The major cause of PNS injury is trauma [1]. Fragile nerves can be damaged by stretching, pressure or cutting, splitting the nerve into proximal and distal sections. When a nerve is damaged by stretching or pressure, the nerve fibers break while the surrounding tissues, which can be seen as an insulation, may remain intact. The proximal section is still viable while the distal section dies. After a period, the damaged 
nerve may start the regeneration process. New nerve fibers may grow under the surrounding tissue until they reach the sensory receptor or muscle. When a nerve is cut, both the nerve and the surrounding tissues are severed. Without proper medical treatment, the growing nerve fibers could form a painful nerve scar, called neuroma. PNS injury interferes with signal transmission from the body to the spinal cord and the brain, causing loss of muscle functions, and loss of sensation in the injured area [7].

Numerous researchers are attempting to find an effective way to treat nerve injuries. For CNS, spontaneous regeneration is quiet limited due to its own inhibitory environment [1]. These inhibitions emerge from the formation of glial scars and myelin-associated inhibitor factors. Therefore, researches are focusing on how to promote the regeneration ability of neural tissues. Delivering molecules such as morphogens, pharmaceutical drugs and microRNA (miRNA) to the injury site is a possible method. Neurogenesis has been seen in some animal studies but the benefits are limited due to the challenge of keeping the molecules present at the injury site at a specific and constant concentration [6]. Another treatment option is stem cell therapy. Briefly, it is the culturing of stem cells in vitro and then transferring these cells into the injury location. This method has the benefit of preventing glial scar formation and promoting neurogenesis originating from the patient, but also runs the risk of tumor development, low survivability of the stem cells and stem cell migration out of the injury site [1]. As for the PNS injuries, they are usually repaired by using either an autograft or an allograft. While this treatment procedure frequently provides a superior neural 
functional recovery, it is also associated with significant drawbacks. For instance, the size mismatch between the recipient and donor nerves, the functional loss at the donor site, and the need of multiple surgeries are the common accompanying drawbacks of autografts [8]. For allografts, patients are exposed to the risk of immune/disease-related complications $[8,9]$. It seems that no viable method to effectively treat neural injuries exists. Researchers are looking for new strategies to change the status-quo.

\subsubsection{Neural tissue engineering}

Tissue engineering has emerged as an excellent approach for regeneration of damaged tissue, with the potential to circumvent the limitations of current treatments [10]. Tissue engineering uses a combination of cells and biomaterials (scaffolds) to repair, maintain, or improve tissue function. Neural tissue engineering is a specific sub-field of tissue engineering. It is primarily a search for strategies to develop effective neural guidance aimed at bridging gaps in damaged peripheral or central neurons [1]. It delivers molecules to the injured nerves by embedding the molecules into the biomaterials; improves the stem cell survivability in vivo by supporting the cells growing at the injury sites; stops cells from migrating out by holding them within the scaffolds. Also, it does not have the side effects (size mismatch and function loss at the donor site) associate with autografts and limits the risk of immune/disease-related complications associated with allografts.

A critical element in successfully producing functional tissue in vitro is to identify suitable biomaterials and use it to fabricate 3-D scaffolds that are 
conducive to cell attachment, proliferation, differentiation and ultimately new tissue formation. Many methodologies have been developed to fabricate 3D scaffolds using biopolymers over the past [11]. Recently, micro/nanofiber-based scaffolds produced by using the electrospinning technique have been garnering increasing interest, as it offers an opportunity to control over the composition of the fibers, as well as over the porosity of the fiber meshes using a relatively simple experimental setup [12]. In additional to its high porosity and large surface area, a electrospun fiber scaffold provides a hierarchical structure highly resemble the extracellular matrix (ECM), an environment critical for cell survival, differentiation, signal transduction and nutrient/waste transport [13]. Moreover, electrospun fibers can be modified with different physical or chemical methods to encapsulate or attach bioactive molecules such as native ECM proteins to support and guide both the survival and differentiation of cells seeded on the scaffold. These attributes make electrospun nanofibers a new class of promising scaffolds especially for neural tissue engineering.

The ultimate goal of this dissertation is to demonstrate that electrospun fiber scaffolds made from PGD and its modified derivative are able to support ESCs survival and differentiation into functional motor neurons using a newly developed protocol. To achieve this goal, a new class of electrospun fiber scaffold needs to be fabricated using a novel material poly (glyceroldodecanedioate) (PGD) and its derivative poly (glycerol dodecanedioate cofumarate) (PGDF). The performance of these fiber scaffolds needs to be evaluated using human foreskin fibroblast cells and, more importantly, neural 
lineage cells derived from embryonic stem cells (ESCs). The outcomes of the latter evaluation, if successful, will form a critical base for the development of an improved strategy for treating peripheral nerve injury.

\subsection{Background}

\subsubsection{Scaffold}

As mentioned before, tissue engineering involves three important elements: viable cells, biomolecules and a structural scaffold. The scaffold serves as a foundation for the cells to grow and differentiate, and for the tissue to form. Different subtypes of tissue engineering require different biomaterials for tissue growing. Currently widely used biodegradable polymers such as poly (lactic acid) (PLA) [14], poly (glycolic acid) (PGA) $[15,16]$ and their copolymers are exceptionally stiff materials. For neural tissue engineering application, biodegradable elastic materials are desired in order to better match the mechanical properties of neural tissues. Therefore, a biodegradable polyester elastomer called poly (glycerol-dodecanedioate) (PGD) was developed by thermal condensation of glycerol and dodecanedioic acid (DDA) to meet this goal.

\subsubsection{Poly (glycerol- dodecanedioate)}

In the human body, cells efficiently build biological polymers from basic units such as nucleotides, amino acids and monosaccharides with the help of various enzymes. These nature polymers degrade back to original building units and could be metabolized in what is called the recyclable process. To efficiently produce a biocompatible and environmentally friendly polymer for tissue engineering, it makes sense to model after the natural process by utilizing 
renewable and green starting materials with minimum waste generation, and performing the reaction in a relatively efficient way without using toxic solvents, hazardous chemicals and energy [17].

The recent surge of interest in thermally crosslinked elastomeric polymers is largely due to the pioneering work of Robert Langer's group, which in 2002 introduced poly (glycerol sebacate) (PGS), a polyester which is analogous to vulcanized rubber [18]. Similar to PGS, Migneco et al successfully developed poly (glycerol dodecanedioate) (PGD) by thermal condensation of glycerol and dodecanedioic acid and demonstrated its unique shape memory property [19, 20]. PGD exhibits elastic properties similar to rubber and is biodegradable; therefore it is very suitable for soft tissue engineering applications. Dodecanedioic acid is a twelve-carbon substance with two terminal carboxylic groups, HOOC $(\mathrm{CH} 2)_{10} \mathrm{COOH}$. Even numbered dicarboxylic acids like sebacic acid and dodecanedioic acid can be metabolized to acetyl-CoA and enter the tricarboxylic acid (TCA) / (citric acid) cycle. Furthermore, the metabolic products of dicarboxylic acids, succinyl-CoA, is a gluconeogenetic precursor and TCA cycle intermediate [21]. Thus, some studies suggest that they could be utilized as alternative fuel substrate for enteral and parenteral nutrition, especially in pathological conditions. The most significant difference between sebacic and dodecanedioic acids is that sebacic acid will be quickly excreted from the kidneys, while dodecanedioic acid will not and is more suitable for the delivery of energy in enteral and parenteral nutrition [22]. 
Furthermore, PGD has one main characteristic that makes it unique compared to PGS. The glass transition temperature of PGD is $32{ }^{\circ} \mathrm{C}$, which is above room temperature but below the physiological temperature. If PGD is deformed below the $T_{g}$, the material will be able to keep its new dimension resulting from the strain. However, if the elongation is induced above the $T_{g}$, the material will immediately recover its original shape. As a consequence, mechanical properties varied significantly based on temperature change. When temperature is above the $T_{g}\left(37^{\circ} \mathrm{C}\right), \mathrm{PGD}$ achieved a Young's modulus, rupture strain, and UTS of $1.08 \mathrm{MPa}, 123 \%$ and $0.45 \mathrm{MPa}$, respectively. However, at room temperature, PGD displayed a vastly different mechanical profile: a Young's modulus of $135.55 \mathrm{MPa}$, a rupture strain of $225 \%$, and UTS of $0.45 \mathrm{MPa}$.

In addition to shape memory properties, PGD displays many beneficial characteristics desirable for future in vivo biomaterial applications. In order to understand the in vivo applicability of PGD, the in vitro degradation rates were explored by Migneco et al in 2009 [19]. By soaking polyester samples in PBS at $37^{\circ} \mathrm{C}$ for 20,40 , and 90 days, the mass loss due to hydrolysis was calculated. A negligible change in mass was observed after 20 days; only $6 \%$ mass loss occurred after 40 days. Over the course of the entire study (90 days), a total of $13 \%$ mass loss was noted. For tissue engineering applications, which need a long time for natural tissue to populate on a PGD device, these results are considered ideal. For other application such as drug delivery, the hydrolytic lifetime of PGD can be shortened by varying the compositions and reaction conditions 
As previously mentioned, glycerol is naturally occurring, with roles in lipid biosynthesis, gluconeogenesis, and glycolysis. Dodecanedioic acid is also endogenous to human metabolism, resulting from the $\omega$-oxidation of lauric acid. Cytotoxicity of PGD was therefore expected to be minimal, as these monomers are compatible with biological systems. In order to explore this hypothesis, human aortic fibroblasts (HAFs) were seeded on PGD-coated glass slides and polystyrene plates [19]. The cell density on the polystyrene plates was higher than that of cells on PGD-coated slides for approximately 18 days. However, at that time point, the cell density on PGD surpassed the density of HAFs on polystyrene. Cell viability and growth were monitored with the WST-1 assay. HAFs were viable throughout the course of the study, with similar trends on PGD and polystyrene [19].

\subsubsection{Fabrication methods}

When fabricating scaffolds for tissue engineering, three methods are commonly used: phase separation, self-assembly and electrospinning [11]. Phase separation fabricates porous polymer membranes and scaffolds by inducing the separation of a polymer solution into a polymer-rich phase and a polymer-poor phase [23]. Self-assembly creates nanofibers that rely on weak noncovalent interactions to build nanofibers from small molecules, nucleic acids, proteins or peptides [24]. Electrospinning is time- and cost-efficient technique that produces long, continuous fibers based on the electrostatic repulsion of a polymer solution to form fibers [12]. Table 1.1 shows the advantages and disadvantages of each method. 
Table 1.1 Three different scaffold fabrication methods and their advantages/disadvantages.

\begin{tabular}{|l|l|l|}
\hline \multicolumn{1}{|c|}{$\begin{array}{c}\text { Fabrication } \\
\text { methods }\end{array}$} & \multicolumn{1}{|c|}{ Advantages } & \multicolumn{1}{|c|}{ Disadvantages } \\
\hline $\begin{array}{l}\text { Phase } \\
\text { separation }\end{array}$ & $\begin{array}{l}\text { Good for creating porous scaffolds. } \\
\text { Simple, no special equipment } \\
\text { necessary. Porosity is easily } \\
\text { controlled. }\end{array}$ & $\begin{array}{l}\text { No organized pattern } \\
\text { possible; limited } \\
\text { number of polymers. }\end{array}$ \\
\hline Self-assembly & $\begin{array}{l}\text { Molecules undergo spontaneous self- } \\
\text { assembly into higher order structures. }\end{array}$ & $\begin{array}{l}\text { Requires engineering } \\
\text { of molecules that will } \\
\text { self-assemble; can } \\
\text { only create short } \\
\text { fibers. }\end{array}$ \\
\hline Electrospinning & $\begin{array}{l}\text { Can create aligned fibrous meshes. } \\
\text { Can be used with biological polymers } \\
\text { such as collagen. }\end{array}$ & Can only create fibers. \\
\hline
\end{tabular}

The main mechanism of phase separation, as the name suggests, is to separate the phases due to their physical incompatibility. One of the phases, which is the solvent, is then extracted, leaving behind the other remaining phases [23]. It is simple and good for creating porous scaffolds, but pattern organization cannot be controlled. For neural tissue engineering, organized patterns are desirable, as they can better guide neural axon elongation. As to self-assembly, the fabrication process is challenging, limited to a few polymers, and can only create short fibers with lengths of one to several micrometer [24]. Comparing these three methods, for neural tissue engineering, electrospinning where the pattern can be controlled, and the fiber length can be adjusted to a few centimeters, is an appropriate choice.

The basic principle of electrospinning involves a Taylor cone of solution, which is held at the orifice of a needle by applying high voltage between the tip 
and the grounded collector. When the electrostatic repulsion in the solution overcomes the surface tension, a charged fluid jet is ejected out of the needle tip, traveling through the air with solvent evaporation, and finally deposited on the grounded collector. The syringe pump provides a continuous flow of the solution coming out of the spinneret and thus multiple copies of the electrospun fibers can be fabricated within a short period of time. During the course of leaving the spinneret and arriving at the collector, the charged jet undergoes stretching and whipping according to a number of parameters, which are broadly classified into solution parameters, process parameters and ambient parameters.

The solution parameters of electrospinning include molecular weight, viscosity, concentration, conductivity, and surface tension. The molecular weight of a polymer plays an important role in determining the size and morphology of electrospun fibers. Generally, high molecular weight polymer solutions are more widely used in electrospinning as they come with the desired viscosity for the fiber fabrication. Studies have shown that a polymeric solution with low molecular weight tends to form beads or beaded fibers rather than smooth fibers [25]. In contrast, a solution with a high molecular weight would yield fibers with larger average diameters. Gupta et al. have utilized PMMA with various molecular weight to investigate the effect of molecular weight of a polymer on fiber morphology. They found that as the molecular weight increases, the number of beads decreases [26]. However, McKee et al. found that high molecular weight is not always essential for electrospinning, as long as sufficient intermolecular interactions can provide a substitute for the interchain connectivity obtained 
through chain entanglements. Nonwoven membranes of oligomer-sized phospholipids, for example, were produced from lecithin solutions via electrospinning [27].

Viscosity of the electrospinning solution also has a significant effect on the morphology and size of the electrospun fibers. No continuous fiber can form if viscosity is too low. On the other hand, ejection of jets from a polymer solution with high viscosity is very difficult. Therefore, an optimal solution viscosity has to be determined in order to fabricate desirable fibers via electrospinning. Fong et al. used polyethylene oxide (PEO) to investigate the effect of viscosity on fiber production, and reported that viscosities ranging from 1 to 20 poise were suitable for uniform nanofiber formation [28].

Polymer concentration is also critical to the production of fibers using the electrospinning process. At a low polymer concentration, the process produces beads, instead of fibers. As the concentration increases, the shape of the beads changes from a spherical form to a spindle-like form and eventually the output becomes uniform fibers. If the concentration is too high, the formation of continuous fibers will be inhibited as it is impossible to maintain the flow of the solution at the tip of the needle. It should be noted that molecular weight of polymer, polymer concentration and viscosity are inter-related to each other. The polymer concentration is strongly related to the viscosity and this relationship has been studied with various polymers, including gelatin, poly (ethylene oxide) (PEO), poly (vinyl alcohol) (PVA), poly (methylmethacrylate) (PMMA), poly (Llactic acid) (PLLA), etc. [26, 29-32]. Normally, increasing polymer concentration 
or viscosity gives rise to a larger or more uniform fiber size. In the electrospinning process, polymer concentration plays an essential role in determining the range of viscosities from which continuous electrospun fibers can be produced. Taken together, previous studies show that there are optimal polymer concentration values for electrospinning and that this property has a remarkable influence on the morphology and diameters of polymeric fibers [33].

The conductivity of an electrospinning solution is typically determined by the type of polymer, the solvent, and the availability of ionisable salts. Previous studies show that there is a marked decrease in electrospun fiber size with the increase of the solution's electrical conductivity, and that with low conductivity, uniform fibers are not produced and beads may also be observed due to the insufficient elongation of the jet by electrical force [34]. Fibers with the smallest diameter can be produced with the highest electrical conductivity polymer and the decrease in the electrospun fiber diameter has been found to be due to increased electrical conductivity [34]. The morphology and diameter of electrospun fibers can be modified by adding ionic salts such as $\mathrm{KH}_{2} \mathrm{PO}_{4}$, $\mathrm{NaH}_{2} \mathrm{PO}_{4}$, and $\mathrm{NaCl}$, e.g. beadless fibers with smaller sizes ranging from 200 to $1000 \mathrm{~nm}$ were fabricated [35]. The same success has also been observed in other polymer types such as polyamide-6 [36], PVA [37], polyacrylic acid [38], PEO [28]. With addition of salts, the uniformity of electrospun fibers increases and bead generation decreases.

Generally, reducing surface tension of the polymer solution leads to the production of beadless fibers in an electrospinning process. Hohman et al. found 
that high surface tension inhibits the electrospinning process due to the instability of the jets and the generation of sprayed droplets [39]. A lower solution surface tension aids the electrospinning process in performing smoothly at lower electric fields; however, a lower surface tension is not always suitable for electrospinning [40]. The surface tension basically determines the lower and upper boundaries of the electrospinning window when all other parameters are constant [28, 37, 41].

The processing parameters of electrospinning include the applied voltage, the delivery rate, the distance from needle tip to collector and the collector types. There is always a threshold voltage in the electrospinning process to ensure fiber formation. When the applied voltage is above this threshold, a sufficient charge is induced on the solution and the electrospinning process is initiated. There is a dispute about the effect of the applied voltage on fiber diameter during electrospinning. Demir et al. claimed that by applying higher voltages, more polymer solution is ejected out of the needle and hence a larger diameter fiber is formed [42]. Other researchers suggested that increasing the electrostatic repulsive force by the increasing applied voltage will narrow the fiber diameters because it forces greater stretching of the solution in the fluid jet [43-45]. Another research group, Reneker and Chun reported there is no significant effect of the applied voltage on fiber diameter with electrospinning of PEO [46]. Therefore, the level of the applied voltage may influence fiber diameter, but its effect is modulated by other parameters such as polymer concentration, solution viscosity, and the tip-collector distance. 
In the electrospinning process, a lower delivery rate is always desirable, as it leads to more time for solvent evaporation. However a minimum delivery rate of the spinning still has to be maintained in order to produce continuous fibers. Electrospun fiber diameter and pore size (determined by fiber diameter) increase as polymer solution delivery rate increases. If the delivery rate is too high, beads tend to form due to the lack of sufficient evaporation time prior to reaching the collector [47-50].

One crucial element of the electrospinning process is the collector type. It serves as the conductive substrate where the electrospun fibers are deposited. Nowadays, many types of collectors, such as rotating mandrel, are used in the electrospinning process [51-56].

Another parameter to control fiber morphology and diameter is the distance between the needle tip and the collector; a minimum distance is required to provide sufficient time for solvent evaporation and produce fibers. It is also observed that a short distance between the needle tip and the collector leads to the formation of flat, instead of round, fibers [43]. However, the effect of this distance on the fiber size and morphology is not as strong as other parameters.

In addition to the solution and the processing parameters, ambient parameters such as humidity and temperature also affect the output of an electrospinning process. Casper et al investigated the effects of humidity on producing polystyrene fibers via electrospinning, and found that increasing the humidity resulted in the appearance of small circular pores on the fiber mat and 
further increase in humidity caused the pores to coalesce [57]. At very high humidity, the discharge of electrospun fibers is promoted. While at very low humidity, the solvent may dry too fast, and the electrospinning process is inhibited [58, 59]. The effect of ambient temperature on electrospinning polyamide- 6 has been studied by Mit-uppatham et al and the results suggest that an increase in the temperature leads to a decrease in fiber diameter. Studies also found that there is an inverse relationship between the temperature and polymer solution viscosity [36].

Electrospinning constitutes a unique technique for the fabrication of micro to nanometer fibers. The advantages associated with fiber scaffolds fabricated by electrospinning include time- and cost- efficiency, high aspect ratio, high specific surface area, and high porosity with a small pore size $[12,60,61]$. A variety of natural and synthetic materials such as gelatin [62], collagen [25], poly( $\varepsilon$ caprolactone) (PCL) [41], poly (D,L-lactic acid- co- glycolic acid) (PLGA) [15, 16], and PLLA [14] fibers have been successfully produced using the electrospinning process. These electrospun fibers will play a significant role in different tissue engineering areas and typical examples of which include neural, bone, cartilage, skin, tendon and ligament and cardiovascular tissue engineering [63-68].

\subsubsection{Stem cells and its differentiated neurons}

In the neural system, neuron or glial cell degeneration could lead to various clinical disorders. Nerve injury is a daunting challenge because most of these injuries result in irreversible loss of function. Therefore, another key element of neural tissue engineering is employing normal or genetically 
engineered cells to repair injured locations. Different types of cells such as embryonic stem cells, induced pluripotent stem cells and neural stem cells have already been studied by researchers.

\subsubsection{Teratomas, pluripotent stem cells, and mouse embryonic stem cells}

Teratomas, a rare clinical tumor, is the beginning of the whole pluripotency story. It carried all three germ layers: ectoderm, mesoderm and endoderm, similar to what is found in a developing embryo [69]. Thanks to Stevens study [70] on spontaneous testicular teratomas and later the work of Kleinsmith [71] and Pierce on teratocarcinoma, the embryonal carcinoma (EC) cell lines were developed and the mouse embryonic stem cells (mESCs) were finally established from the inner cell mass of mouse blastocysts in 1981 by two different groups[72, 73]. Initially, mESCs were cultured in conditioned medium of teratocarcinoma and feeder layer cells. Later on, Leukemia Inhibitory Factor (LIF) was identified and purified for maintaining the pluripotency of mESCs [74]. From then on, mESCs routinely maintained in labs became the working horse to help explore the unknown stem cell and developmental biology.

1.2.2.2 Human embryonic stem cells

Although the use of human EC cell lines in research dates back to 1970's [75], the equivalent human embryonic stem cells (hESCs) was not established until 1998 by Thomson et al. [76]. The explanation of this technical delay in hESCs could be due to the different requirements of maintaining hESCs. Unlike the mESCs whose self-renewal is dependent on the LIF activated STAT3 
signaling pathway [77], undifferentiated hESCs are maintained in the presence of mouse embryonic fibroblast feeders [76]. For the sake of maintaining hESCs in a feeder-free condition, condition medium from feeder cells plus Fibroblast growth factor-2 (FGF2) [78], high concentration FGF2 [79], or Transforming growth factor-beta (TGF- $\beta$ ), Nodal and Activin A $[80,81]$ had been used for supporting undifferentiated culture on Matrigel or other ECM coating plates.

Furthermore, there are several differences between mouse and human embryonic stem cells, such as colony morphology and surface markers (SSEA1 vs. SSEA3), even though they both share the same core regulatory transcription factors like Oct4, Sox2 and Nanog for pluripotency. Recent studies indicated that mouse and human embryonic stem cells may represent different developmental stages of pluripotent stem cells. Especially hESCs are more similar to mouse epiblast stem cells in terms of morphology, growth requirement and gene expression profiles $[67,82]$.

Since the successful culturing of hESCs, those cells attracted the attention of the scientific community to explore their potential clinical applications based on investigating basic stem cell biology. The unique self-renewal and pluripotent properties of hESCs provide an unlimited cell source for tissue engineering and regenerative medicine.

To date, the utilization of human embryos to create hESCs lines still presents ethical and political concerns, resulting in fervent debate regarding this area of research. Another concern for ES cell transplantation is the genetic differences between the ES cells and the patient; immunosuppression drugs may 
be required to prevent rejection [83]. In order to develop patient- specific pluripotent stem cell, an alternative strategy called Somatic Cell Nuclear Transfer (SCNT), based on the early discovery of Goudorn's study by replacing an oocyte's nucleus with another somatic cell's nucleus [84], had been proposed and demonstrated in mammals [85-87]. But this approach also faces the same ethical considerations for using human oocytes and destroying resulted embryos as well as technical challenges [88].

\subsubsection{Induced pluripotent stem cells}

A recent exciting development of mouse induced pluripotent stem cells (iPSCs) by Takahashi et al. [89] opened a new road for converting somatic cells to pluripotent stem cells. They carefully screened two dozens transcription factors that are highly expressed in ES cells and finally narrowed down to four key factors that are sufficient for reprogramming. By transducing four transcription factors (Oct4, Sox2, Klf4, and c-myc), also called "Yamanaka factors", into mouse embryonic or adult fibroblasts, these reprogrammed somatic cells exhibited mouse embryonic stem (ES) cell-like properties in terms of morphology, growth properties and gene expression patterns. In addition, they can form teratomas after injection into mice. After a few months, Wernig et al. [90] reached the same conclusion for mouse iPSCs with ES-like properties, and further showed that mouse iPSCs could form chimaeras as well as generate functional germ cells.

In less than a year, Yamanaka's group [91] demonstrated again that the same four transcription factors could be used to obtain human iPSCs. 
Immediately, other groups confirmed this somatic cellular reprogramming feasibility [92-94] and demonstrated that other defined factors (Oct4, Sox2, Nanog, and LIN28) also worked [92]. It was concluded that human iPSCs and human embryonic stem (ES) cells are similar in regards to their morphology, growth, surface antigens, gene expression, and telomerase activity. Furthermore, these cells could differentiate into cell types of the three germ layers in vitro and form teratomas in vivo.

Even though the efficiency of reprogramming iPSCs is still low and it exhibits some safety concerns of using viruses for delivery of exogenous genes and having the potential to form teratomas from undifferentiated cells, this technique still attracts a lot of scientific interest. Due to the simplicity and repeatability of iPSCs technology, multiple somatic cells and species have been tested and validated. The advancement of this technique resulted in explosive research activities in the past few years and is expected to accelerate the development of the use of iPSCs from patients for therapeutic purpose. One of the biggest advantage of using iPSCs is that the original source cells can be derived directly from patients.

\subsubsection{Neural stem cells, neurons and glial cells}

Due to limited access to human neural tissues, direct harvest of differentiated neural cell or adult neural stem cells is not always a viable choice. The characteristic properties of pluripotent stem cells are the capability of selfrenewal and differentiation into any cell type of three germ layers. Since the establishment of hESCs, a great deal of research have been made to explore the 
potential of differentiating hESCs to clinically relevant cell population such as dopaminergic neurons for Parkinson's diseases and motor neurons and/or oligodendrocytes for spinal cord injuries.

Interesting observations of the pluripotent stem cell differentiation process seemed to recapitulate the major events in the embryo development. Thus carefully following the sequences of embryo development and providing the right combination of spatial and temporal signals normally led to specific lineage cells [95]. For the differentiation of pluripotent stem cells to neuroectoderm lineages, some studies have used conditioned media from stromal cells [96, 97], or induced the formation of $3 \mathrm{D}$ embryoid bodies. The early study results had indicated that in the absence of BMP signals, pluripotent stem cells would differentiate into neuroectoderm lineage in default [98]. Therefore, a chemicallydefined medium will help scientists identify the mechanisms with less interferences and accelerate the potential clinical application [99].

Multipotent neural stem cells (NSCs) or neural progenitor cells (NPCs) are capable to differentiate into three major cell types: neurons, astrocytes, and oligodendrocytes. For research purposes or clinical applications, more specific subtypes of neurons and glial cells might be needed. For example, Wichterle et al induced the differentiation of motor neurons from ES cells derived NSCs by using retinoic acid and sonic hedgehog (SHH) analog [100]. Another clinically relevant dopamine neurons for Parkinson's disease had been shown to differentiate from mouse [101] and human [102, 103] ES-cell derived NPCs in the presence of FGF-8 and SHH. In addition, Nistor et al. [104] demonstrated the 
step-wise differentiation procedures for producing oligodendrocytes from human ES-cell derived oligodendrocyte progenitors.

\subsubsection{Motor neuron}

Motor neurons originate in the motor region of the cerebral cortex, have cell bodies in the spinal cord and axons that either directly or indirectly extend to control muscles. It is clear that these cells have the potential to be very long, especially when providing impulses to muscles in distal regions. In all vertebrates, motor neurons are cholinergic, thus releasing only the neurotransmitter acetylcholine, and a single motor neuron has the potential to innervate numerous muscles simultaneously. In vertebrates, motor neurons can only provide an excitatory, contractile signal, and thus relaxation can only occur by inhibiting the motor neuron itself.

Motor neuron diseases are characterized as primarily affecting either the upper motor neurons or lower motor neurons [105]. Most often, motor neuron disease has a genetic origin, with the primary associated diseases being amyotrophic lateral sclerosis (ALS), spinal muscular atrophy (SMA) and bulbospinal muscular atrophy. While in recent years there has been significant progress in identification, classification and pathogenesis of motor neuron diseases, there are still no available treatments for patients suffering from these degenerative pathologies. Direct implantation of motor neurons derived from embryonic lines or induced pluripotent lines is still in the realm of science fiction, although the protocols are slowly improving. Alternatively, deriving a pharmacological solution will require extensive testing on cultured motor neurons 
or animal models. This pharmacological, cell-based approach is far more clinically relevant at this time [106].

Current neural induction protocols typically require protein introduction, conditioned media, feeder layers or SMAD signaling inhibition. Each of these techniques has a significant drawback, such as poorly defined culture conditions, elongated time-course or low yield.

The most typical motor neuron differentiation protocol requires the introduction of a protein called Sonic Hedgehog $(\mathrm{SHH})$ in the presence of Retinoic Acid (RA). The starting step of this protocol requires the creation of neuroepithelial cells from either ESCs or iPSC lines [107-109]. These neuroepithelial cells form tube-like rosettes in human cell lines over the first two weeks of culturing. In mouse lines, these rosettes do not form. The addition of $\mathrm{SHH}$ in the presence of RA induces the cells to form motor neuron progenitors which express OLIG2. It has been recently found that the protein $\mathrm{SHH}$ can be replaced with the far less expensive small molecule PUR, with similar effects [110]. One week later, these progenitor cells develop into immature, HB9expressing motor neurons. After another three to five weeks, functional mature human motor neurons can be identified.

Instead of using this protein induction approach, the use of conditioned media has been identified as a viable alternative. With this approach, it is necessary to culture the embryoid bodies in a conditioned media derived from the human hepatocarcinoma cell line HepG2 (MedII) [111]. Using this conditioned media at the embryoid body stage, neural precursors quickly emerged. While 
this approach is more complicated than the protein induction approach, due to the need for conditioned media from a carcinoma cell line, it demonstrates the novel possibility of using media conditioning as a technique for neural induction. Unfortunately, this technique does not currently allow the specification of the type of neuron derived unlike the more targeted protein induction approach.

Combining the approaches of protein induction and cellular conditioning forms the stromal feeder approach. In this approach, embryoid bodies are first cultured on MS5 stromal feeders [112]. These feeder layers promote a quick and efficient generation of neural precursors, which following the introduction of $\mathrm{SHH}$ and RA creates spinal motor neurons. The feeder layer approach has been proven to be effective in generating not only neural cells but also a wide range of glial cells. While it is slightly quicker than the protein induction method on its own, its cost is significantly higher.

The final technique for discussion is the use of SMAD signaling inhibition. SMAD signaling plays a tremendous role during neural induction and recent studies have shown that particular elements of this signaling cascade, such as bone morphogenic protein (BMP) inhibitors, are critical during the neural organization phase of embryonic development [113]. This particular protocol utilizes the same steps as the protein induction explanation above, but also adds two additional molecules - inhibitors of SMAD signaling called Noggin and SB431542. This protocol has been demonstrated to be robust and effective, as it generated a complete neural conversion of over $80 \%$ of human ESCs in adherent culture conditions. 
1.3 Current research of cellular interactions on electrospun scaffolds for neural tissue engineering

Central or peripheral nerve injury which cannot be directly repaired by end-to-end sutures is still a daunting challenge in clinical research. Nerve autograft transfers are the current gold standard for treating large nerve gaps but limited by shortage of donor nerve, donor site morbidity and inadequate functional recovery. A potential regenerative strategy is stem cell transplantation; however, less than $1 \%$ cell survivability is far from the minimal requirement $[9$, 114]. To improve cell viability, stem cells can be transported via an artificial scaffold, which provides an environment conducive to survival after delivery. Electrospun nanofibers are well suited for this application as they can not only promote the neural cell differentiation but also enhance the axonal elongation.

Neural cell proliferation and differentiation were shown to be affected by several properties of nanofiber scaffolds. Yang et al. studied the influence of fiber alignment on the differentiation of neural stem cells (NSCs) [14]. They fabricated aligned nanofibers by using a rotating disc as a collector. The results demonstrated that NSCs elongated and their neuritis outgrew along the orientated nanofibers. In another study, Xie et al. reported that aligned PCL nanofibers not only enhanced the differentiation of embryonic stem cells into neural lineage cells but also controlled the direction of neurite extension [115]. In addition, the diameter of fibers has been shown to affect NSCs differentiation and proliferation. Christopherson et al. claimed that as the diameter of fiber decreased, the NSCs showed more migration, spreading and proliferation [63]. 
Furthermore, it was observed that the NSCs showed an increased rate of differentiation and proliferation on oriented nanofibers compared with microfibers [14].

Some recent studies have proved that the conductivity of nanofibers can also influence the neural cell behavior. Ghasemi-Mobarakeh et al. obtained conductive electrospun nanofibers by mixing polyaniline (PANI) with poly ( $\varepsilon$ caprolactone)/ gelatin (PG). Electrical stimulation through 15\%PANI/85\%PG nanofibrous scaffolds showed enhanced NSCs proliferation and neurite outgrowth [116]. Cullen et al. fabricated nanofibers consisting of a blend of PANI and polypropylene as living neural-electrical relays. This fiber scaffold shows the potential to promote primary dorsal root ganglion neurons adhesion, growth and neurite extension [117]. In another work, Liu et al. used Polypyrrole (PPy) substrates doped with chondroitin sulfate (CS) and conjugated with type I collagen. The collagen could self-assemble into 3-dimensional fibrillar matrix at the polymer surface. Their results showed that rat pheochromocytoma cells had an increased differentiation rate on the polymer substrates and electrical stimulation promoted the neurite outgrowth [118].

Other research groups have worked on incorporating proteins onto electrospun nanofiber scaffolds. Liu et al. investigated the feasibility of loading chondroitinase $A B C$ (ChANS) and neurotrophin-3(NT-3) onto electrospun collagen nanofibers [119]. By using microbial transglutaminase (mTG) mediated crosslinking, the protein incorporating efficiency could reach $45-48 \%$. Their results demonstrated that sustained release of NT-3 and ChABC through 
collagen nanofibers can promote axonal regeneration and thus could be useful in spinal cord injuries treatment. Further, another research group immobilized EGF and bFGF onto PLLA nanofiber scaffolds and found significant enhancement of the axon growth [120]. Lee et al. coated PLGA nanofibers with PPy and immobilized nerve growth factor (NGF) on to the surface. These NGFimmobilized PPy-coated PLGA scaffolds showed the supportive of PC12 neurite formation and outgrowth [121].

\subsection{Research Plan}

The objective of this study is that electrospun fiber scaffolds made from PGD and its modified derivative are able to support ESCs proliferation and differentiation into functional motor neurons using the newly developed protocol.

To complete the objective of this $\mathrm{PhD}$ research, several experimental studies (specific aim 1, 2 and 3) were designed and sequentially carried out. These studies reflect the progression of the development this $\mathrm{PhD}$ research, and hence become the frame work of this dissertation.

In Chapter 2 of this dissertation (Specific aim 1), the development of an efficient and economical approach to direct the differentiation of ESCs to motor neurons is presented. The reason behind this development is that a large number of motor neurons is required to carry out a research project that aims to develop a new therapy for nerve injuries (i.e., the scope of this $\mathrm{PhD}$ research). Primary culture of motor neurons is not a feasible option here because the survival and proliferation of primary motor neurons tend to limited in an in vitro environment. 
The use of ESCs is considered because they could, potentially, proliferate unlimitedly and can be derived into neurons as well as other neural cell types using different protocols. To successfully realize this idea, a small-molecule based methodology to differentiate ESCs into defined neural lineages in a fully controlled and efficient manner was developed. This protocol was tested with ESCs on the culture dishes as well as the electrospun fibers.

In Chapter 3 (Specific aim 2), the focus will shift to scaffold building. Electrospinning is employed in this $\mathrm{PhD}$ research to fabricate fibers from polymer PGD, a new class of biodegradable and biocompatible polymers developed by Migneco et al in 2009 [19], and its gelatin blended long fibers. The morphology, chemical structure, dissolvability and biocompatibility of the electrospun fibers as well as the effects of fabrication parameters on these characteristics are reported in this chapter.

However, one disadvantage of the PGD polymer is that it is composed of saturated polyester chains that only have hydroxyl or carboxylic groups available for chemical modification. If another double-bond containing monomer is introduced, the resulting polymers with unsaturated polyester chains may provide new modification possibilities. These properties, in turn, can be used to modify PGD fibers and facilitate cell attachments. For this reason, fumaric acid, a nontoxic, naturally occurring monomer, was incorporated into the PGD polymer synthesis process and produced a PGD derivative referred as poly (glycerol dodecanedioate co-fumarate) (PGDF) (Specific aim 3). It was combined with gelatin and PEO to fabricate electrospun fiber scaffolds. Both PGD and PGDF 
based scaffolds were then used in a study of the growth and morphology of neural lineage cells to determine the effects of newly fabricated scaffolds on cell proliferation and differentiation. The results of this study are presented in Chapter 4 of this dissertation.

In the final chapter of this dissertation, a summary of the scientific findings of this Ph.D. research is provided, which supports the research hypothesis and demonstrates the success of this research project. In addition, a critical evaluation of the potential of this research project as well as a list of future work required to realize the ultimate goal of this research (i.e., developing a new therapy for nerve injuries) will be provided. 
CHAPTER 2 Efficient and Low-Cost Differentiation of Embryonic Stem Cells into Motor Neurons by Metabolites for Neural Tissue Engineering

\subsection{Introduction}

As mentioned in chapter 1, efficient cell differentiation protocol is one of the important factors for neural tissue engineering. Other than the typical protocols for neuron differentiation mentioned previously, small molecule techniques have emerged in recent years to provide an inexpensive means of differentiating pluripotent or embryonic lines into motor neurons. This is accomplished most often by inhibiting or stimulating particular pathways that guide the differentiating cells into their final desired form. Small molecules that have been used so far in recent literature in the generation of neurons include Dorsomorphin (DM), SB431542 (SB), CHIR99021 (CHIR), and Purmorphamine (PUR) [122].

DM is an inhibitor of AMP-activated protein kinase (AMPK) and BMP signaling. It is believed that DM assists neural induction by inhibiting BMP Type1 receptors ALK2, ALK3 and ALK6 [123]. In addition to the BMP-inhibition of DM, it has been recognized that SB must also be added due to its effect of inhibiting TGF- $\beta$. SB has been shown to specifically inhibit TGF- $\beta$ type 1 receptors, also known as activin receptor-like kinase 5 (ALK5) [124] while having no effect on BMP signaling. The combination of these two small molecules allows initial neural induction.

Neural induction is also maintained by inhibition of the Wnt- $\beta$ pathway, carried out by using the small molecule CHIR. The Wnt- $\beta$ pathway has been 
implicated in neural crest induction but only recently has it been demonstrated that by inhibiting the pathway the ectoderm is able to expand the size of the neural plate [125]. When upregulated during this developmental phase, neural plate formation is not properly induced. Thus, it is clear that this pathway is another potential target for low-cost neural induction.

The final small molecule for discussion is PUR, which is currently used in recent literature (as well as in this particular study) as a replacement for the more expensive $\mathrm{SHH}$. $\mathrm{SHH}$ is a well-studied morphogen and has been shown to contribute to floor-plate development within the neural tube. Specifically, SHH has been shown to induce the differentiation of motor neurons and ventral interneurons [126]. Fortunately, PUR fulfills all of these same functions, and thus the addition of PUR is a crucial step when inducing neural cells towards a motor neuron lineage [110].

While the previously described small-molecule techniques improve induction and differentiation efficiency, the metabolite techniques utilized in this study were mostly used to improve survival. Specifically, Sodium Fumarate (NaF) and Genistein were applied to reduce oxidative stress and Sodium Butyrate (NaB), a histone deacetylase (HDAC) inhibitor, to promote neural cell differentiation. The use of these metabolites was both individually and collectively tested.

$\mathrm{NaF}$ has been demonstrated to be both neuroprotective and cardioprotective through activation of the Nrf2 antioxidant pathway [127, 128]. $\mathrm{NaF}$ is derived from Fumaric Acid, which is a metabolite and intermediate 
product of the citric acid cycle [129]. Time-dependent and concentrationdependent studies have been carried out that demonstrate $\mathrm{NaF}$ to be immunomodulatory and able to reduce oxidative stress without altering fundamental network activity $[127,128]$. In vitro, its addition has been shown to improve neuronal survival. In vivo, NaF works to better preserve the integrity of myelin, axons and neurons [129]. The choice to add it to this experimental protocol was due to a fundamental belief that its preservative ability would impact cell survival and induction efficiency.

Genistein was chosen for a very similar reason, the reduction of oxidative stress, although it also aids in neural induction. Genistein is an isoflavone, a small molecule that has estrogen-like activity, and is believed to be useful as a preventative agent for hormone-related cancers [130]. Genistein is a secondary metabolite originating from plants, although its specific mechanism of action is unknown. It is also an inhibitor for protein phosphorylation at tyrosine residues, a process that researchers believe is responsible for neural differentiation [131]. In addition to its inductive ability, it has been proven to aid in neuronal cell viability and proliferation in vitro [132]. Genistein's ability to modulate and reduce mitochondrial oxidative stress reduces neural apoptosis, even further supporting survival [133]. Similarly to NaF, Genistein was tested in this protocol due to its inductive and protective abilities.

$\mathrm{NaB}$ was chosen for this study due to its role as an HDAC inhibitor and a metabolite. HDACs have been experimentally demonstrated to compact chromatin and silence genes, and are implicated in numerous dysfunctional 
neuronal conditions, such as depression, addiction and memory problems [134] [26]. This same study examined the effects of inhibitions of different HDACs on mouse embryonic neurogenesis and demonstrated that the inhibition of HDACs by $\mathrm{NaB}$ inhibit $\mathrm{BMP}$ signaling and promote neurogenesis. Thus, $\mathrm{NaB}$ acts similarly to the small molecule Dorsomorphin, referenced above. $\mathrm{NaB}$ is a metabolite, however, and thus is more useful for controlling which metabolic pathways are activated. While $\mathrm{NaB}$ has been studied generally in neurons, it has not been studied specifically concerning its impact on the survival and differentiation of motor neurons. HDAC inhibitors have also been demonstrated to promote differentiation of embryonic stem cells into neural cells in adherent monoculture, but in a surprising reversal, they have also been demonstrated to assist lineage-committed oligodendrocyte precursor cells in converting into multipotent cells akin to neural stem cells $[135,136]$. Due to its aid in inducting embryonic stem cells into neural lines, NaB was utilized in this study.

These metabolites were essential to the study because of their roles as metabolites. Each of the three just mentioned, as well as RA, are important intermediates or byproducts of metabolic mechanisms, and are thus important for upregulating or downregulating particular metabolic pathways. RA is the active metabolite of Vitamin A and a powerful regulator of gene transcription [137]. Vitamin A was chosen due to its effects on cell growth and immune function. $\mathrm{NaF}$, the intermediate of the tricarboxylic acid (TCA) and Kreb Cycle, was selected since glycolytic metabolic mechanisms inhibit stem cell induction. While NaB's role as a metabolite is poorly understood, it has been demonstrated to 
regulate acetyl-CoA synthesis [138]. Genistein's metabolism mechanism is also not well documented, however its status as a metabolite is confirmed. While the particular mechanisms by which these metabolites are used are poorly understood, they all have been individually linked to the downregulation of glycolysis and upregulation of oxidative phosphorylation that is typical to induction mechanisms. By using metabolites instead of other typical small molecules such as cytokines or growth factors, there is a potential to maximize the effect on the cell's metabolic pathway while simultaneously reducing the input cost.

After indicating that each of these small molecules is a known metabolite, now it is the time to demonstrate why metabolic pathway modulation is the key to the motor neuron induction. Stem cells are well known to have different metabolic mechanisms and requirements than differentiated somatic cells. It is believed that stem cells alternate between self-renewal and differentiation through metabolic changes, modifying the mammalian target of rapamycin (mTOR) and AMPK pathways [139]. Since DM is a well-known AMPK inhibitor, metabolic modification may be a key component of its use in aiding neural induction. Accumulating evidence has suggested that metabolites may be essential in modulating the cell signaling pathways and epigenetic regulation, and through using them, it is able to stimulate specific metabolic pathways that favor induction.

Through these modifications, stem cells begin to favor aerobic glycolysis over oxidative phosphorylation. ESCs specifically have a lower oxygen 
consumption rate, less mitochondrial membrane potential and a higher concentration of glycolytic enzymes, thus favoring glycolysis [140]. The aerobic glycolysis cycle is subject to substantial energy demands due to the shortened cell cycle of the stem cells. In order to promote the stem cell differentiation, the energy production pathway was shifted away from glycolysis by using Dulbecco's Modified Eagle's Medium (DMEM) Low Glucose medium, which has been validated in previous literature [108]. The additional metabolites also contributed to the shift away from glycolysis. Following the discussion of the results of the particular metabolite approach, potential metabolic mechanisms that aided motor neuron induction strategy will be addressed and how each metabolite was used to shift energy production towards oxidative phosphorylation will be proved. It is believed that through understanding the metabolic underpinnings of stem cell differentiation, novel metabolite approaches can be easily derived to provide lowcost approaches to stem cell differentiation.

In this chapter, highly efficient and cost-effective production of motor neurons from mESCs through the application of metabolites (RA, Genistein, NaF, and $\mathrm{NaB}$ ) was examined. These metabolites have not collectively been tested specifically with motor neurons until now and only RA has been used at all to induce this cell type.

\subsection{Experimental}

2.2.1 Motor Neuron Induction from Mouse Embryonic Stem cells

Green fluorescent protein (GFP) -Expressing mESCs were purchased from GlobalStem. The procedure for maintenance of mESCs was as follows: The 
mESCs were cultured on poly-L-lysine/laminin (Sigma-Aldrich, Fisher Scientific) coated culture dishes in embryonic stem cell growth medium (GM, DMEM-High glucose supplemented with 15\% Fetal bovine serum (FBS), $10 \mathrm{ng} / \mathrm{ml} \mathrm{LIF}$, and $0.1 \mathrm{mM} \beta$-mercaptoethanol). Before neural induction, RA (1 $\mu \mathrm{M}$, Sigma-Aldrich) was added into the GM for 24 hours (Day0). On day1, cells were cultured in neural induction medium (NIM, 1:1 mix of DMEM-Low glucose and F12 supplemented with B27 (Invitrogen), $1 \mu \mathrm{M}$ Genistein and $0.25 \mathrm{mM}$ Sodium Butyrate (NaB) (All from Sigma-Aldrich) for 3 days. The medium was changed every day. On day4, $1 \mu \mathrm{M}$ RA, $5 \mathrm{ng} / \mathrm{ml} \mathrm{SHH}$ (PeProTech), 200 $\mu \mathrm{M}$ Sodium fumarate $(\mathrm{NaF})$ and $1 \mu \mathrm{M}$ Genistein were added for another 5 days for motor neuron induction. On day $9, \mathrm{RA}, \mathrm{NaF}$, Genistein and PUR/SHH were removed from the medium and cells were cultured with $10 \mathrm{ng} / \mathrm{ml}$ brain-derived neurotrophic factor (BDNF, PeProTech), $10 \mathrm{ng} / \mathrm{ml}$ glial cell-derived neurotrophic factor (GDNF, PeProTech), and $0.5 \mathrm{mM}$ Creatine (Sigma-Aldrich) for 2 days or longer.

2.2.2 Functional evaluation of motor neuron differentiation

2.2.2.1 Reverse Transcription-Polymerase Chain Reaction

Total RNA was prepared from motor neuron differentiation cultures using SV Total RNA Isolation System (Promega). Each mRNA preparation was reverse transcribed into cDNA using olig(dT) $)_{15}$, as described by the manufacturer (GoScript Reverse Transcription System, Promega). Real-time Polymerase Chain Reaction (PCR) was performed on a Real Time PCR System (Applied Biosystems). For amplification, the cycling parameters consist of 1 cycle at $95{ }^{\circ} \mathrm{C}$ 
for $2 \mathrm{~min}$ and $20 \mathrm{~s}$, followed by 40 cycles of $95{ }^{\circ} \mathrm{C}$ for $3 \mathrm{~s}$ and $60{ }^{\circ} \mathrm{C}$ for $1 \mathrm{~min}$. The comparative CT method was utilized to calculate the relative expression of each target gene, and each is normalized relative to the expression of glyceraldehydes-3-phosphate dehydrogenase (GAPDH) mRNA.

\subsubsection{Immunocytochemistry}

Motor neurons were subcultured on laminin-coated $35 \mathrm{~mm}$ dishes and fixed by using SafeFix (SafeFix ${ }^{\mathrm{TM}}$ II All-Purpose Fixative, Fisher HealthCare) at room temperature for $15 \mathrm{~min}$. Immunocytochemical staining was performed using standard protocols and antibodies against MAP2(1:100; GeneTex), ChAT(1:50, GeneTex) and HB9 (1:50, Santa Cruz). Antibodies against $\beta$-Tubulin3 (E7; 1:12.5), and Nestin (Rat-401; 1:5) were purchased from the Developmental Studies Hybridoma Bank. As secondary antibodies, donkey anti-mouse IgG $(H+L)$, donkey anti-goat $\lg G-T R$ and goat $F(a b) 2$ anti-rabbit $\lg G(H+L)$ (Southern Biotech) were used against the primary antibodies. Nuclei were counterstained with 4', 6'-diamidino-2-phenylindole (DAPI, Sigma). Images were collected using a fluorescence microscope equipped with phase contrast optics and camera (Nikon).

\subsubsection{Evaluation of cellular viability}

Resazurin fluorescent dye (Sigma-Aldrich, St Louis, MO), which had been shown to be identical to the commercial assay Alamar Blue, was used as an index of cellular viability [141-144]. Briefly, 1:10 dilution of the reagent was mixed with fresh medium and added to each cell plate. Background controls of 1 $\mathrm{ml}$ of medium plus $100 \mu \mathrm{l}$ of the resazurin reagent were also performed. The 
plates were placed in an incubator at $37{ }^{\circ} \mathrm{C}$ and $5 \% \mathrm{CO}_{2}$ for 4 hours to allow the cells to metabolize the resazurin. Then, $100 \mu \mathrm{l}$ medium samples from different cell plates were collected into a 96-well plate in triplicate. Thereafter, the 96-well plate fluorescence reader was set to measure the fluorescence intensity of a 590 $\mathrm{nm}$ emission with an excitation wavelength of $530 \mathrm{~nm}$. The same procedure was repeated at different time points (day1, day3, day5, day7, day9 and day11). The experiment was repeated three times and each individual group had three separate samples.

\subsubsection{Extracellular Electrophysiology}

For recording electrophysiological properties, a $35 \mathrm{~mm}$ plastic dish containing cultured motor neurons in growth medium was used. Prior to electrophysiology measurements, cells were kept at $37^{\circ} \mathrm{C}$ in a $5 \% \mathrm{CO}_{2}$ incubator. The dish was quickly transferred to the stage of an inverted microscope for study. Cells were recorded within a dish containing the differentiation media. The ground of the pre-amplifier was connected to the dish media and metal microelectrodes were slowly lowered inside. The faraday cage was connected to the ground of the oscilloscope, which was located outside the faraday cage.

To study the electrical characteristics of the motor neurons, extracellular electrical stimulation was used to depolarize the neurons, after which they generated action potentials typical of native tissue. $1 \mathrm{M} \Omega$ impedance metal electrodes (Eppendorf) were used to depolarize small clusters of neurons in the dish. These electrodes were connected to a preamplifier (A-M Systems) ,which was in series connected into an AC/DC Differential Amplifier (A-M Systems 
Model 3000). This amplifier has a gating feature, which allows the metal electrodes to be capable of both recording/amplifying a signal or transmitting electrical stimulation, depending on the voltage level of a trigger input. To control the complex timing of the system, a Native Instruments Data acquisition (DAQ) Board (Native Instruments M-Series) was used. Once the DAQ Board sets the amplifier's trigger into stimulus mode, it triggers a pulse stimulator (A-M Systems Isolated Pulse Stimulator Model 2100) to send a depolarizing pulse through the amplifier and electrodes into the dish. Immediately following the pulse, the DAQ board sets the amplifier into recording mode and records a five-second signal at $50 \mathrm{k} \mathrm{Hz}$. All software to handle the timing was composed and executed in Matlab.

To depolarize the motor neurons, a pulse of length $0.2 \mathrm{~ms}$ was used. To determine the proper voltage/current to depolarize the cells, a low current of 30 $\mu \mathrm{A}$ was used for stimulation and was raised in steps of $10 \mu \mathrm{A}$ until a signal was seen. The amplifier was kept at a constant bandwidth of $300-20 \mathrm{k} \mathrm{Hz}$ and an amplification of 1000 . A notch filter at $60 \mathrm{~Hz}$ was used to eliminate line noise.

\subsection{Results}

2.3.1 Efficient motor neuron induction from embryonic stem cells

\subsubsection{Schematic procedures of motor neuron differentiation}

The procedure for motor neuron induction is schematically summarized in Figure 2.1A. Immediately after plating the mESCs (Figure 2.1B), a 24-hour RA treatment was applied. This novel strategy was adopted to promote the epithelialmesenchymal transition (Figure 2.1C). Following this treatment, a combination of Noggin, Genistein and NaB was applied for 3 days to promote the establishment 
of neural stem cells, as measured by neural stem markers PAX6+/Nestin+. These cells were allowed to continually expand to provide resources for future experiments. For 5 days, these cells were incubated with RA, SHH/PUR, Genistein and $\mathrm{NaF}$ in order to generate motor neuron precursors (Figure 2.1F). Finally, a two-day treatment with BDNF, GDNF and Creatine was applied in order to create functionally mature motor neurons (Figure $2.1 \mathrm{G}$ ). The phase contrast microscopy in Figure 2.1(B-G) demonstrates the morphological maturation of the motor neurons.

A
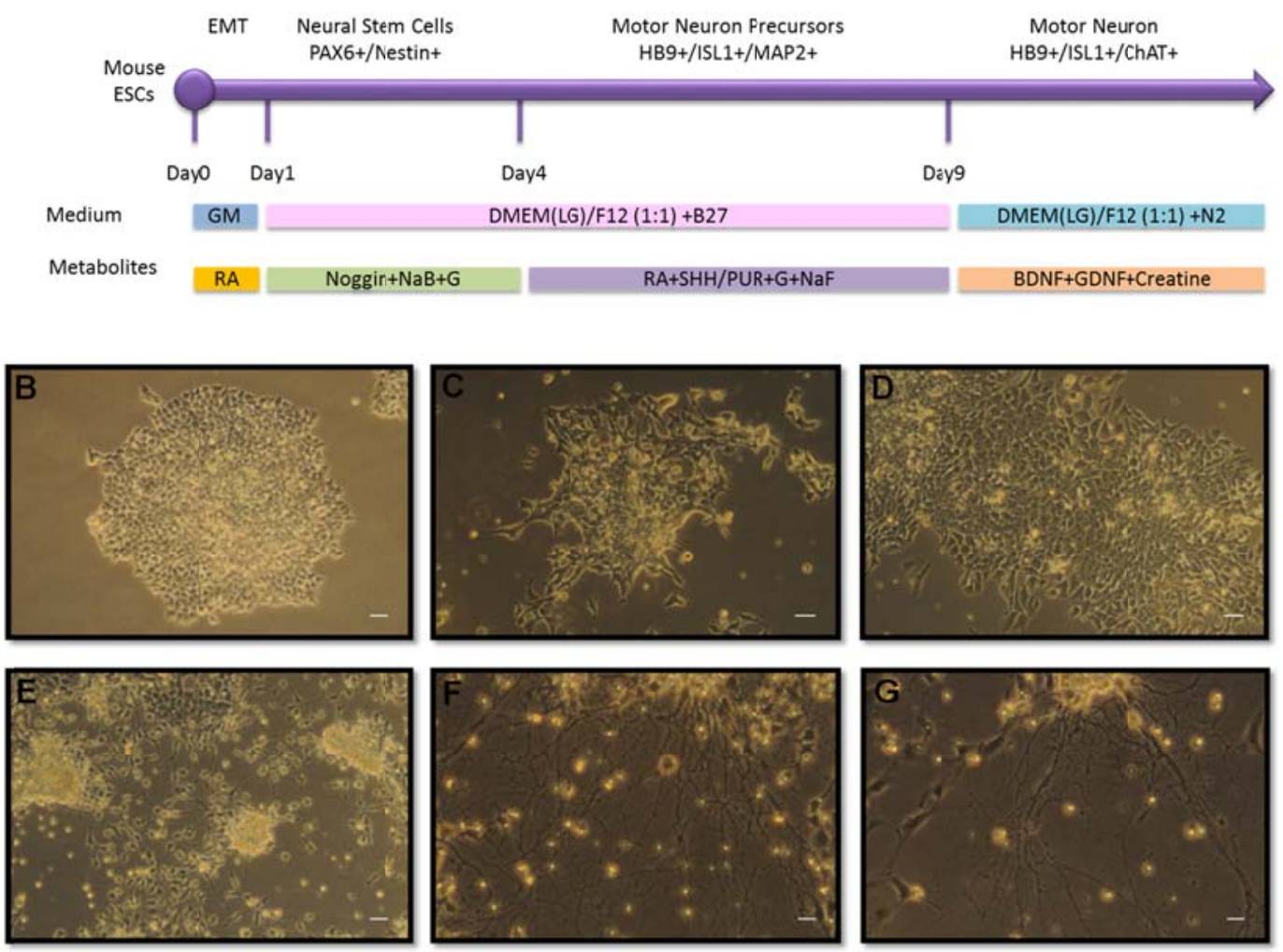

Figure 2.1. Motor neuron differentiation and morphology of mouse embryonic stem cells. (A) Schematic procedures of efficient motor neuron differentiation system from mESCs. (B-G) Sequential images of motor neuron differentiation of mouse embryonic stem cells. (B) Mouse ESCs were cultured on poly-L-lysine/laminin plates. (C) One day after treating with RA. (D) After 3 days in neural induction medium, cells lost their typical embryonic stem cell dome shape and 
became flat. (E, F) Neural stem cells were cultured in the presence of RA and SHH for 2 days and 5 days. (G) Two days of axon elongation promotion. Scale bars: (B-D) $100 \mu \mathrm{m}$; (E-G) $10 \mu \mathrm{m}$.

2.3.1.2 RA Increases the Cell Viability and Restricts ESCs into Neuronal Lineage

The novel use of RA for a 24-hour incubation step on day 0 provided significant improvement in cell viability and transformational efficiency. Figure 2.2A showed that the application of RA treatment reduced the expression of the essential pluripotency factors Oct4 and Sox2 compared to the control group. The difference between the decrease of Oct4 and Sox2 indicated that the expression balance of these two factors was disturbed, which broke the maintenance of the mESCs pluripotent state and leaded cells down the neural ectodermal cell lineage (Figure 2.2B). After RA treatment, the mESCs started to lose typical colony morphology and the cells began to migrate away from the colony. The Immunocytochemistry images in figure $2.2 \mathrm{C}$ clearly showed that the control group has more Oct4+ and Sox2+ cells than the experimental group. However, the high expression of pluripotent stem cell markers implied that most cells are still not differentiated for day $0 \mathrm{RA}$ treatment, but initiate the differentiation again upon the second wave of RA treatment. Therefore, this result suggested more RA treatment (also see the reference) for the low Pax6 expression is necessary.

By examining differentiation markers, upregulation of NE cell lineage was confirmed (Figure 2.2D). Pax6 was expressed at comparable levels in RA and control groups from day 1 to day 3 , but between day 3 and 5, the control group began expressing it at higher levels than the RA group. This trend reversed from day 5 to day 7 . When examining the expression of Nestin, it is clear that the RA 
group produced it significantly more from day 1 to day 5 . After day 5 , Nestin expression grew exponentially, far faster than in the control group. This may indicate that the use of RA on day 0 has its most profound impact on Nestin expression only when the neural stem cells are differentiating into precursor motor neurons. MAP2 and DCX, both neural markers, demonstrated similar trends in the quick growth of their expression levels in the RA group that far outpaced the expression of the control group from day 1 to day 7 . All four of these markers are indicators of an ectoderm differentiation pathway and are thus desired to grow in intensity to demonstrate neuronal lineage. $T$ represents mesoderm marker, which was never significantly expressed, indicating that the pathway was never pursued. Endoderm marker Sox 17 was expressed higher in RA group than control, indicating that RA may also promote endoderm expression.

After examining the cell viability, as measured with Resazurin, every other day for 11 days, it is clear that the RA treatment strongly promoted the survival of the cells over the control group (Figure 2.2E). It is intriguing to recognize that the only significant difference in cell viability between the experimental and control groups happened on days $1,7,9$, and 11 . This data indicates that the cell viability is not significantly different during the neural stem cell stage, but grows to significance during the precursor motor neuron and mature motor neuron stages. It could indicate that the cell is far more susceptible to apoptosis during motor neuron induction, and thus the addition of RA on day 0 and later may have a protective long-term effect for the experimental group. 
A

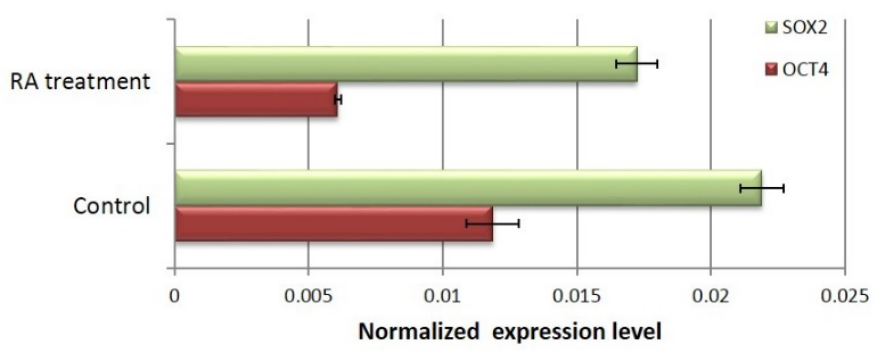

C

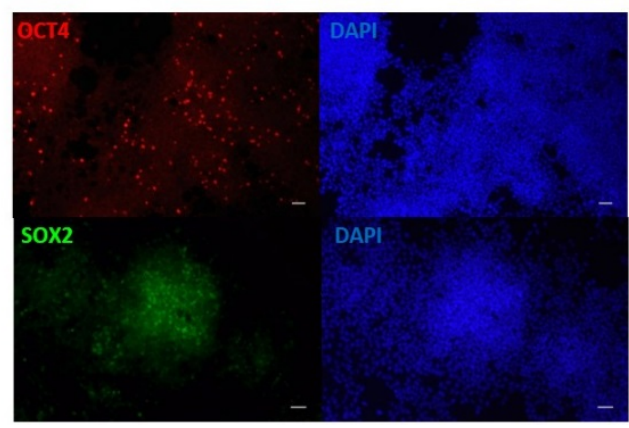

D
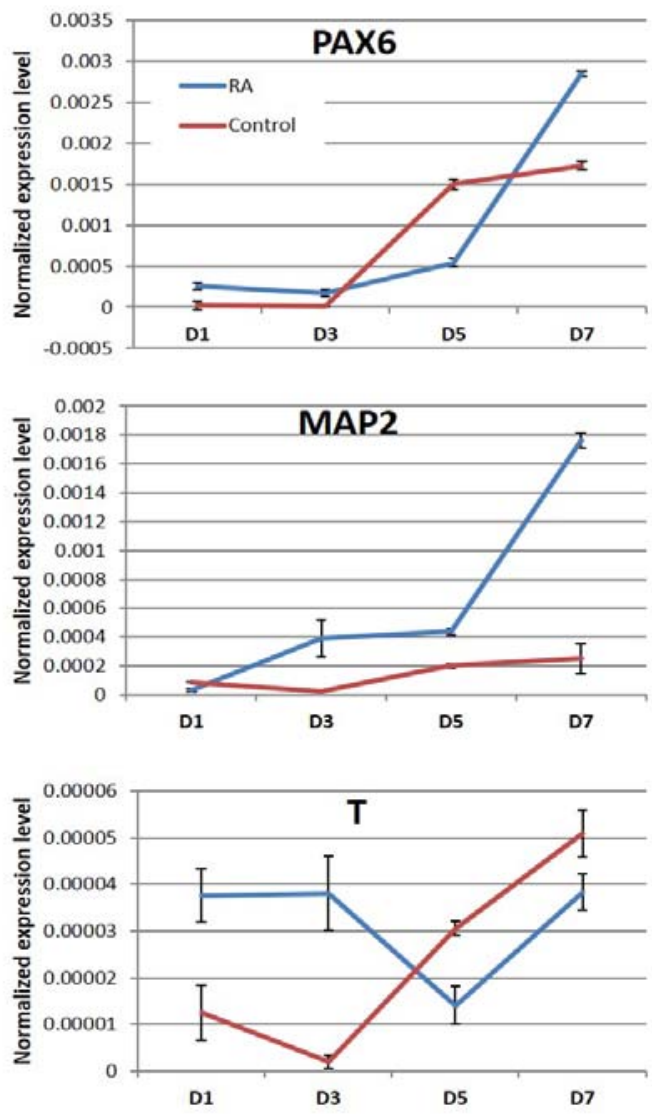

B

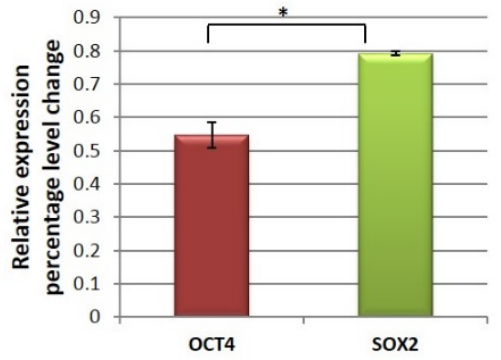

Control
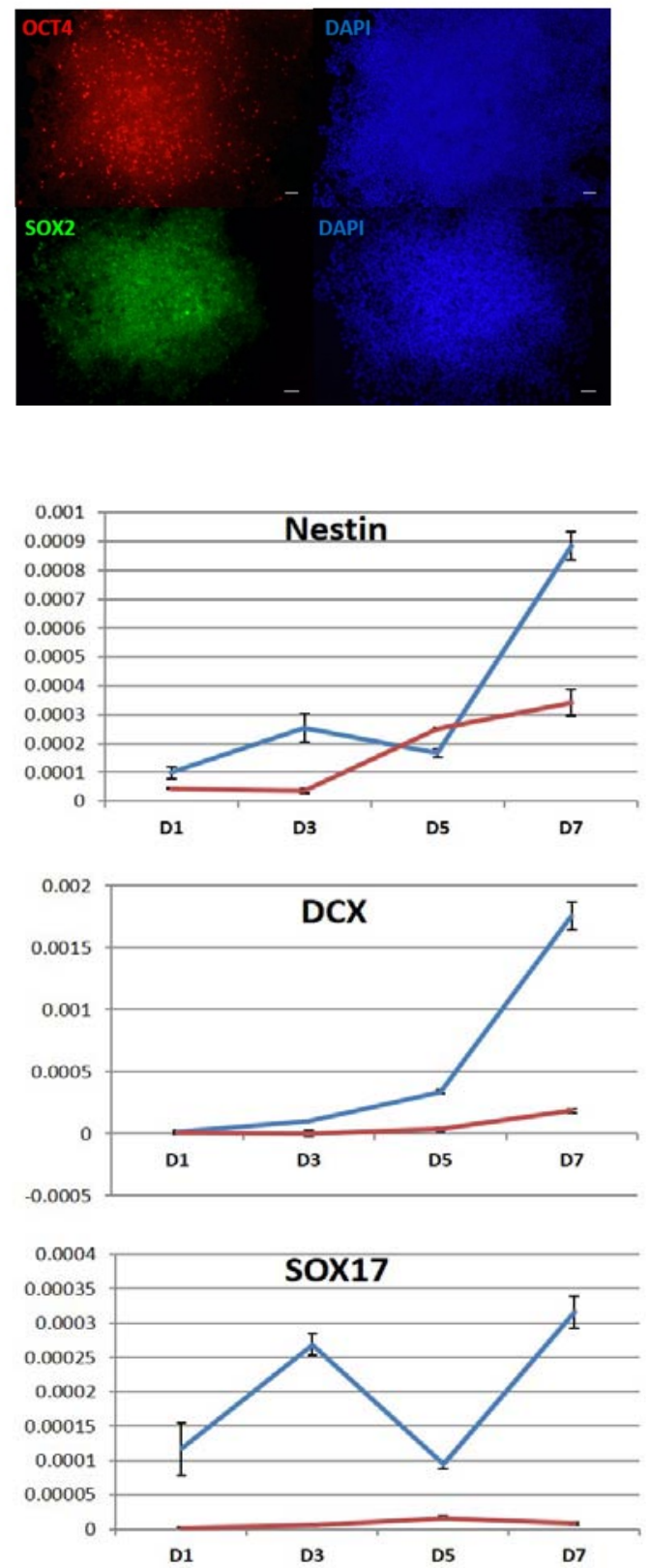
E

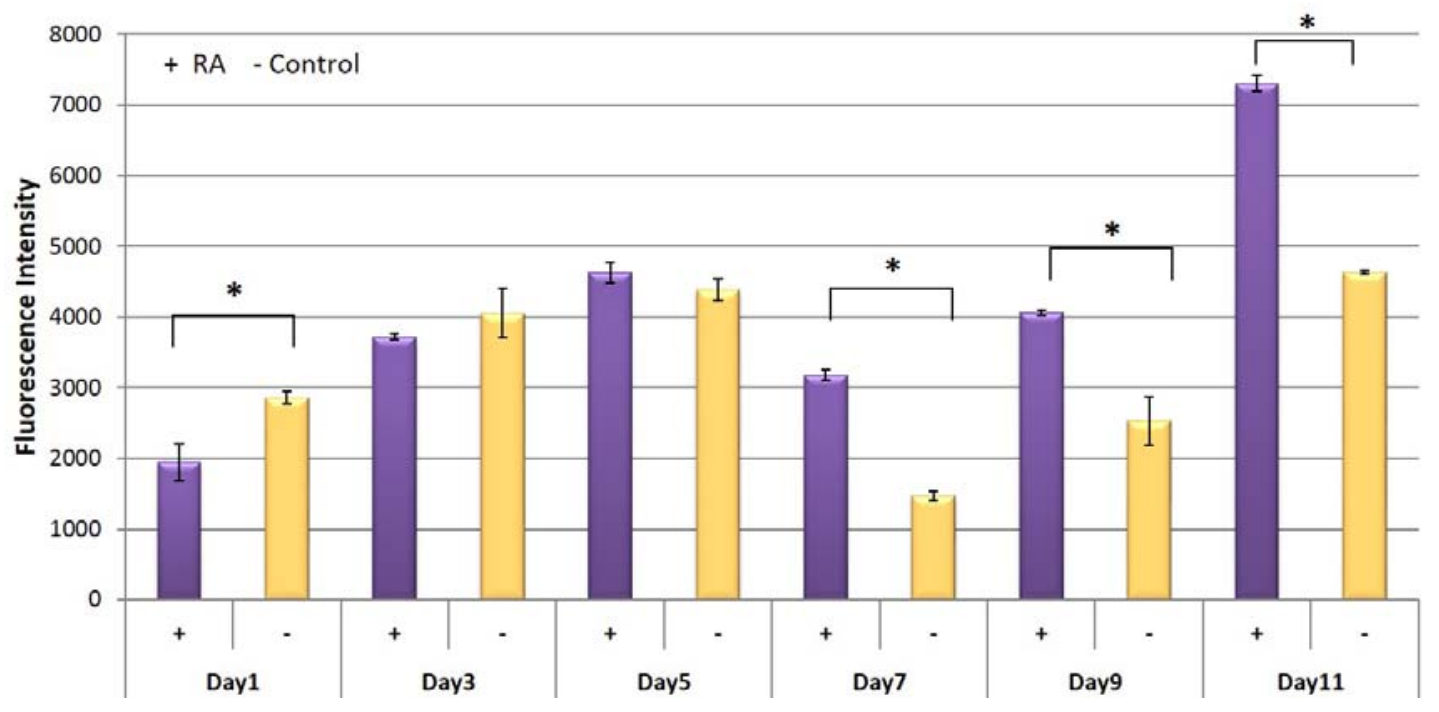

Figure 2.2. Effects of RA introduction on mRNA expression, transcription factors and viability. Expression of pluripotency $(A, B)$ and differentiation (D) Primers in mESCs were assessed by real time-PCR. (A, D) The gene expression level were normalized to the house keeping gene GAPDH expression level. (B) Normalized the gene expression level of RA treatment group on day 1 to day 0 . Average values and standard errors are from two replicate experiments. (C) RA significantly decreased the protein expression of the pluripotency factors. (E) RA increases the Cell Viability. Scale Bar: $100 \mu \mathrm{m}$. Sample number in each group, $\mathrm{n}=3$. Average values and standard errors are from three replicate experiments, ${ }^{*} p<0.05$

\subsubsection{The Effect of NaB on the Conversion of ESCs into Neural Stem Cells.}

The impact of $\mathrm{NaB}$ was examined using real time PCR and Immunocytochemistry. After the fourth day of the protocol, the expected neural stem cell marker Nestin and neural marker MAP2 were expressed $25 \%$ and $37 \%$ higher than the controls, respectively (Figure 2.3A). The mesoderm marker $\mathrm{T}$ was consistently expressed below control levels and the endoderm marker Sox17 was expressed at the same levels as controls by day 4 . Examining the results of Immunocytochemistry on day 4 it is clear that the neural stem cell marker Nestin is far more highly expressed in the NaB trials (Figure 2.3B). 
A

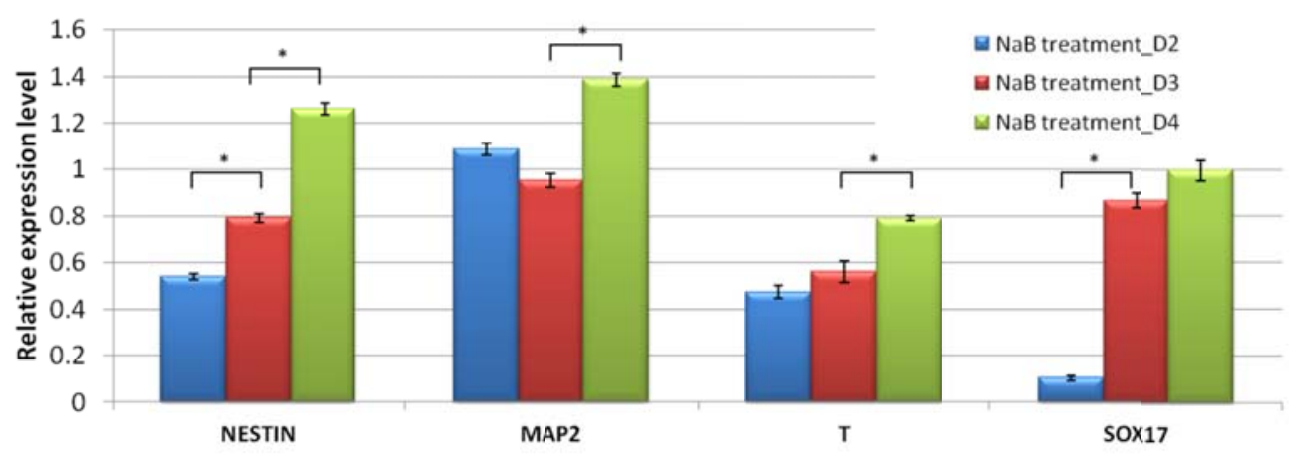

B

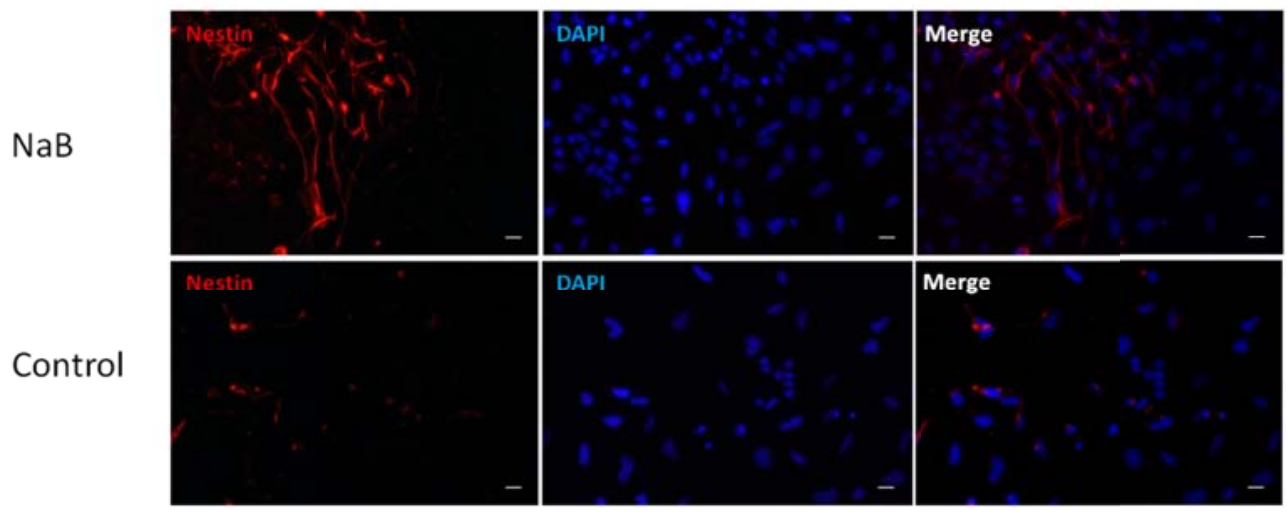

Figure 2.3. NaB treatment induces neural stem cells differentiation of embryonic stem cells. (A) Expression of differentiation primers in mESCs was assessed by real time-PCR and is shown as a fold change relative to the control (equal to 1). Average values and standard errors are from three replicate experiments. ${ }^{*} p<0.05$ (B) Immunocytochemistry of Nestin and DAPI on day 4. Scale Bar: $10 \mu \mathrm{m}$.

\subsubsection{Continued Presence of Genistein and NaF Promotes Motor Neuron}

Generation.

The effects of simultaneous addition of Genistein, $\mathrm{NaB}$ and $\mathrm{NaF}$ (metabolites group) on motor neuron generation as compared to the control group were examined (Figure 2.4A). Motor neuron precursor markers Olig2 was highly upregulated on day 7 in metabolites group compare to the control. It dropped below the control on day 9 and was expressed 2.8 -fold higher than the control in the metabolites group. Motor neuron marker ISL1 was highly 
expressed on day 7 while motor neuron marker HB9 was drastically upregulated on day 11 in metabolites group. ChAT, a mature motor neuron marker, consistently displayed a significantly higher expression compared to the control group, indicative of the fact that the use of this array of metabolites can quickly create mature motor neurons.

Representative staining in metabolites and groups using antibodies against MAP2, ChAT and HB9 are demonstrated in Figure 2.4B through Immunocytochemistry. Examining the results of Immunocytochemistry, it is clear that HB9 and ChAT are far more strongly expressed in the metabolites group than in the control group. Ratios of HB9+ and ChAT+ cells are significantly different between control and metabolites treated group (Figure 2.4C). 
A
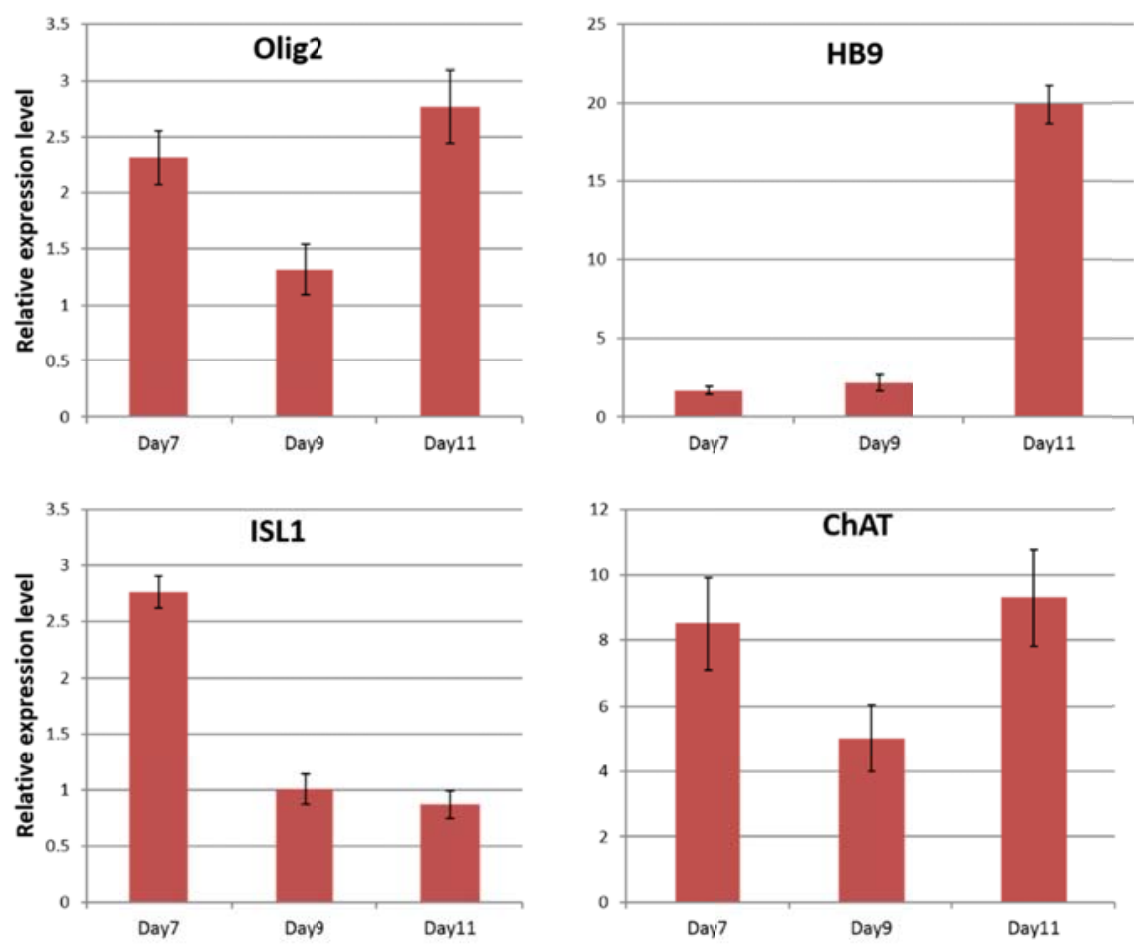

B

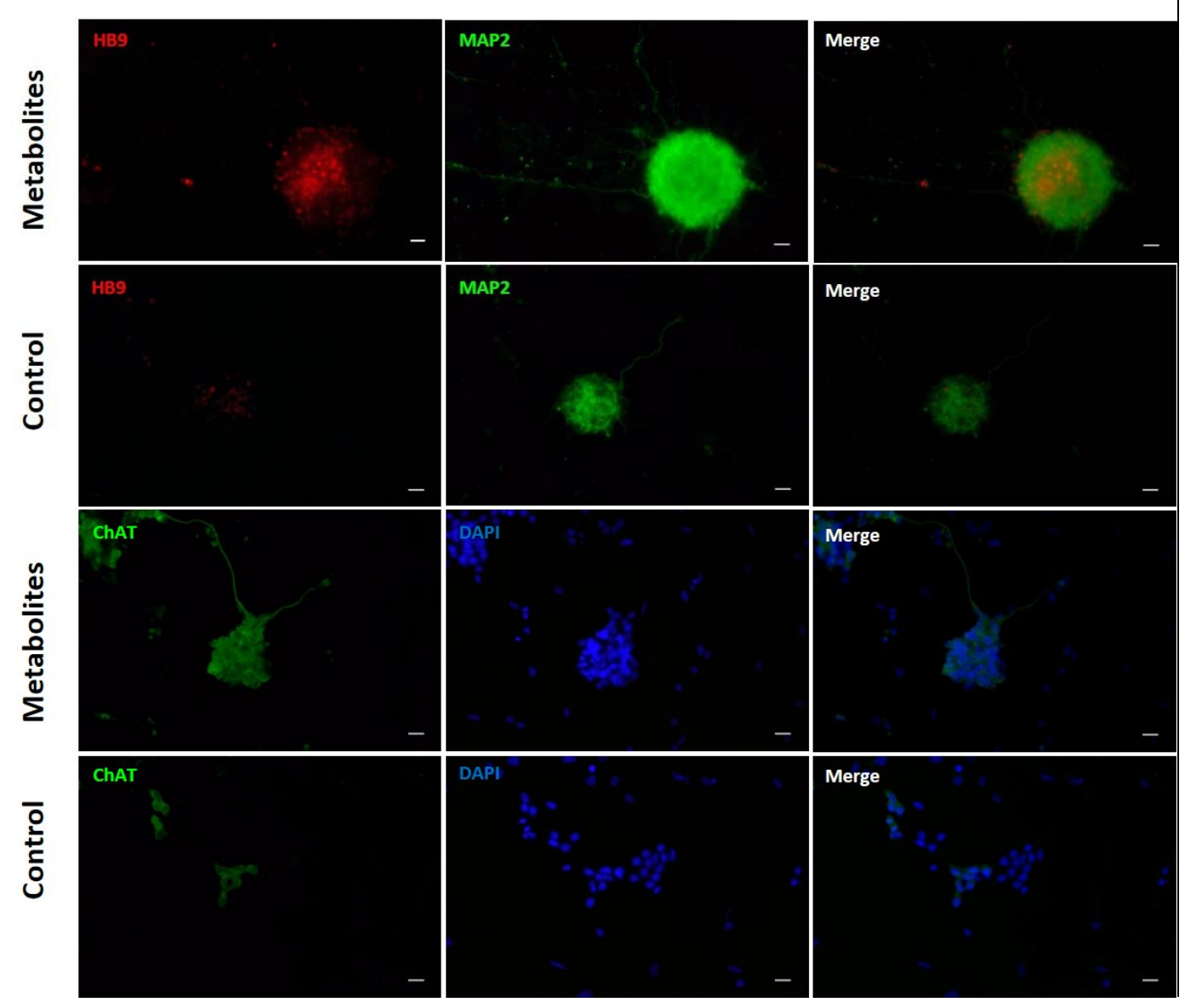




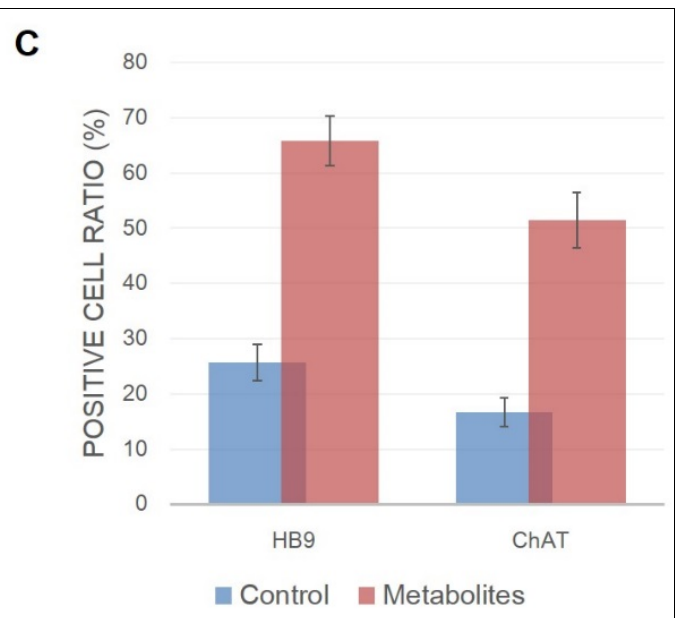

Figure 2.4 Continued presence of Genistein and NaF promotes motor neuron generation.

(A) The expression of MN specific genes such as ISL1, Olig2, HB9, ChAT were upregulated by the treatment of metabolites. The genes were shown as a fold change relative to the control (equal to 1). (B) Immunocytochemistry images of MAP2, HB9 and ChAT positive cells in both groups on day 11. Scale Bar: $10 \mu \mathrm{m}$. (C) Metabolites treatment greatly increased HB9+ and ChAT+ cells. Average values and standard errors are from three replicate experiments, $p<0.05$

\subsubsection{Effects of Various Combinations of Three Metabolites}

While the previous results demonstrate the efficacies of Genistein, NaB and $\mathrm{NaF}$, additional tests were performed to determine the effects of any interactions and the ideal combination, if one existed. Fundamentally, whether particular combinations of these three metabolites would be ideal in maximizing mature motor neuron differentiation efficiency and viability were studied.

To analyze the effects of specific combinations of the metabolites, the expression of different markers including Olig1, GFAP, Olig2, ISL1, HB9 and ChAT were measured (Figure 2.5). Olig1 and GFAP are Oligodendrocyte and Astrocyte markers, respectively, and expression of them was undesired for the purposes of this experiment. Surprisingly, the combination of Genistein and $\mathrm{NaB}$ specifically generated a large quantity of GFAP expression. The most desired expression marker was ChAT, which indicates a mature motor neuron. It is clear 
that a combination of all three markers demonstrated a 12-fold increase in the expression of ChAT over the control group, which had none of these markers. Olig2 was relatively unaffected by this combination compared to controls. In the case of ISL1 and HB9 expression, no combination of these metabolites was significantly superior to any other. It is clear that the most highly desired marker (ChAT), that for mature motor neurons, was significantly impacted and its expression improved by the addition of the three metabolites.
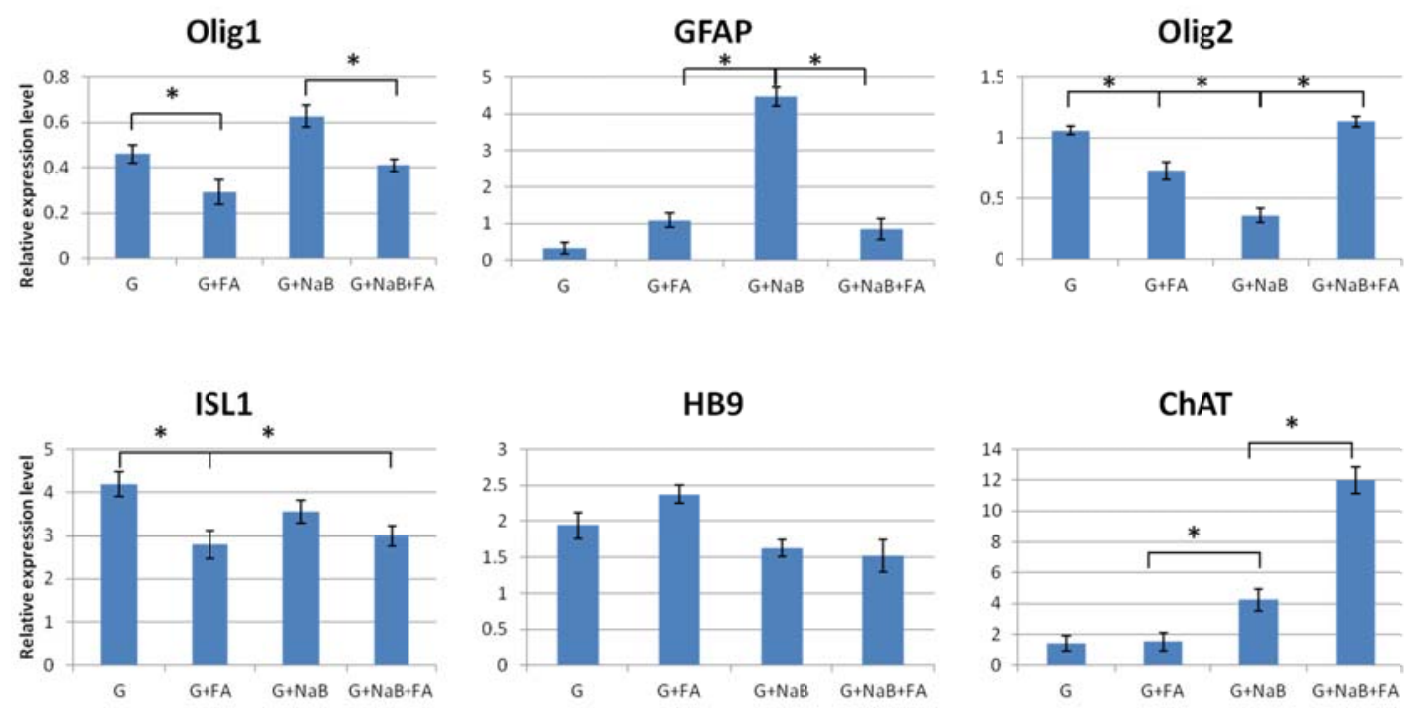

Figure 2.5. Different metabolites effects on day 9. All results are presented as relative expressions as compared to control groups that received none of these metabolites. ${ }^{*} p<0.05$

\subsubsection{Purmorphamine Replaces SHH for Motor Neuron Differentiation.}

PUR has been used interchangeably with $\mathrm{SHH}$ to induce neural differentiation in recent literature. Therefore, the impacts of this small molecule to assess whether its effects are truly analogous to SHH were examined. PUR was utilized in addition to the array of the three metabolites in order to study 
whether a low-cost protocol utilizing primarily metabolites could be as effective as more traditional techniques.

Examining the results of real time PCR in Figure 2.6A, it is clear that the expression of Olig2, ISL1, HB9 and ChAT are reliably upregulated by both SHH and PUR. While the relative expression levels provided by each group may slightly differ, this experiment demonstrates the ability of PUR to analogously induce the expression of transcription factors and enzymes similarly to that of $\mathrm{SHH}$. The Immunocytochemistry results presented below in Figure 2.6B demonstrate the colocalization of the transcription factor HB9 and the microtubule-associated protein MAP2 in the PUR group. In addition, the localization of the ChAT enzyme in the mature motor neurons is shown in Figure 2.6B. These results are shown to be similar to those displayed in Figure 2.4B.

A
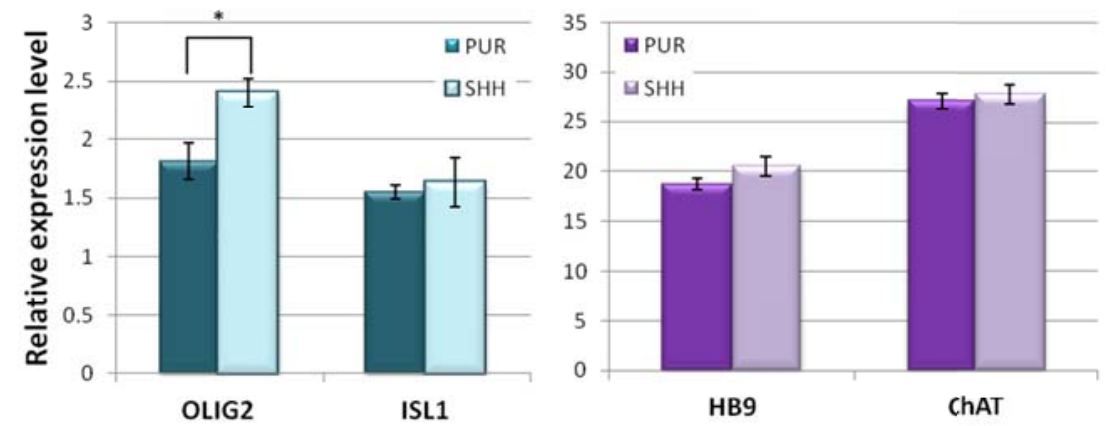

B

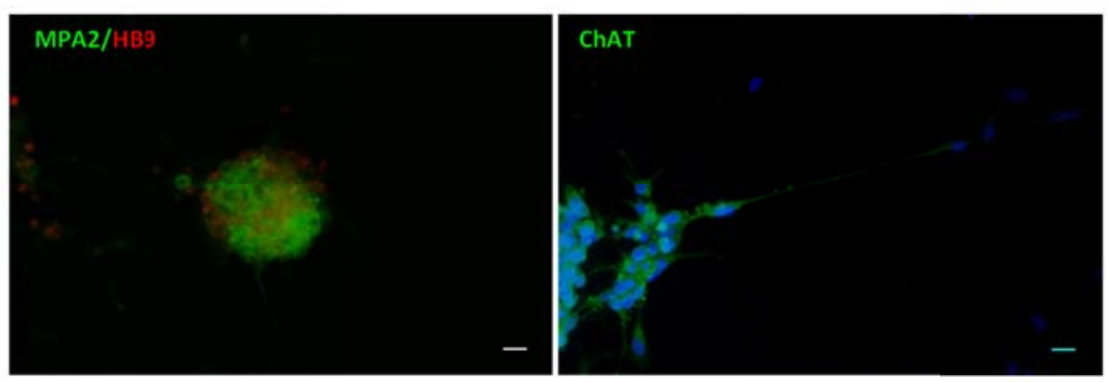


Figure 2.6. Efficient generation of motor neurons by PUR. (A) Quantitative real time PCR analyses (day 12) indicated expression of motor neuron progenitor and motor neuron markers by differentiated neural stem cells that were cultured with RA or PUR from day4. Values provided are relative expression levels compared to control group values, which are normalized to 1 (B) Immunocytochemistry images of PUR trial showed similar protein expression level of neural/motor neuron markers. Average values and standard errors are from three replicate experiments. Scale Bar: $10 \mu \mathrm{m}$

\subsubsection{Functional Features of ESC derived Motor Neuron.}

The presence of an action potential that mimics native neuron is one of the clearest indications of the success of the culturing and cell-type derivation strategy. Each action potential recorded occurred following the stimulus, after which baseline voltage levels were reacquired and maintained. The resting potential of each of these trials immediately prior to the action potential averaged to $-72.3 \mathrm{mV}$, almost a perfect mimic of the resting potential of native neuron (-70 $\mathrm{mV}$ ). In addition, the depolarization/repolarization phase and hyperpolarization phase both lasted approximately 1 millisecond apiece, akin to native nerve cells [145].

While a typical neuronal action potential causes a depolarization to $30-50$ $\mathrm{mV}$, extracellular measurements typically record weaker signals due to the dissipation of the strength of the generated electrical field as it travels through the medium towards the microelectrode. Although the measurements were made extremely close to the target neurons, the action potentials measured prior to amplification were only about $1 \mathrm{mV}$. Fortunately, previous literature supports these values, as a study of retinal ganglion cells in vitro generated recorded potentials in the 1-2 $\mathrm{mV}$ range with a similar pulse stimulation setup [146]. While 
the initial measurements were noisy, a moving-box average was performed on the data set to smooth it with a box width of 5 .

Single monophasic (positive) pulse stimulation was found to be most effective at inducing action potentials in plated motor neurons. Only a single action potential was observed following stimulation - there were no chains of action potentials. To confirm the validity of these results, two control experiments were performed. In the first control experiment, passive extracellular measurements of the neurons were observed to confirm that the observed action potentials were not artifacts intrinsic to the recording system. In the second control experiment, stimulation and subsequent recording identical to the experimental protocol was performed in a dish cultured with mESCs to confirm that the observed potentials were not artifacts of the stimulation schema.

A

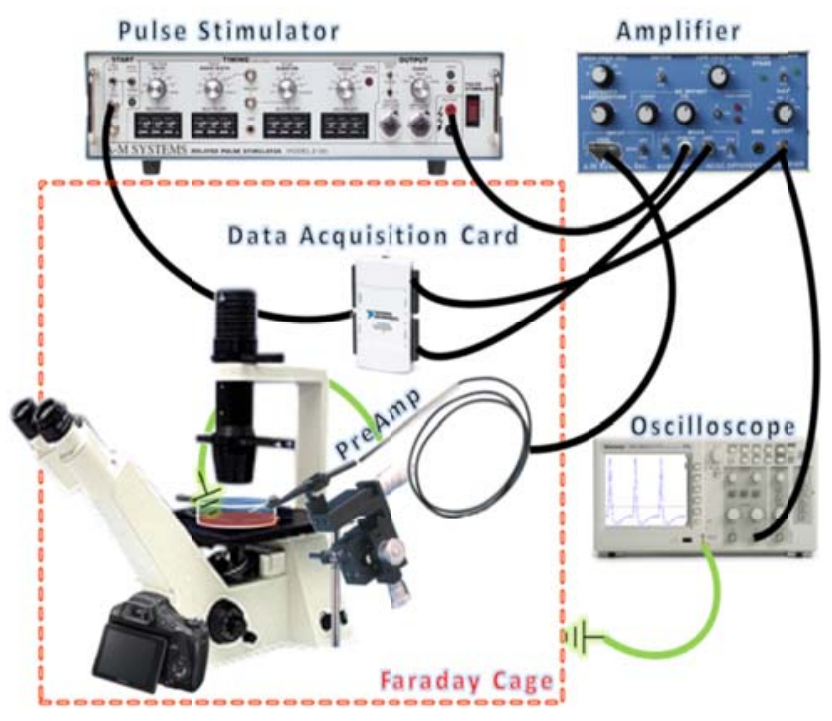


B
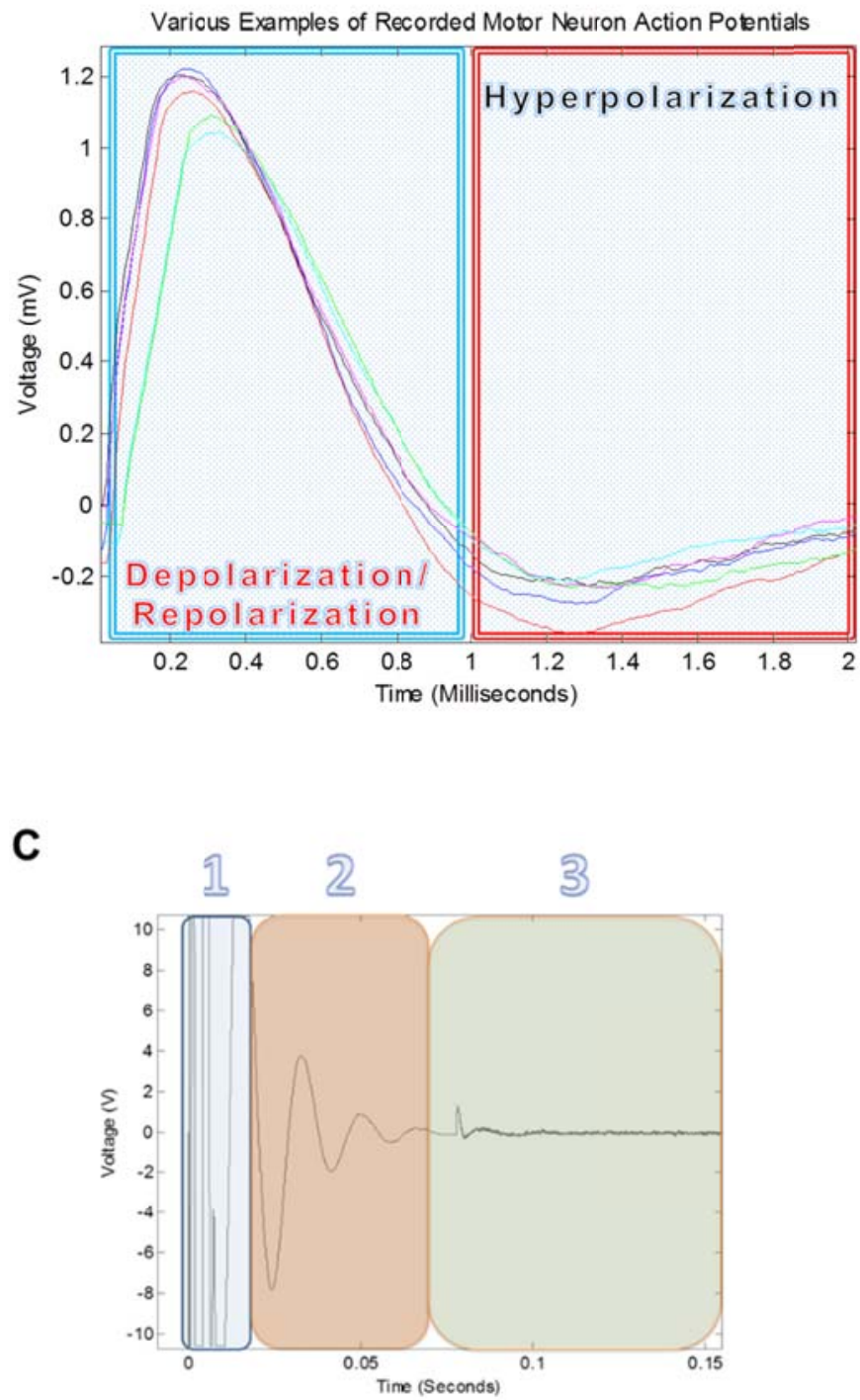
D
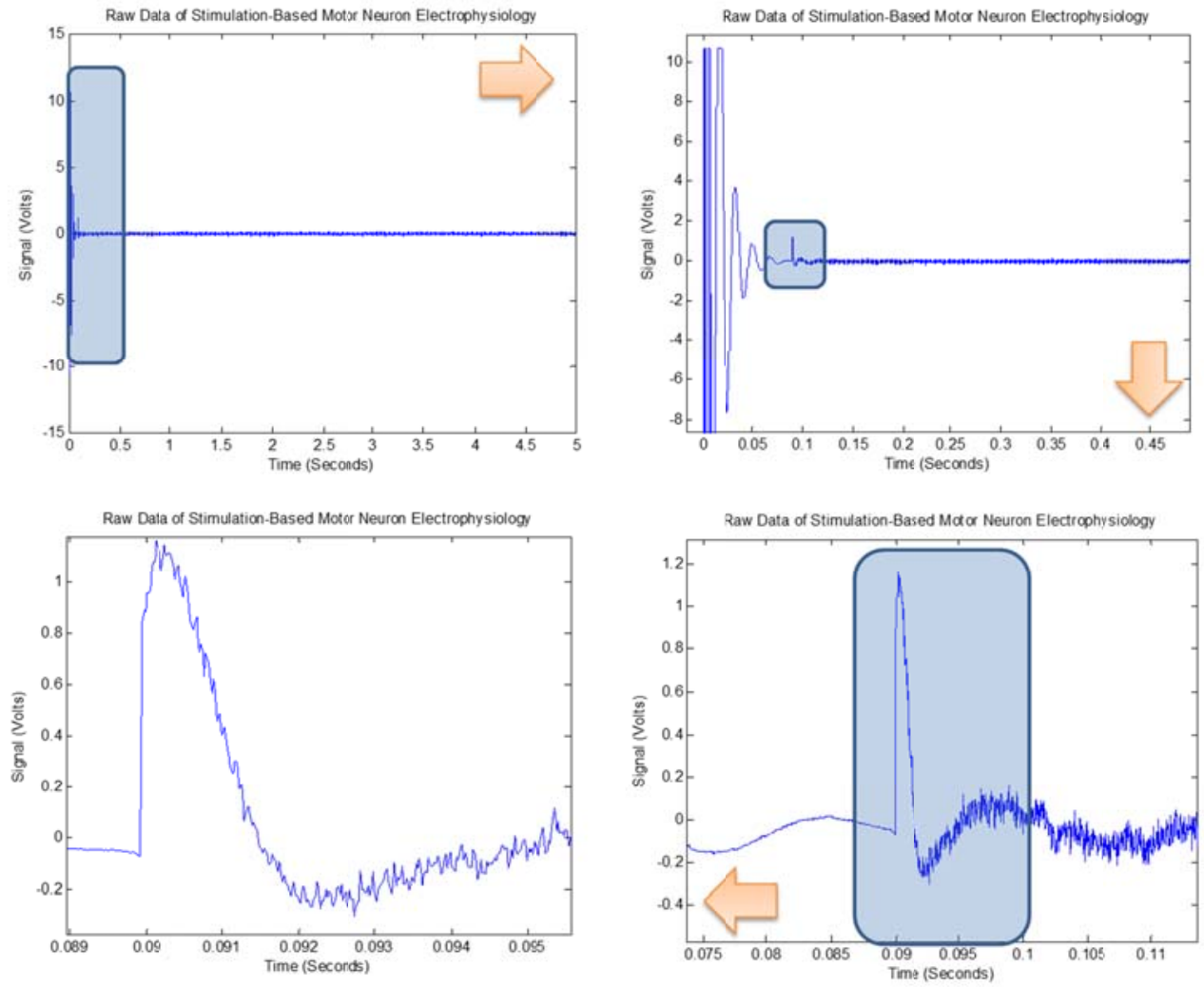

E
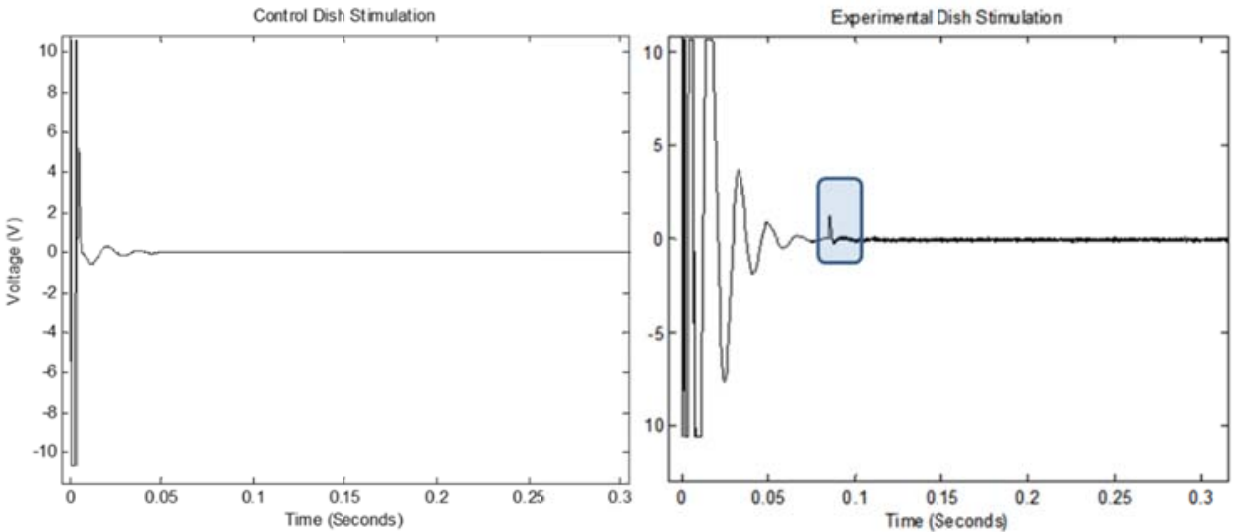

Figure 2.7. Electrophysiological analysis of motor neurons. (A) The electrophysiology setup, documented here in detail, demonstrates the use of a Faraday Cage to reduce aberrant noise. In addition, the grounding setup was specifically optimized to reduce ground loop noise. (B) Due to the nature of the instrumentation setup, stimulation based recordings characteristically had three different components. When the positive monophasic voltage is injected, the DAQ card records a clipped $+/-10 \mathrm{~V}$ signal over approximately $20 \mathrm{~ms}$ (Phase 1). As the DAQ card instructs the amplifier to switch from stimulate to record mode, the recorded signal sinusoidally returns to 
baseline (Phase 2). Once the signal has returned close enough to baseline, a neuronal action potential can be seen (Phase 3). (C) Various recorded motor neuron action potentials are overlaid, each from different neural cell clusters in different dishes, demonstrating the overall homogeneity of mature motor neuron action potential properties. Stimulation occurs immediately prior to the beginning of the displayed recordings. (D) A side by side comparison of a stimulation-based recording of a control dish (mESCs) and experimental motor neuron dish (blue rectangle indicates action potential) using the same parameters demonstrates the ways in which the media and surrounding cells modulate the instrumentation's return to baseline.

\subsection{Discussion}

Incorporating RA into the growth medium 24 hours before switching to differentiation medium is a critical step for mouse motor neuron induction. During ESCs division, one or both daughter cells maintain the stem cell phenotype, and it is controlled by both intrinsic and extrinsic mechanisms. Stem cells divide either symmetrically or asymmetrically. The growth medium provides different external signals that will keep a balance between symmetrical and asymmetrical divisions and maintain an appropriate numbers of stem cells. In other words, the pluripotent circuitry is not an independent entity, but rather a regulatory system which maintains ESCs and chooses which induction pathway to pursue. Incorporating RA into the medium breaks this balance and forces more stem cells towards ectoderm linage. In addition, comparing to mESCs, RA-treated

ESCs are less sensitive to the existence of growth factor. Therefore, when the LIF is withdrawn from the medium, cells have a higher tolerance to the new medium thus preventing them from apoptosis because of the environment change.

The impact of $\mathrm{NaB}$ as a metabolite inductive factor was clearly demonstrated by the upregulation of neural stem cell markers Nestin and neural marker MAP2 as compared to controls that did not receive the NaB treatment 
(Figure 2.3A). While the mesoderm factor was significantly downregulated in comparison to the expected ectoderm factors, the endoderm factor Sox17 was maintained at similar levels in both the treatment and control group. The maintenance of endoderm factors in addition to ectoderm factors prior to motor neuron differentiation likely has an evolutionary origin. The germ layer, the primary layer of cells which form during embryogenesis, produce three layers in humans (ectoderm, endoderm, mesoderm), however in vertebrates without bilateral symmetry only two layers (ectoderm and endoderm) are produced [146]. It is clear that evolutionarily the ectoderm and endoderm are more tightly related, which likely causes the similarity in ectoderm and endoderm markers expression levels during the initial induction period towards immature neurons.

While the use of Noggin is traditionally essential for neural tube development or neural cell induction, the impacts of using Noggin alone versus using it alongside the three metabolites were investigated here. Noggin is a nonmetabolite essential signaling molecule that promotes patterning during embryonic development, and in the absence of BMP4 it will cause the patterning of the neural tube. This is why BMP inhibitors are essential in this study. Examining the results of real time PCR, the most important marker of success is the expression of ChAT, which indicates the presence of a mature motor neuron. While using Noggin alone generated a 3-fold increase in the expression of ChAT by day 7 over controls which did not receive Noggin, the addition of the three metabolites generated an expression of ChAT that was almost 24 times as strong as the control group (Figure 2.4). Another strong indicator of the success 
of this experiment was the expression of HB9, another motor neuron marker. By the 11th day of the protocol, while the Noggin group only had twice the level of expression as compared to controls, the combination of all three metabolites was expressed almost 30 times as strongly. It is evident that the metabolite approach is significantly quicker for obtaining mature motor neurons by examining the interplay between the Olig2 expression, an immature motor neuron marker, and ChAT, a mature motor neuron marker. On day 7 of the experiment, the expression of Olig2 was far higher expressed in the $\mathrm{N}$ group than in the $\mathrm{N}+\mathrm{M}$ group, while at the same time the expression of ChAT was significantly greater in the $\mathrm{N}+\mathrm{M}$ as compared to the $\mathrm{N}$ group. It is evident that the addition of these metabolites to the Noggin improves the speed and efficiency by which these cells are induced into motor neuron lineage.

While the efficacy of the metabolite approach has been demonstrated through the previous experiments, whether there was any interplay within this group of metabolites was considered to determine if there was a possibility that a sub-combination of the three metabolites would be more efficient than using them all. After carrying out real time PCR using various combinations, it became clear that for the most highly desired marker, the mature motor neuron marker ChAT, the combination of all three metabolites was significantly more expressed than any sub-combinations or a control group that received no metabolites by day 9. Examining the expression of the motor neuron progenitor, Olig2, there was little difference in its expression as compared to controls. Since this is already day 9 , this may be an indication that most of the immature motor neurons 
had already matured by this point. While the motor neuron marker ISL1 is not as much of an indicator of maturity as ChAT, its expression was not significantly different between the groups either.

One surprising result from this analysis was the unexpected upregulation of GFAP, an astrocyte marker, when the specific combination of Genistein and $\mathrm{NaB}$ was used. GFAP was expressed 4 times as much with this combination as opposed to the combinations of Genistein and $\mathrm{NaF}$ or Genistein, $\mathrm{NaB}$ and $\mathrm{NaF}$. At first glance, this data seems to indicate that the combination of $\mathrm{NaB}$ and Genistein plays a catalytic role in astrocytic induction, which can be suppressed through the addition of NaF. To examine the potential method of action, a commercially available astrocyte differentiation media, Gibco Astrocyte Medium (Gibco) was first examined. A particular component of it added in catalytic amounts is called sodium selenite. Sodium selenite is used in astrocyte differentiation media to protect the cells against oxidative stress, a process that simultaneously aids in the induction process. Based on the fact that only the combination of Genistein (oxidative stress reducer) and $\mathrm{NaB}$ (neural lineage differentiation assistor) upregulate the GFAP marker, while NaF (another oxidative stress reducer) inhibits the effect, it seems that the origin of these results may lie in the mechanisms of action of $\mathrm{NaF}$ and Genistein. While $\mathrm{NaF}$ works through activation of the Nrf2 antioxidant pathway, Genistein acts through the inhibition of protein phosphorylation at tyrosine residues. While analysis of the differences in mechanism of action between these two processes may hold 
promise in uncovering a metabolite approach to astrocytic induction, further studies will be needed to investigate this.

Real time PCR was used to confirm analytically that there was any difference between the expressions of cells cultured in PUR to those in SHH. Examining four markers previously described, Olig2, ISL1, HB9 and ChAT, it could be seen that there was an insignificant difference between groups in the production of mature motor neurons, as measured using ChAT. The only significant difference between the groups was in the expression of the immature motor neuron marker Olig2, but since the difference is minute and ChAT was comparably expressed it is believed that these molecules are truly interchangeable.

While real time PCR and Immunocytochemistry are valuable molecular techniques for characterizing differentiated cells, electrophysiology was essential for demonstrating that the mature motor neurons could be electrically stimulated and generate typical action potentials. Fortunately, the resting potential, peak voltage and timescale of neuronal action potentials was all shown to mimic native tissue. With the addition of electrophysiological analysis, the mature motor neurons have been demonstrated to mimic native tissue functionally, structurally and molecularly.

By choosing metabolites that affect stem cell metabolism in known ways, a highly effective protocol was derived. We have mentioned previously how $\mathrm{NaF}$ acts as an antioxidant by stimulating the Nrf2 antioxidant pathway, but previous literature has shown that the mechanism of this pathway involves the redirection 
of glucose and glutamine away from glycolytic pathways towards more anabolic pathways [147]. Clearly, the actions of NaF induce the differentiation of motor neurons from ESCs through this metabolic modification. The effects of Genistein can also be shown to have a metabolic origin. Genistein is a phytoestrogen, belonging to the category of isoflavones, which intrinsically have anthelmintic activity that inhibit glycolysis enzymes [148]. Thus it is evident that an origin of Genistein's ability may lie in an underlying metabolic mechanism. To finalize the claims, NaB modulates metabolism must be demonstrated as well. Fortunately, $\mathrm{NaB}$ has been shown to modulate glutamine metabolism by decreasing cell's ability to oxidize glucose, shifting the metabolism away from glucose and towards the oxidation of butyrate [149]. RA was also an essential component of this study, and numerous studies have recognized that it induces a metabolic shift from glycolysis towards oxidative phosphorylation [150]. Through these claims, it is clear that the array of small metabolites not only has been proven to help with cell induction and survival, but also share an underlying metabolic mechanism. 
CHAPTER 3 Development of Electrospun Fiber Scaffolds using Poly (glyceroldodecanedioate)/Gelatin Blended for Neural Tissue Engineering

\subsection{Introduction}

The definition of tissue engineering in simple terms is to fabricate a tissue construct mimicking the structural and functional characteristics of native tissues in an in vivo environment. One critical success factor of such an endeavor is to fabricate a skeleton, also known as 'scaffold', to imitate the function of the native extracellular matrix (ECM) [151]. ECM in native tissue interacts constantly with cells through various mechanical and biochemical means [152]. Therefore, an ideal scaffolds must meet several requirements that include biocompatibility, mechanical stability, appropriate topography, and presenting proper chemical signals.

Currently, there are three common methods for fabricating nanofiber scaffolds: phase separation, self-assembly, and electrospinning [11]. Among these techniques, electrospinning is the most popular due to its versatility, flexibility and cost efficiency $[153,154]$. The electrospun fibrous scaffolds present an array of characteristics found in native ECM including high aspect ratio, high specific surface area, and high porosity with small pore size. To date, fibrous biomaterials have been generated from collagen [25], gelatin [62], poly $(\varepsilon-$ caprolactone) (PCL) [41], poly(D,L-lactic acid- co- glycolic acid) (PLGA) [15, 16], and poly(L-lactic acid) (PLLA) [14] using electrospinning. It is believed that electrospun fibrous materials will play a critical role in several tissue engineering 
areas such as neural, bone, cartilage, skin, tendon, ligament and cardiovascular tissue engineering [11].

Choosing a suitable material to fabricate tissue scaffolds is the first most significant step for tissue engineering. Popular biodegradable polymers such as poly (lactic acid) (PLA), poly (glycolic acid) (PGA) and their copolymers are exceptionally stiff materials. For soft tissue engineering application, biodegradable elastic materials are desired in order to better match the mechanical properties of soft tissues. Motivated by this need, a biodegradable polyester elastomer poly (glycerol-dodecanedioate) (PGD) was developed by thermal condensation of glycerol and dodecanedioic acid (DDA) (Figure 2.1) [19]. Both glycerol and dodecanedioic acid can be derived from biorenewable sources $[155,156]$ and can be metabolized by human cells as energy sources $[157,158]$, thus both monomers exhibit minimal cytotoxicity.

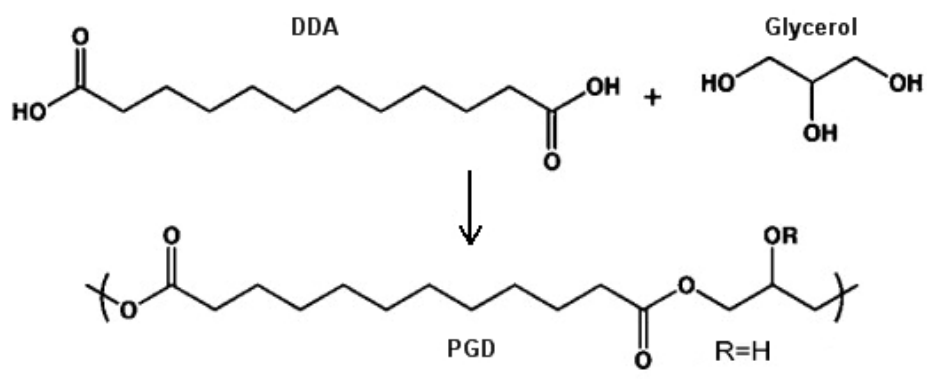

Figure 3.1. Synthetic biodegradable elastomer, poly (glycerol-dodecanedioate).

PGD polymers also possess several beneficial characteristics for soft tissue engineering applications. Stiffer synthetic materials such as poly (hydroxyl butyrate) and poly (L-lactide) have Young's moduli of $250 \mathrm{MPa}$ and $660 \mathrm{MPa}$, respectively, while the biodegradable polymer PGD displays an elastic property 
similar to rubber, with a Young's modulus of $1.08 \mathrm{MPa}$ at temperatures above 37 ${ }^{\circ} \mathrm{C}$, and as such is a close match to the in-situ Young's moduli of the peripheral nerve (0.45 MPa), spinal cord tissue (0.2 MPa-0.8 MPa) and skin (4.2-8.5 MPa) $[18,159-162]$. Moreover, the mechanical and physical properties of PGD can be tuned for different tissue engineering applications by adjusting its chemical composition ratio and fabrication processes. However, like other synthetic polymers, PGD does not carry proper chemical signals to promote cell adhesion and proliferation. To address this shortcoming, adding gelatin, a denatured form of collagen, into a PGD construct is considered.

Gelatin, which is derived from partial hydrolysis of native collagens in animal skin, tendon, cartilage and bone, has been widely used for pharmaceuticals, wound dressings and tissue engineering due to its wealth of merits including biological origin, biodegradability, biocompatibility, formability and low cost [163-165]. However, the strong hydrogen bond interactions in gelatin molecules often leads to a three dimensional macromolecular network. As a result, the mobility of gelatin chains decreases greatly and makes it difficult to be electrospun alone or requires toxic solvent to dissolve [62]. Since PGD can be dissolved in most organic solvents and even in a mixture of ethanol and water, it is interesting to investigate whether PGD and gelatin can be blended and electrospun into fibers to get hybrid scaffolding materials.

Electrospinning is one of the simplest and most versatile techniques to produce micro-to-nanometer size fiber scaffolds from polymers or other materials. The basic principle of electrospinning involves a Taylor cone of 
solution that is held at the orifice of a needle by applying high voltage between the tip of the needle and a grounded collector. When the electrostatic repulsion in the solution overcomes the surface tension, a charged fluid jet is ejected out of the needle tip, travels through the air with solvent evaporation, and is finally deposited on the grounded collector [166]. In this study, an aluminum foil strip was attached perpendicular to a flat metal plate, which ensured a fiber mat with a relatively large area could be collected. The fibers are stretched across the area between the tip of the strip and the metal plate, which forms a fiber array and can be easily transferred to another substrate (Figure 3.2).

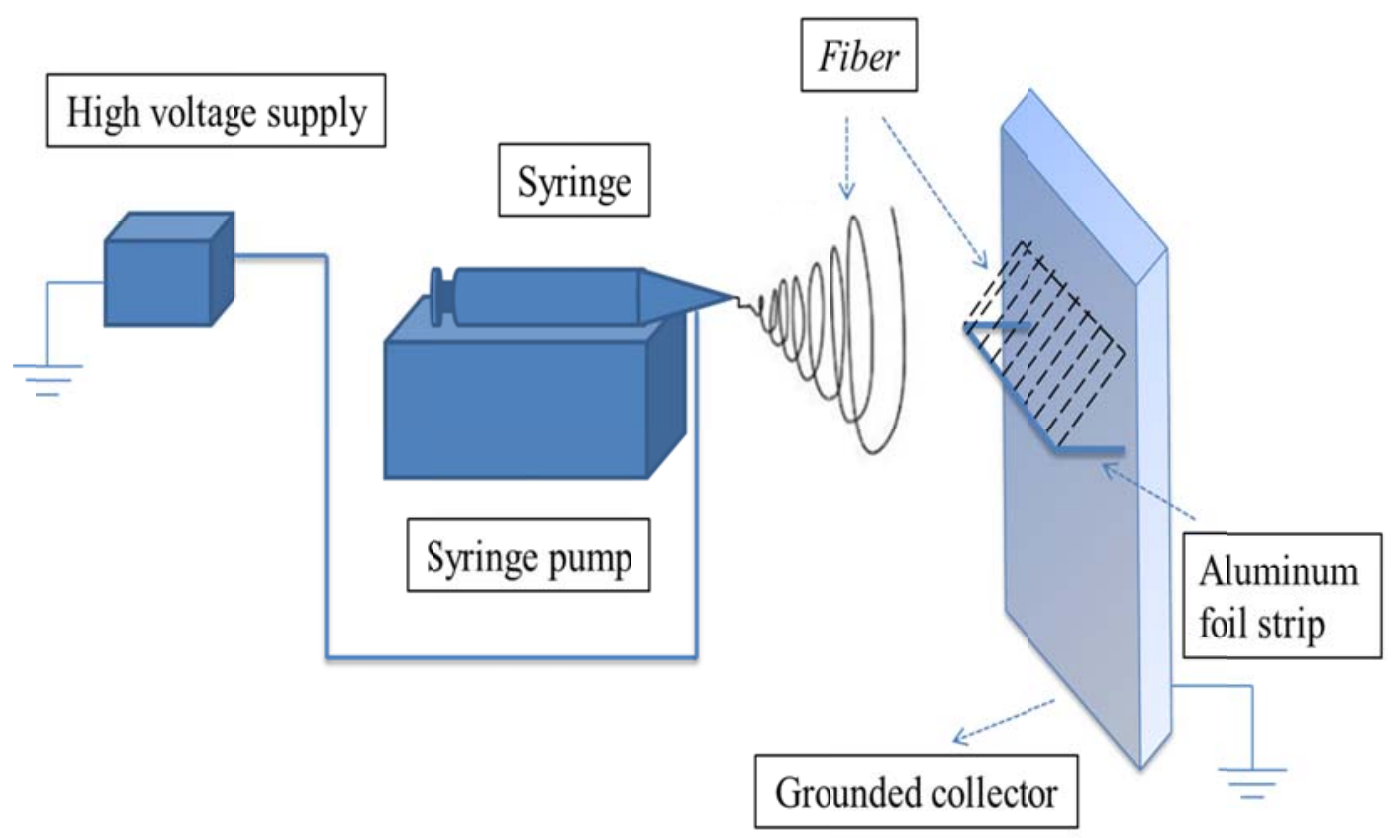


Figure 3.2. Electrospinning setup including a syringe pump, a high voltage power supply and a new designed collector.

\subsection{Experimental}

\subsubsection{Determination of PGD molecular weight by Gel Permeation}

Chromatography (GPC)/Size Exclusion Chromatography (SEC)

A High Performance Liquid Chromatography (HPLC) system (Perkin Elmer, Flexar) equipped with a $25 \mathrm{~cm}$ long GPC column (Jordi Gel DVB Column$5 \mu, 250 \times 4.6 \mathrm{~mm}$, Mixed Bed, Jordi Labs) was used to determine the PGD molecular weight (MW). The analog outputs from both the UV detector and the Evaporative Light Scattering Detector (ELSD) were fed to a data acquisition unit (BIOPAC, model MP100) to be converted into digital signals. Then the acquired signals were processed, displayed and stored in a computer for later analysis.

A polystyrene molecular weight standard kit with the MW ranging from 497 Da to $1.03 \times 10^{6} \mathrm{Da}$ (TSK standard POLYSTYRENE, TOSOH, Japan) was used to establish the calibration curve of MW vs. retention time. For sample preparation, $3 \mathrm{mg}$ of PGD from different time points (24 hours, 72 hours and 96 hours) were dissolved in $3 \mathrm{~mL}$ of Tetrahydrofuran (THF) in a glass vial. The vial was shaken until the polymer has completely dissolved. Then $100 \mu$ sample was loaded into the HPLC system with a syringe. Both analog signals of UV and ELSD were digitalized at a rate of 200 samples per sec. This procedure was repeated three times for each sample. For data analysis, the data acquired from the BIOPAC software were imported into a Microsoft Excel spreadsheet. At each sampled time point, the voltage output of the ELSD was used to calculate the corresponding MW from the established calibration curve by assuming the 
voltage output is proportional to the numbers of polymer (created by 12 polystyrene standards of differing molecular weights) to calculate the log of the molecular weight for that sample point. Taking the anti-log of this gave a molecular weight measurement that was then multiplied by the voltage reading at that sample point. The product of the molecular weight and voltage measurement at each of the bounded sampled points, as well as the voltage measurements, were then summed. Finally, the summed product of molecular weight and voltage was divided by the summed voltages to calculate the number-average molecular weight $\left(M_{n}\right)$ using Equation 3.1:

$$
\mathrm{M}_{\mathrm{n}}=\frac{\sum_{\mathrm{i}=1}^{\infty} \mathrm{M}_{\mathrm{i}} \mathrm{n}_{\mathrm{i}}}{\sum_{\mathrm{i}=1}^{\infty} \mathrm{n}_{\mathrm{i}}}=\frac{\sum_{\mathrm{i}=1}^{\infty} \mathrm{M}_{\mathrm{i}} \mathrm{V}_{\mathrm{i}}}{\sum_{\mathrm{i}=1}^{\infty} \mathrm{V}_{\mathrm{i}}}
$$

where $M_{i}$ is the $i^{\text {th }}$ sampled point's corresponding molecular weight and $V_{i}$ is the $\mathrm{i}^{\text {th }}$ sampled point's voltage measurement.

By taking the same approach, the following equation was used to calculate the weight-average molecular weight $\left(M_{w}\right)$ :

$$
\mathrm{M}_{\mathrm{W}}=\frac{\sum_{\mathrm{i}=1}^{\infty} \mathrm{M}_{\mathrm{i}}^{2} \mathrm{n}_{\mathrm{i}}}{\sum_{\mathrm{i}=1}^{\infty} \mathrm{M}_{\mathrm{i}} \mathrm{n}_{\mathrm{i}}}=\frac{\sum_{\mathrm{i} 1}^{\infty} \mathrm{M}_{\mathrm{i}}^{2} \mathrm{~V}_{\mathrm{i}}}{\sum_{\mathrm{i}=1}^{\infty} \mathrm{M}_{\mathrm{i}} \mathrm{V}_{\mathrm{i}}}
$$

3.2.2 Preparation of $\mathrm{PGD/gelatin}$ polymer solution and fabrication of electrospinning fibers

To prepare the polymeric solution for electrospinning, glycerol and Dodecanedioic acid (DDA) from Sigma-Aldrich (St Louis, MO) were mixed in a molar ratio of $1: 1$ and reacted at $120^{\circ} \mathrm{C}$ for 100 hours to procure PGD polymers. Meanwhile, a mixture of poly(ethylene oxide) (PEO), gelatin, and solvent $(65 \%$ 
ethanol and $35 \%$ water) with a combination of $1.5: 3: 95.5(\mathrm{w} / \mathrm{w})$, which was denominated as the basal solution (BS), was heated in a low temperature oven and stirred every 10 minutes until it became a homogeneous solution. PEO with a high average molecular weight $(600,000 \mathrm{Da})$, when combined with PGD, allowed for synthesis of longer fibers. The testing solutions for making electrospun fibers were composed of $50 \%$ PGD and $50 \%$ BS, $40 \%$ PGD and $60 \%$ BS, $30 \%$ PGD and $70 \%$ BS, and $20 \%$ PGD and $80 \%$ BS, respectively. In addition, $0.1 \%$ riboflavin was incorporated into the polymer solution as a photoinitiator for later crosslinking process. Then the polymeric solution underwent the electrospinning process described in section 2.3. Additionally, in order to evaluate the impact of gelatin on fiber forming and cell attachment, polymeric solutions without gelatin but containing $20-50 \%$ PGD concentration were prepared by dissolving in solvent (65\% ethanol and $35 \%$ water) and adding $2.6 \%$ (w/w) PEO.

In the typical experimental procedure, the polymeric solution was fed into a $5 \mathrm{ml}$ standard syringe and delivered through an $18 \mathrm{G}$ blunted stainless steel needle by a syringe pump (Fish scientific, USA) at a flow rate of $0.6 \mathrm{ml} / \mathrm{h}$. Electrospun fibers were fabricated in an operating voltage of $14.6 \mathrm{kV}$ (SL30, Spellman High Voltage Electronics Corporation, USA), and the distance between the needle tip and the ground collector was $25 \mathrm{~cm}$. An aluminum foil strip was attached perpendicular to a flat metal plate as the electrospinning collector, and fibers formed across the gap between this strip and the plate. In order to get a more uniform fiber mat, the syringe pump was placed at an angle of about 15 
degrees with the horizontal to prevent aggregation of fibers at the front of the strip. After electrospinning, the fibers were exposed to UV light for around 80 minutes to enhance the crosslinking process.

\subsubsection{Characterization of PGD/gelatin fiber scaffolds}

\subsubsection{Scanning electron microscopy (SEM) of Electrospun fibers}

The electrospun fibers of PGD with and without gelatin were analyzed using scanning electron microscopy (SEM, JEOL JSM6330F, Tokyo, Japan) at various magnifications. Before imaging, the fiber samples were placed in a gold sputter coater for 2 minutes to deposit a thin conductive film. The average diameters of the fibers were determined by means of Image $\mathrm{J} 1.47 \mathrm{v}$ software at 100 different locations of the SEM images.

\subsubsection{Raman spectroscopy}

Raman spectra were taken with Raman Micro 400 Series Raman Spectroscopy (Perkin Elmer) using a 50X objective lens and with a 2-second exposure time. These spectrum data were used to evaluate the polymerization process and the chemical structures of PGD, gelatin and their mixture pre- and post-electrospinning. The samples were taped to the shiny side of an aluminum foil covering the microscope slides [167].

\subsubsection{Dissolvability test}

Fiber samples, which had already been exposed to UV light for 80 minutes, were cut into small round pieces of the same size and placed into $60-\mathrm{mm}$ culture dishes. Two milliliters of the Phosphate Buffered Saline (PBS) were added to 
each plate and the plates were kept at $37{ }^{\circ} \mathrm{C}$ for one month to test the dissolvability of the cross-linked scaffolds.

3.2.4 In vitro studies of PGD/gelatin fibers with human foreskin fibroblasts

\subsubsection{Cell seeding}

Human Foreskin Fibroblasts (HFF) cells acquired from Globstem (Rockville, Maryland) were used to test cell viability on the PGD fiber scaffolds with and without gelatin. These cells were cultured in a medium that consisted of 90\% DMEM/F12 basal medium (Thermo Scientific), 1x antibiotics/antimycotics and $10 \%$ fetal bovine serum (FBS) (Sigma-Aldrich, St Louis, MO). Fiber samples made from different compositions were cut into small round pieces of the same size and placed into a 6-well plate with low cell binding surface (Nunc, Fisher Scientific). For pre-seeding treatment, specimens were soaked in PBS for 3 hours and subsequently immersed in fresh medium and kept at $37^{\circ} \mathrm{C}$ overnight. Before seeding, cells were counted using a hemocytometer and then seeded to the scaffolds at a $5.0 \times 10^{4}$ cells/well density. Following the addition of the cell suspension droplet, the fiber mats were kept in an incubator at $37{ }^{\circ} \mathrm{C}$ and $5 \%$ $\mathrm{CO}_{2}$ to allow cell attachment.

\subsubsection{Visualization of cells on the fibers}

In order to visualize cells on the fiber scaffolds, Calcein AM, a cellpermeant dye purchased from Invitrogen (Grand Island, NY) was used to stain the live HFF cells. After culturing the cells at $5.0 \times 10^{4}$ cells/well density for 1 day, $1 \mu \mathrm{l}$ of the Calcein AM reagent was added into $2 \mathrm{ml}$ of cell culture medium and incubated for one hour. Thereafter, the fluorescence signal from the cells was 
visualized under the confocal microscope (D-Eclipse C1, Nikon) by using an excitation wavelength of $488 \mathrm{~nm}$.

\subsubsection{Evaluation of cellular viability}

Resazurin fluorescence dye (Sigma-Aldrich, St Louis, MO), which had been shown to be identical to the commercial assay Alamar Blue [141], was used as an index of cellular viability [168]. After 24 hours cell culturing on the scaffold, the culture medium was drained and $100 \mu \mathrm{l}$ of the reagent were added to each well, mixed with $2 \mathrm{ml}$ of fresh medium. Background controls of $2 \mathrm{ml}$ of medium plus $100 \mu \mathrm{l}$ of the resazurin reagent were also performed. The plate was placed in an incubator at $37^{\circ} \mathrm{C}$ and $5 \% \mathrm{CO}_{2}$ for 4 hours to allow the cells to metabolize the resazurin. Then $100 \mu \mathrm{l}$ medium samples of the different composition of fiber mats as well as the control group (sample numbers in each group, $n=4$; experiment was repeated 3 times), were collected into a 96-well plate in triplicate. Thereafter, the 96-well plate reader was set to measure the fluorescence intensity of a $590 \mathrm{~nm}$ emission with an excitation wavelength of $530 \mathrm{~nm}$. The same procedure was repeated at different time points ( 1 day, 3 days, 5 days and 8 days). Subsequently, the data of fluorescent intensity were converted to cell numbers according to the established standard curve (known cells number v.s. fluorescent intensity), and used to compare the number of metabolically active cells in different samples.

\subsubsection{Statistics}

Analysis of variance (ANOVA) and Turkey's HSD tests were used to evaluate the significance between the PGD with gelatin fibers, PGD without 
gelatin fibers and control group $(n=4)$ for cell viability testing. Kruskal-Wallis Test and Mann-Whitney U-test were performed to determine the statistical difference between groups in fiber diameter distribution. In both cases, the statistical significance was set at $p<0.05$.

\subsection{Results}

\subsubsection{Characterization of PGD polymer}

\subsubsection{Determination of PGD molecular weight}

An HPLC system combined with a GPC/SEC column is the most convenient method to measure the MW of polymers and their MW distributions. In this study, two detectors (UV and ELSD) were used to monitor the elution of polymers. It was found that PGD polymers were almost invisible in the UV detector. Therefore, detection of the elution time for PGD polymers was performed by ELSD. The determined number/weight average molecular weights of PGD at 24 hours, 72 hours and 96 hours are shown in figure 2.3, which were in the order correlated to its overall reaction time. Table 3.1. shows the different $M_{w}$ and $M_{n}$ of PGD and the polydispersity index, $M_{w} / M_{n}$. The larger the index, the wider the polymer molecular weight distribution is [169].

Table 3.1. Number- and Weight- average molecular weight and the polydispersity index of PGD on different time points.

Number-Average Weight-Average

Molecular Weight Molecular Weight Polydispersity

$\begin{array}{lll}\left(M_{n}\right) & \left(M_{w}\right) & \text { index }\end{array}$

$\begin{array}{llll}\text { PGD } 24 \text { hours } & 1354 & 2184 & 1.61249\end{array}$




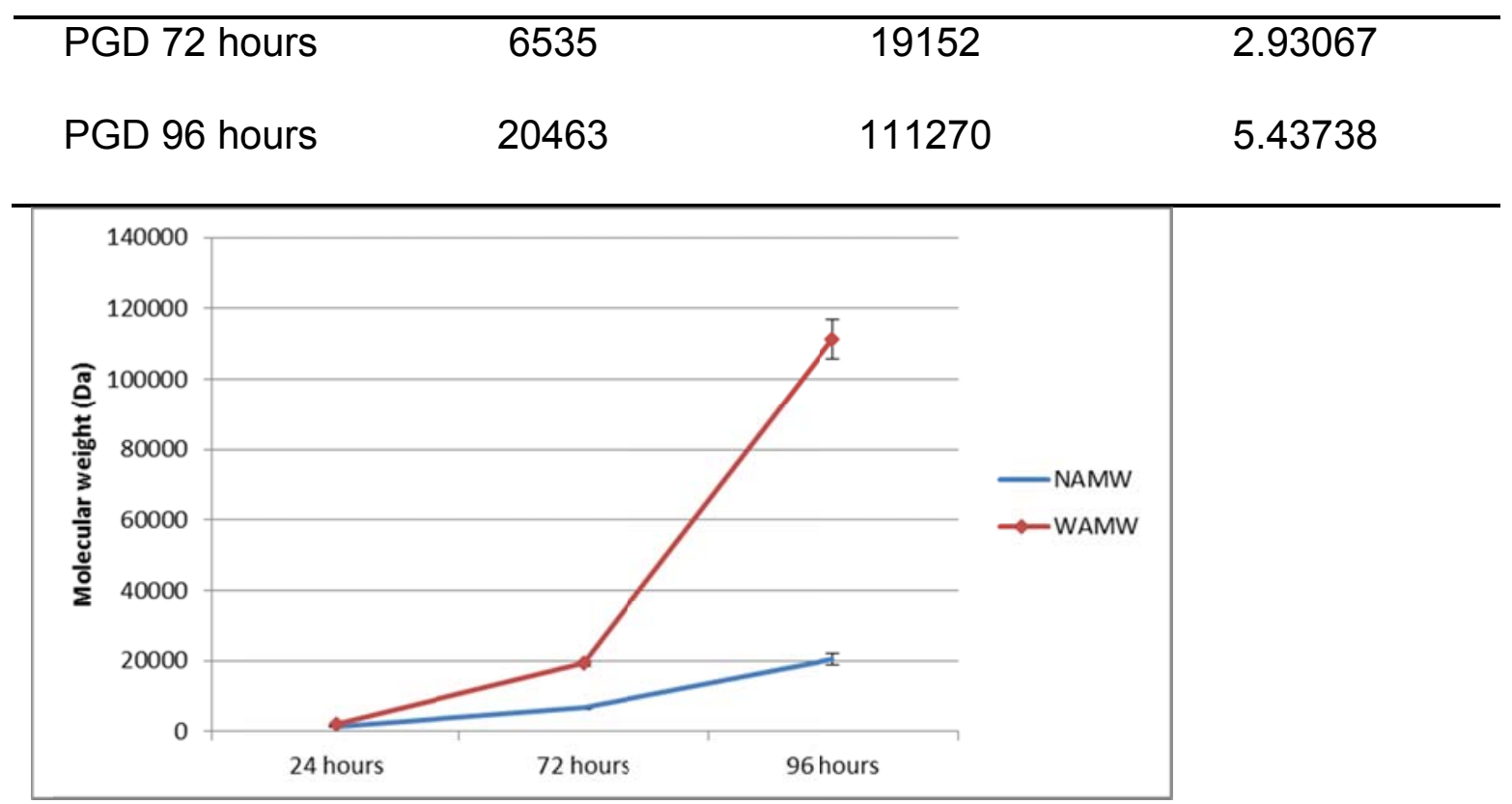

Figure 3.3. Number and weight average molecular weight determinations of PGD. Average values and standard errors are from three replicate experiments.

\subsubsection{Raman spectroscopy}

Raman spectroscopy was used to investigate the polymerization process of PGD and examine whether the electrospinning process affected the primary structure of the PGD polymer. Figure 3.4 shows the spectra of glycerol, DDA and PGD, respectively. During thermal condensation, the carboxylic group in DDA $\left(\mathrm{C}=\mathrm{O}, 1644.60 \mathrm{~cm}^{-1}\right)$ and hydroxyl groups in glycerol $\left(-\mathrm{OH}, 1251.13 \mathrm{~cm}^{-1}\right)$ react to form a new ester bond $\left(\mathrm{C}=\mathrm{O}, 1737.21 \mathrm{~cm}^{-1}\right)$ after removing one water molecule $[170,171]$. The band at $1298 \mathrm{~cm}^{-1}$ and a group of bands in the region between $1400 \mathrm{~cm}^{-1}$ and $1500 \mathrm{~cm}^{-1}$ are due to $\mathrm{C}-\mathrm{C}$ stretching vibrations, and $\mathrm{CH}_{2}$ twist vibrations and deformations, respectively. 


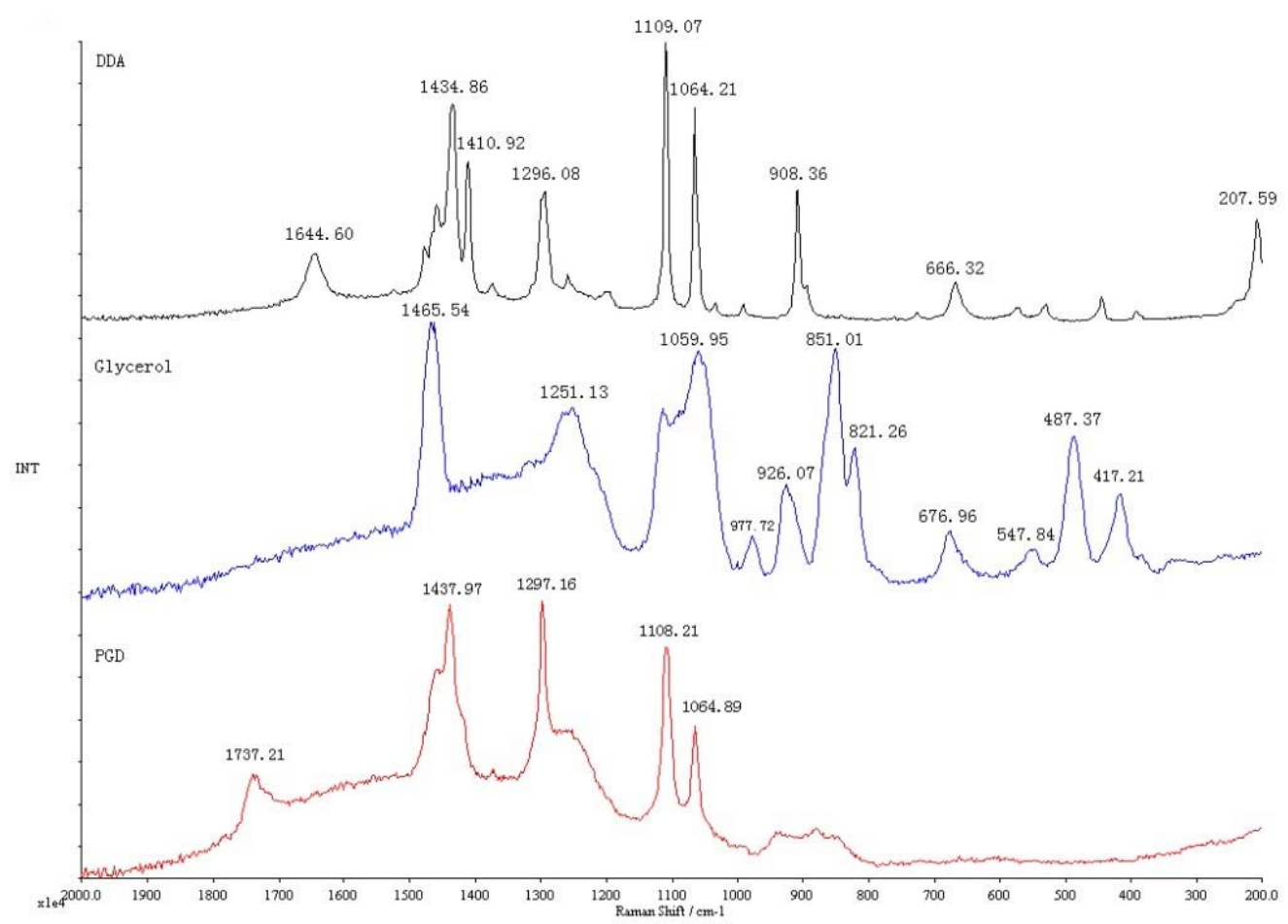

Figure 3.4. Raman spectra of DDA, glycerol and PGD, respectively

\subsubsection{Characterization of PGD/gelatin electrospun fibers}

3.3.2.1 The newly designed collector and the effects of PGD concentration on fiber size

By adopting our newly developed electrospinning collector, PGD was dissolved in $65 \%$ ethanol/ $35 \%$ water and electrospun from the solution containing 20\%-50 \%(w/w) PGD, and the fibers were deposited between the aluminum foil strip and the aluminum plate (Figure 3.5). From figure $3.5 \mathrm{~A}$, it is clear that the fibers were spanning between the strip and the plate, thus the fiber mats could be easily harvested without the risk of damaging to the fibers. In contrast, when using a traditional collector, i.e. a flat metal plate, the fibers 
attached strongly to the collector surface, and it was unable to easily harvest an intact fiber mat. In addition, the aluminum foil strip could be adjusted to fabricate different sizes of fiber mats. For example, the fiber mat could be easily obtained in the size of $9 \mathrm{~cm}$ by $15 \mathrm{~cm}$ as shown in figure 3.5B and 3.5C.

Incorporating $2-3 \%(\mathrm{w} / \mathrm{w})$ gelatin into the polymeric solution with $20-50 \%$ (w/w) PGD was also investigated and found that PGD blended with gelatin can be also electrospun into fibers. Another important observation was that a small amount (1-2\%) of the high molecular weight PEO would result in better long fiber obtainment, therefore PEO was used in all studies. Furthermore, fibrous scaffolds were examined by using SEM to determine the diameters and morphologies of the fibers. One hundred randomly selected areas were chosen from the SEM images shown in figure 3.6 to calculate the average diameters of the fibers in each group. As shown in figure 3.6, the fibers produced from $30 \%$ (w/w) PGD solution had the smallest diameters, with a mean diameter of $0.95 \pm 0.91 \mu \mathrm{m}$. It is observed that when using higher PGD concentration, increased fiber diameters are acquired with mean diameters of $3.33 \pm 1.71$ and $4.93 \pm 7.28 \mu \mathrm{m}$. The results also showed that the polymeric solution with $20 \%$ PGD polymer was not able to produce electrospun fibers. This result indicated that PGD concentration has a significant effect on the electrospun fiber diameter. 


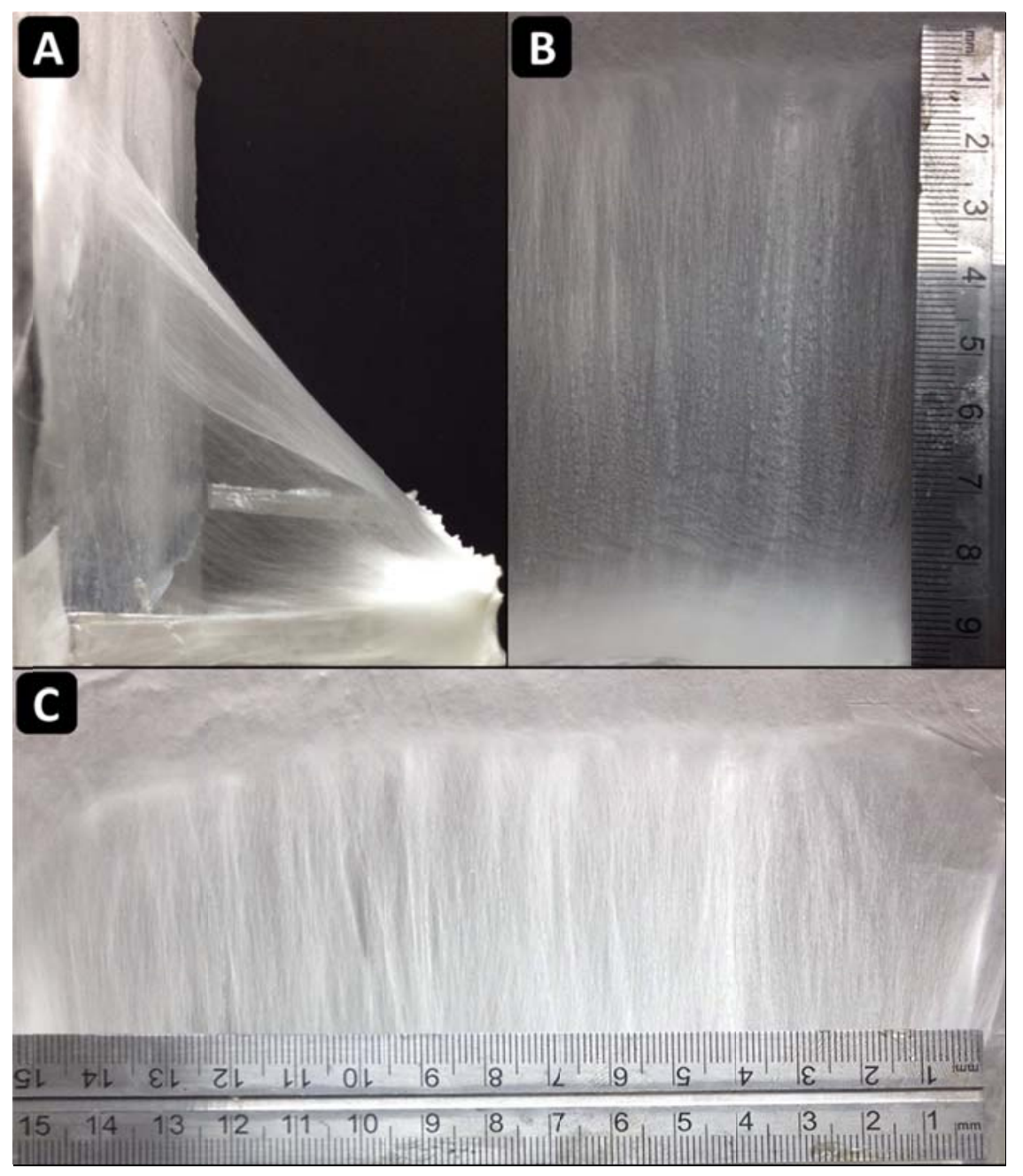

Figure 3.5. A newly designed collector for electrospinning. (A) An aluminum foil strip was horizontally attached to a vertical aluminum metal plate, it is clear that fibers were deposited and spanned between the aluminum foil strip and plate. (B) The picture shows the longest fibers could be collected from the newly designed collector. (C) The picture shows the width of the whole fiber mat obtained from the newly designed collector.
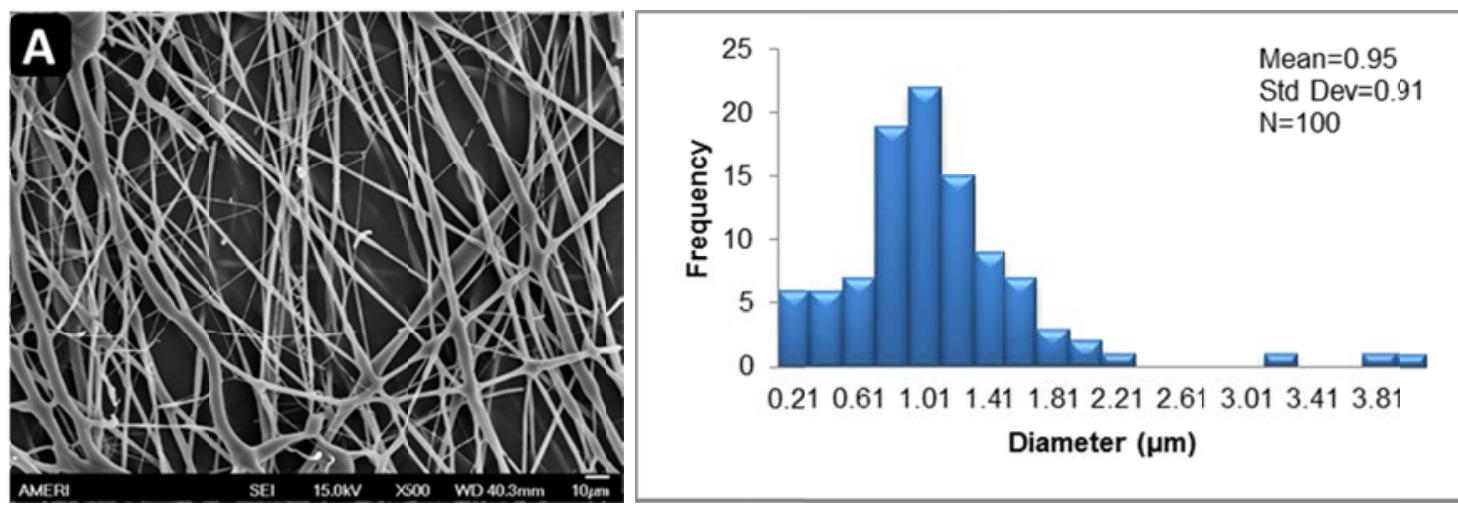

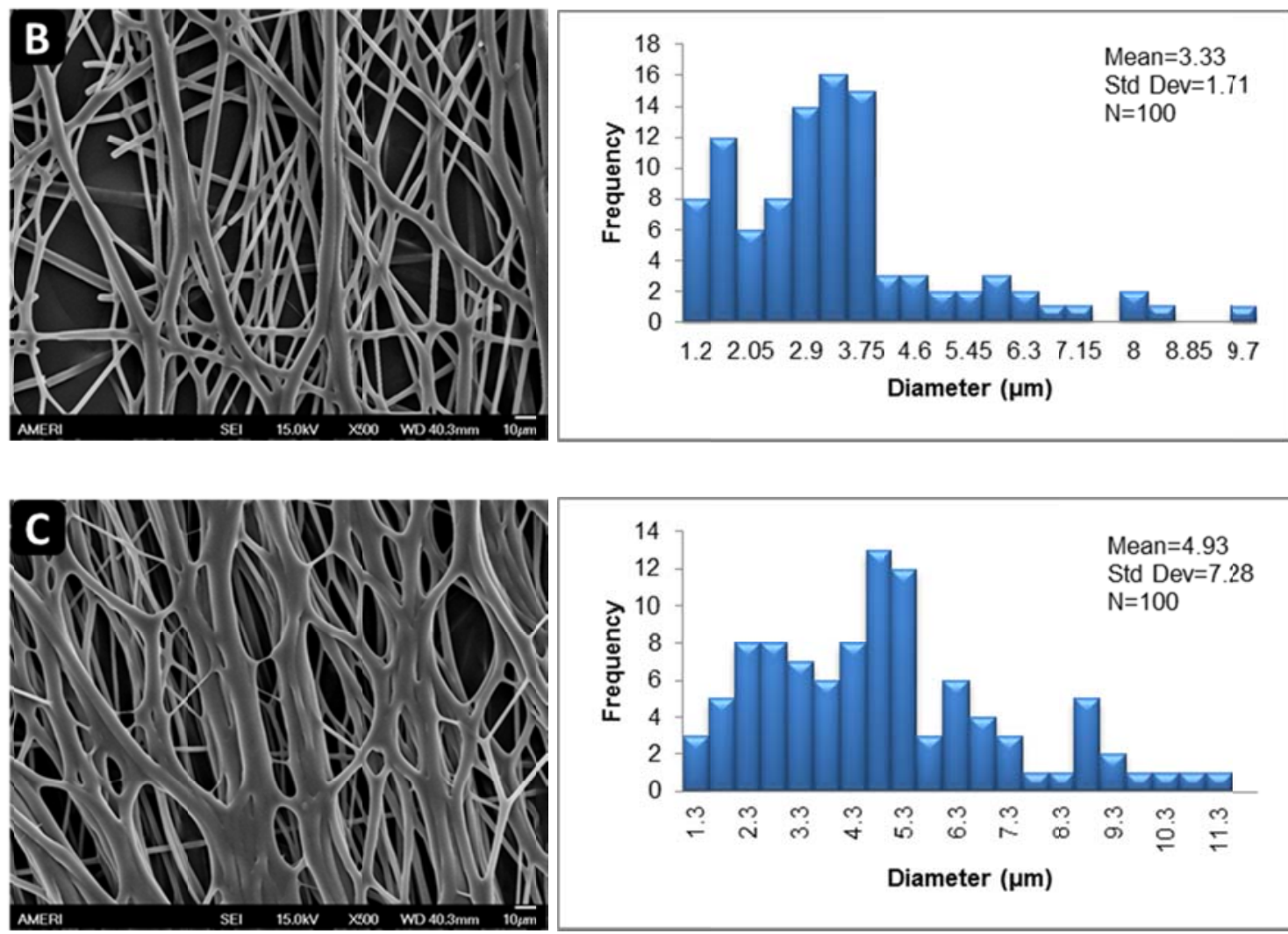

Figure 3.6. SEM images and diameter distribution of electrospun PGD fibers from (A) $30 \%$, (B) $40 \%$, and (C) $50 \%$ (w/w) polymeric solution (No fiber can be collected when the PGD concentration is lower than 30\%). The white scale bar represents $10 \mu \mathrm{m}$. The results of the Kruskal-Wallis Test and Mann-Whitney U-test showed that there is a significant difference between each 2 groups tested $(p<0.01)$. Diameters were measured using SEM pictures and 100 randomly selected areas were averaged.

\subsubsection{Raman spectroscopy}

Figure 3.7 shows the spectra of PGD/basal solution (BS) containing gelatin prior to and after electrospinning. Comparing these two spectra, slight differences of relative intensity were noted. This could be the result of the different board background of each spectrum, therefore no significant change is found in the chemical structure of the polymer solution during the electrospinning process. 


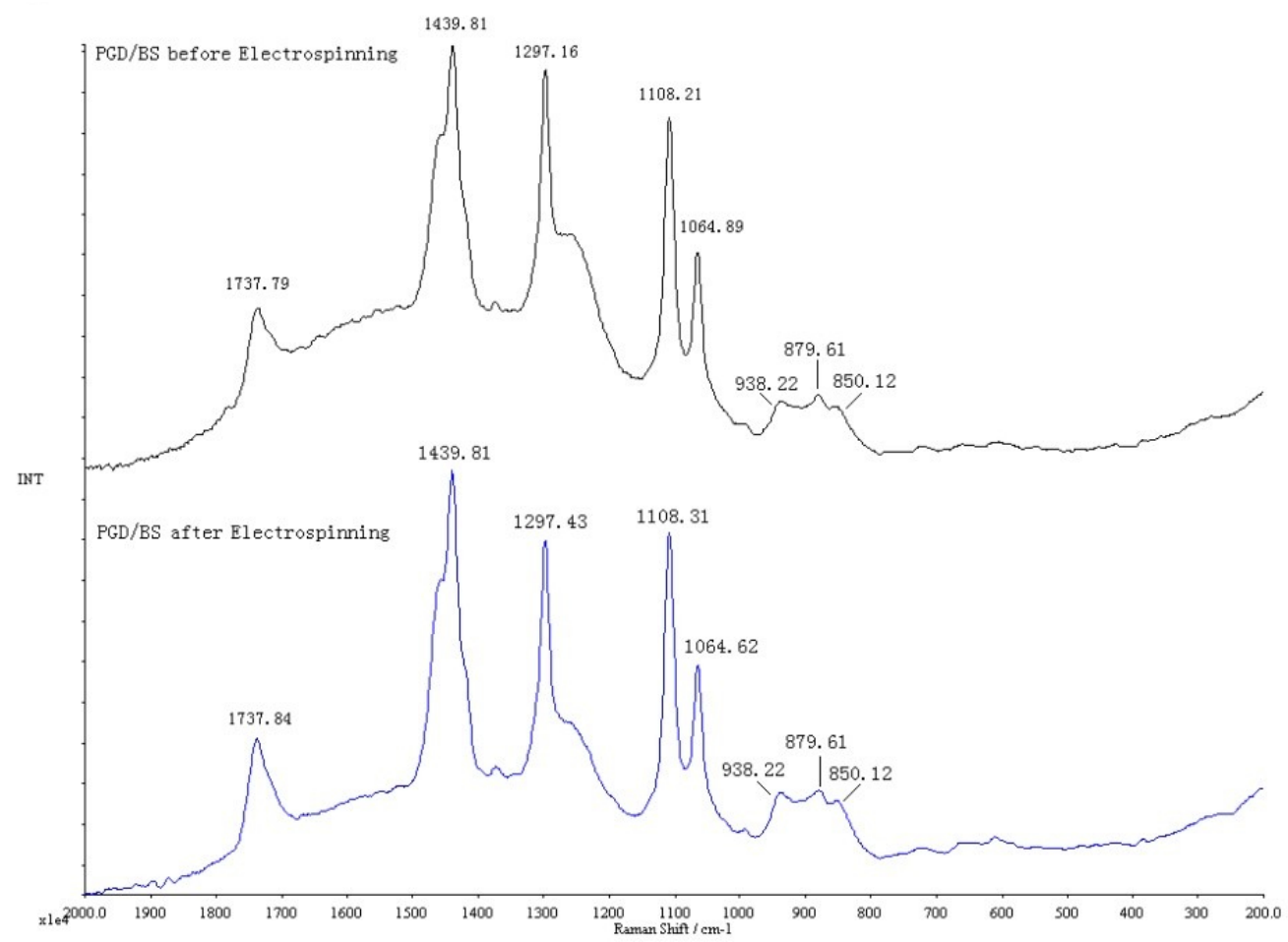

Figure 3.7. Raman spectra of PGD/BS fibers prior to and after electrospinning.

\subsubsection{Fiber dissolvability}

Dissolvability testing of the PGD with gelatin scaffolds was performed to study the stability of the fibers in PBS at $37{ }^{\circ} \mathrm{C}$ for four weeks. The fibers without crosslinking dissolved in PBS quickly (data not shown). In order to increase its stability, riboflavin, which is a photo initiator, was incorporated into the polymeric solution before electrospinning. Since ultraviolet (UV) light was used to sterilize the fibers, it is worth of investigating whether UV light could be, simultaneously, used for sterilization and crosslinking. After being exposed to UV light for 80 minutes, the fiber scaffolds maintained its structural integrity in PBS for a one month. Figure 3.8 shows the change in morphology and structure of the PGD with gelatin fibers during the degradation process. Compared to figure 3.6 , the 
morphology of some of the fibers changed after 4 days (Figure $3.8 \mathrm{~A}$ ) and this change became more obvious after two weeks, which is reflected by the fact that fibers were swelling and fused together (Figure 3.8B). On day 28, under higher magnification, even though some fibers were broken down into short pieces, the fiber structure was mostly preserved (Figure 3.8C).

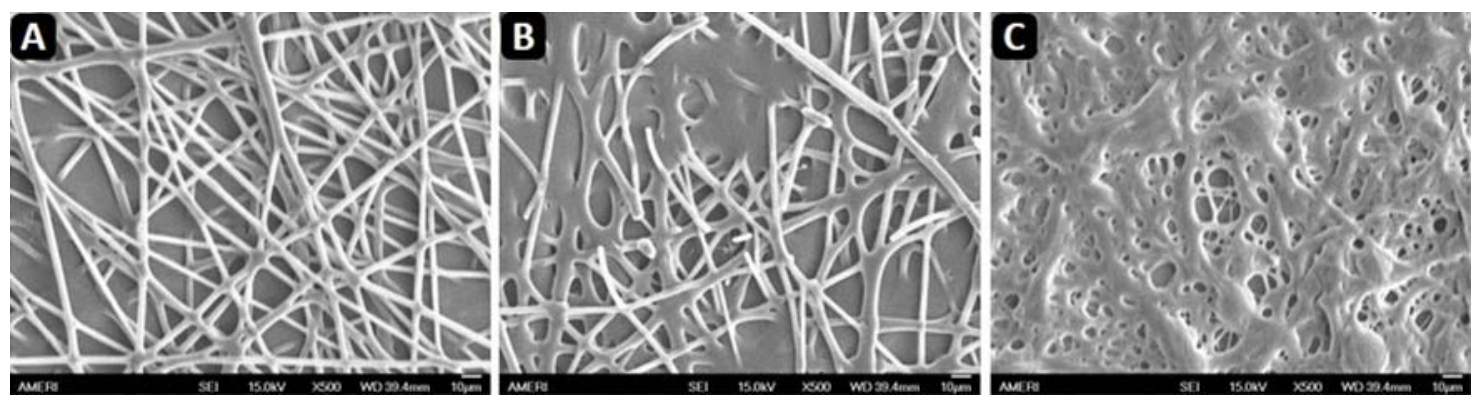

Figure 3.8. Surface morphology of the degraded PGD with gelatin fibers after different time points. PGD with gelatin fibers after (A) 4 days; (B) 14 days and (C) 28 days.

\subsubsection{In vitro studies of PGD/gelatin fiber scaffolds}

\subsubsection{Visualization of cells on fiber scaffolds}

Calcein AM, a cell-permeant dye, was used to visualize the live cells in PGD fibrous scaffolds. In live cells, the nonfluorescent calcein AM is converted to a green-fluorescent calcein after acetoxymethyl ester hydrolysis by intracellular esterases. After 1 day of cultivation of HFFs on fiber scaffolds in a 6-well low adherent plate as well as cultivation of the control group cells on the $35 \mathrm{~mm}$ tissue culture dish without fibers, Calcein AM dye was added and confocal microscopy was used to acquire the fluorescent images. The cell morphology on the two surfaces was considerably different. On the fiber scaffold, most cells attached to the fibers exhibited round shapes with less spreading, although a small fraction of cells developed multi-cell clusters (Figure 3.9A). In contrast, on 
the $35 \mathrm{~mm}$ tissue culture dish without fibers, the fibroblasts were well-spreading across the entire surface when observed under the confocal microscope (Figure $3.9 B)$.
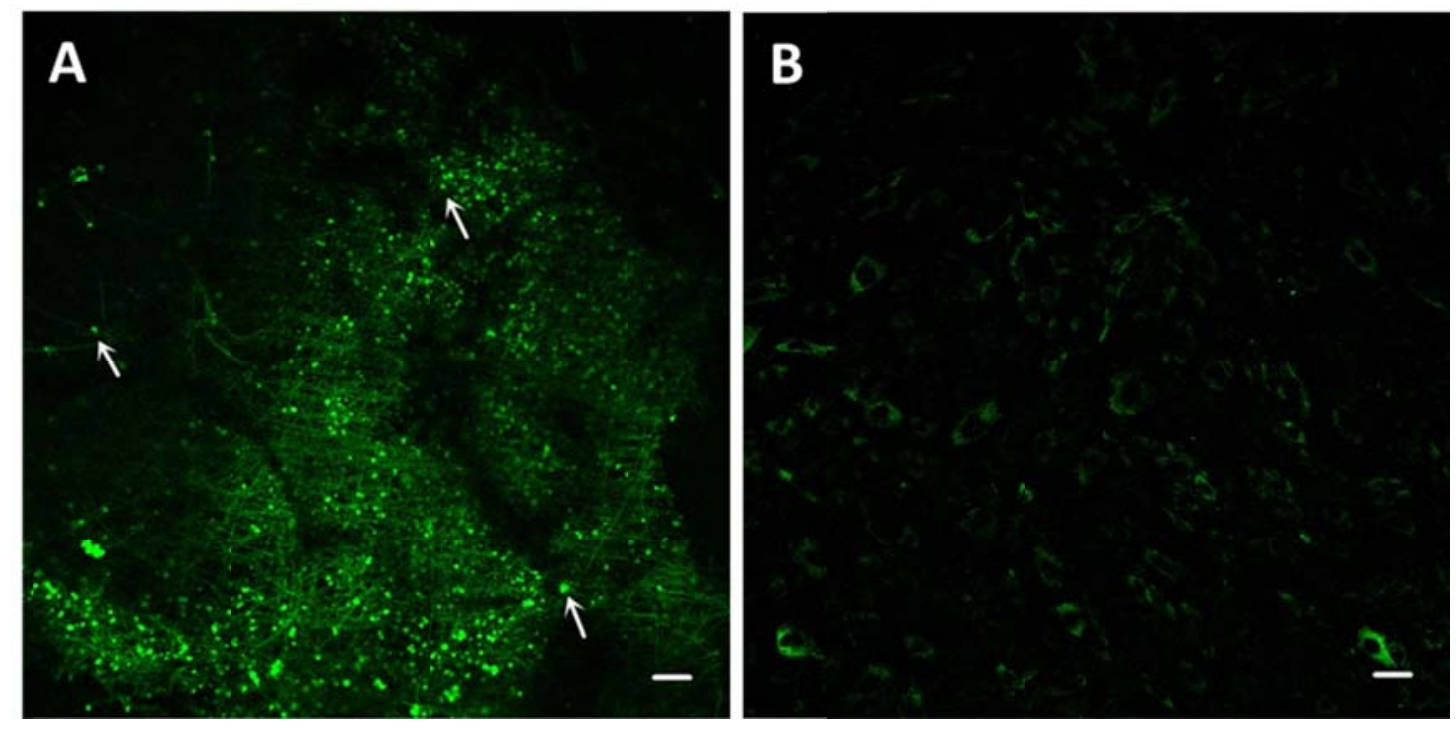

Figure 3.9. Cell adhesion on the PGD with gelatin fibers after 1 day culture. HFF cells staining with Calcein AM reagents (bright green, arrows) on the scaffold (A) and tissue culture dish (B). Scale bar: $50 \mu \mathrm{m}$.

\subsubsection{Cell viability}

The proliferation of the cells on the PGD fibers as well as the tissue culture dishes was compared by using Resazurin assay. This assay measured the metabolic activity of the cultured cells and the fluorescence intensity reading of the cell culture medium reflected the degree of viability. HFFs were seeded on PGD scaffolds with and without gelatin as well as tissue culture dishes at the cell density of $5.0 \times 10^{4}$ for up to 8 days. Figure 3.10 shows that the cells grown on the PGD with gelatin fibers had equivalent or better cell viability than the control group during the first 3 days. This could be due to the scaffolds providing more surface area for cell growth, when compared to the two dimensional tissue 
culture dishes. Cells on the PGD without gelatin fibers exhibited relatively lower proliferation compared to the other two groups. Overall, cells seeded on the PGD fibers with gelatin were comparable to cells grown in tissue culture dishes, but cells on the PGD fibers without gelatin continued to grow at a slow rate. This indicates that gelatin is an essential part of cell adhesion and survival on the fiber scaffolds.

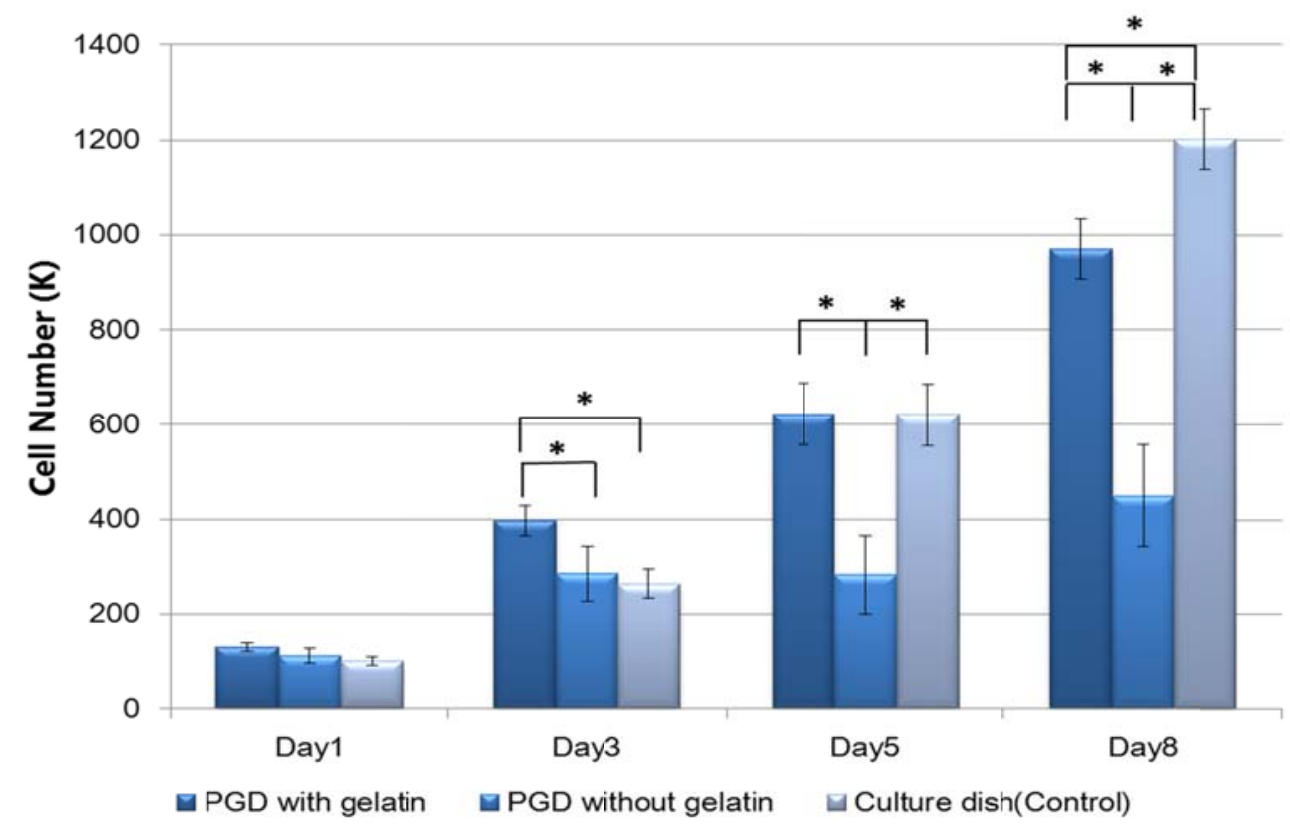

Figure 3.10. Resazurin assay showing the cell viability on PGD with Gelatin fibers and PGD without gelatin fibers after $1,3,5$, and 8 days. Cells directly cultured on the tissue culture dishes used as control. Sample number in each group, $n=4$. Average values and standard errors are from three replicate experiments. ${ }^{*} p<0.05$.

\subsection{Discussion}

Compared to conventional autografts and allografts, tissue engineering is an alternative strategy enabling tissues or organs in the body to be made de novo. It generally involves the use of cells cultured on scaffolds in combination with biological factors. Scaffolds in tissue engineering are required to meet 
certain minimum requirements for biochemical and physical properties, namely, mechanical stability, biocompatibility, and topography.

PGD is a biodegradable elastomeric polymer synthesized from glycerol and DDA. It is biocompatible, exhibits elastic behavior and unique shape memory [19]. The $M_{n}$ and $M_{w}$ of PGD after 4 days are approximately 20 and $111 \mathrm{kDa}$, respectively. The fibrous scaffolds made from PGD provide a good solution for soft tissue engineering because their elastic properties better match the mechanical properties of soft tissues when compared to other stiffer synthetic polymers. The properties of the electrospun fiber are greatly influenced by several processes and solution parameters. The composition of the polymeric solution is extremely important for achieving ideal fiber scaffolds. PGD concentration ranging from $30 \%$ to $50 \%(\mathrm{w} / \mathrm{w})$ with and without gelatin can generally produce the desired fiber mats. Increasing PGD concentration raises the viscosity of the polymer solution resulting in fibers with larger diameter size as well as a smaller porous structure. Although decreasing the PGD concentration will lessen the diameter of the fiber, there is a greater chance of having drops on the fiber mat.

With other synthetic polymers like PLGA and PCL for electrospinning PLGA/Gelatin/Elastin [172] and PCL/Gelatin fibers, more expensive and toxic solvents such as hexafluoro-2-proponol and trifluoroethanol are required to dissolve gelatin, elastin and polymers. In contrast, PGD can be easily dissolved in solvents like a mixture of ethanol and water, thus incorporation of watersoluble biomolecules such as gelatin into PGD in our study became a 
straightforward job. The advantages of using a less toxic solvent like ethanol include fabrication flexibility and minimizing the safety concern.

Furthermore, it is also interesting to monitor the chemical structure changes during the PGD polymerization and electrospinning fabrication. DDA is a molecule with carboxylic groups at both ends, and glycerol has three free hydroxyl groups. During the polymerization process, these two functional groups react to form a new ester bond after removing one water molecule. Glycerol with three hydroxyl groups allows the polymer to form branch structures and results in a three dimensional network after curing. The $\mathrm{C}=\mathrm{O}$ stretch, which appears at $1730-1740 \mathrm{~cm}^{-1}$ on raman spectra, was an indication of ester bonds and was present in the PGD polymer, PGD/Gelatin blend and the final electrospun fibers. The similarities in the Raman spectra of pre- and post-electrospun polymers demonstrated that the electrospinning process did not change the chemical structure of the polymer.

Crosslinking of electrospun fibers is carried out in an attempt to preserve the scaffold fibrous architecture. The fiber scaffolds before crosslinking dissolved quickly in PBS at $37{ }^{\circ} \mathrm{C}$ (Data not shown). Therefore, it is essential to stabilize the scaffold by using a crosslinking agent, which would preferably be nontoxic to the cells. Riboflavin, before known as vitamin B2 or additive E101, is an easily absorbed micronutrient with a key role in maintaining health in humans and animals [173]. According to the previous study, it also acts as a photo initiator to crosslink collagen with the help of short UV exposure for eye treatment [174, 175]. In this study, $0.1 \%$ riboflavin was incorporated into the polymer solution 
before electrospinning, and after the fiber mat was collected, it was exposed to UV light for around 80 minutes. Compared to the scaffold, which was not crosslinked, the crosslinked fiber mat could grossly maintain its structural integrity in PBS at $37^{\circ} \mathrm{C}$ for up to 28 days.

The continuous increase in cell numbers on the PGD with gelatin fiber scaffolds over a period of 8 days indicated that cell proliferation took place on these fiber mats, similar to the traditional 2 dimensional culture dish. In contrast, cells on the PGD without gelatin fibers exhibited relatively slower proliferation. This suggests that the natural polymer gelatin plays a key role in cell adhesion and proliferation. Both PGD and PEO are synthetic polymers; hence there are no binding sites for cells to attach to. Gelatin derived from collagen provides sites (ligands) for cell adhesion thus improving cell proliferation. Therefore, PGD blended with gelatin also exhibits similar properties for promoting cell adhesion and proliferation, which in turn enhances the fiber mat biocompatibility. 
CHAPTER 4 Modification of Electrospun Fiber Scaffolds Composed of PGDF for Neural Tissue Engineering.

\subsection{Introduction}

Biomaterials play a vital role in tissue engineering because it is the key ingredient of the scaffolds created to facilitate cell attachment, proliferation, differentiation, and, ultimately, new tissue formation. Many techniques have been developed for 3D scaffolds fabrication over the past 20 years. Recently, micro/nanofiber-based scaffolds produced via the electrospinning technique are gaining increasing interests since it offers the opportunity for control over the composition of the fibers along with porosity of the fiber meshes using a relatively simple experimental setup. Structurewise, electrospun fiber scaffolds bear a strong resemblance to the hierarchical structure of the extracellular matrix (ECM), an environment critical for cell survival, differentiation, signal transduction and nutrient/waste transport [13]. Furthermore, electrospun fibers can be modified using different physical or chemical methods to encapsulate or attach bioactive molecules such as native ECM proteins or RGD peptides to support and guide both the survival and differentiation of cells seeded on the scaffold. All these attributes make electrospun fibers a new class of promising scaffolds for neural tissue engineering.

\subsubsection{Embedding Biomolecules into fiber Scaffolds for Neural Tissue} Regeneration

While scaffold-based approaches have been utilized in the past to support the repair of neural tissue, a fundamental limitation of these strategies is the lack 
of appropriate biomolecules embedded in the scaffold. Without the presence of these biomolecules, continuous delivery requires a stream of complex surgical procedures to inject them into the site of injury [176]. Unfortunately, this does not ensure that the concentrations of these biomolecules remains constant over the duration of release.

Ever since the development of polymer-based implantable therapies, scientists have worked hard to learn how to further functionalize these surfaces in order to create specialized cell surface interactions. Two fundamental methods exist for coating polymers - using proteins and using peptides [177]. Adhesive proteins such as fibronectin, collagen and laminin were initially considered and tested, but they are often purified from other organisms and hence may create an immune response. In addition, due to their large size, it is not certain if their orientation at any particular moment will be suitable for cell adhesion. Finally, they are potential targets of proteolytic degradation; therefore, their concentration will not be constant over time. On the other hand, peptides have emerged as a superior option due to their enhanced stability and higher potential packing density. While protein-based options originating as ECM components have the potential to activate a variety of cell recognition motifs due to their size, peptides are far more selective and can activate single motifs. Therefore the RGD-sequence and laminin were chosen as the biomolecular peptides of this study.

4.1.2 Functions of poly (glycerol- dodecanedioate co-fumarate) 
PGDF is a novel polymer derived from previously researched polymer PGD [143]. PGD was chosen as a base for the polymer since its mechanical properties are suitable for soft tissue engineering. PGD is advantageous since its synthesis is simple, non-toxic and has good biocompatibility. In addition, the diameter of derived fibers can be adjusted easily by changing the concentration of PGD.

One disadvantage of the PGD polymer is that it is composed of saturated polyester chains that only have hydroxyl or carboxylic groups available for chemical modification. If another double-bond containing monomer is introduced, the resulting polymers with unsaturated polyester chains may provide new modification possibilities. These properties, in turn, can be used to modify PGD fibers and facilitate cell attachments. For this reason, Fumaric acid, a non-toxic, naturally occurring monomer, was incorporated into the PGD polymer synthesis process and produced a PGD derivative referred as poly (glycerol dodecanedioate co-fumarate) (PGDF). Fumaric acid has also been demonstrated to be both neuroprotective and cardioprotective through activation of the Nrf2 antioxidant pathway $[127,128]$. Sodium Fumarate (NaF) is derived from Fumaric Acid, which is a metabolite and intermediate product of the citric acid cycle [129]. Time-dependent and concentration-dependent studies have been carried out that demonstrate $\mathrm{NaF}$ to be immunomodulatory and able to reduce oxidative stress without altering fundamental network activity. In vitro, its addition has been shown to improve neuronal survival [178]. In vivo, fumarate works to better preserve the integrity of myelin, axons and neurons. The choice to add it to the 
experimental protocol was due to a fundamental belief that its preservative ability would impact cell survival and induction efficiency.

\subsubsection{Peptides modification on the synthetic polymers}

The use of synthetic polymers modified with peptides containing RGD (the cell recognition motif) not only triggers cell adhesion but can be used to selectively modify the activity of certain cell lines [177]. The RGD peptide sequence is useful for stimulating adhesion through the activation of integrinfamily cell adhesion receptors. Integrins are not only used for physical anchoring but also promote signal transduction through the membrane of the cell.

Laminin was chosen as a biomolecule to integrate into the scaffold since it is a naturally occurring ECM protein that promotes the formation of neurites [179]. It provides a local environment that mimics neural tissue and can be added using covalent binding or physical adsorption. Laminin is continuously synthesized after nerve injury and plays essential roles in cell migration, differentiation and the growth and elongation of axons. Myelinating phenotypes of Schwann cells cannot occur without the presence of laminin. Laminin has been used previously in electrospinning, for example, incorporated into electrospun poly(L-lactic acid)co-poly(e-caprolactone) (PLCL) [180]. This research is the first documented use of laminin on the electrospun PGDF scaffold and based on the properties of laminin there is a reason to believe this choice is essential to ensure neural growth and survival. 


\subsection{Experimental}

\subsubsection{PGDF fiber design and fabrication}

PGDF polymer was made from glycerol, DDA and Fumaric acid (FA) (St Louis, MO) (Figure 4.1A). To begin with, glycerol and DDA were mixed in beaker 1 in a molar ratio of 0.6:0:2 and glycerol plus FA in beaker 2 in a molar ratio of 0.4:0.8. These two beakers were kept at $120^{\circ} \mathrm{C}$ for 72 hours until both the acids have been completely reacted with glycerol. Then the pre-polymers in both beakers were mixed together and kept at the same temperature for another 24 hours to procure PGDF polymer. For basal solution (BS) preparation, a mixture of poly (ethylene oxide) (PEO), gelatin, and solvent (65\% ethanol and $35 \%$ water) with a combination of $1.5: 3: 95.5(\mathrm{w} / \mathrm{w})$, was heated in a low temperature oven (around $65^{\circ} \mathrm{C}$ ) with stirring every 10 minutes until it became a homogeneous solution. The testing solutions for fabricating electrospun fibers were composed of $60 \%$ polymer (PGDF:PGD=5:5) and $40 \% \mathrm{BS}, 50 \%$ polymer and $50 \% \mathrm{BS}, 40 \%$ polymer and $60 \% \mathrm{BS}$, and $30 \%$ polymer and $70 \% \mathrm{BS}$, respectively. In addition, $0.1 \%$ riboflavin was incorporated into the polymer solution as a photoinitiator for later crosslinking and peptides immobilization process. These polymeric solution were then underwent the electrospinning process. 
A

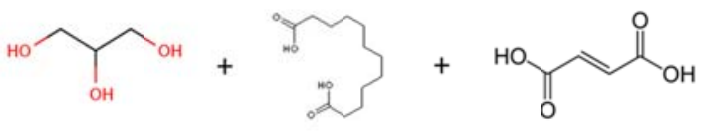

Glycerol

DDA

FA

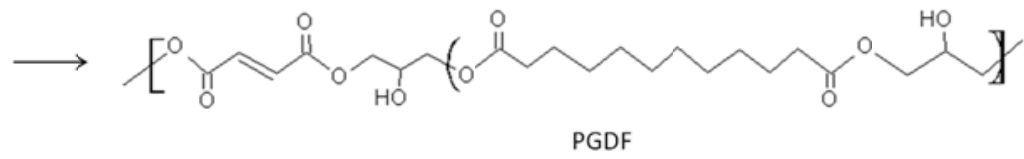

B

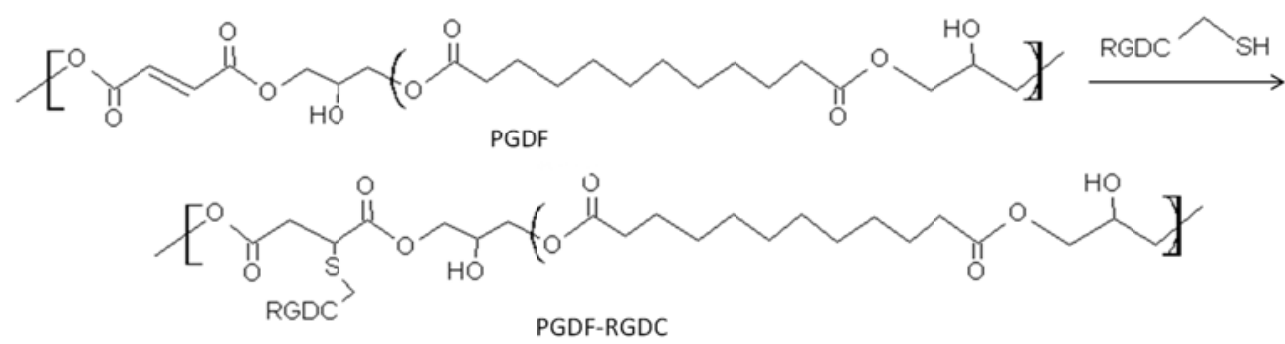

Figure 4.1. (A) Synthetic biodegradable elastomer, poly (glycerol-dodecanedioate co-fumarate). (B) RGDC immobilization.

PGDF fiber scaffolds were fabricated as previously described [143]. Briefly, polymer solution was fed into an $8 \mathrm{ml}$ standard syringe and delivered through an $18 \mathrm{G}$ blunted stainless steel needle by a syringe pump (Fish scientific, USA) at a flow rate of $0.5 \mathrm{ml} / \mathrm{h}$. The operating voltage was $14.6 \mathrm{kV}$ (SL30, Spellman High Voltage Electronics Corporation, USA) and the distance range between the needle tip and the ground collector was $18-25 \mathrm{~cm}$ depending on the different concentration of the polymer solution. An aluminum foil strip was attached perpendicular to a flat metal plate as the electrospinning collector, and fibers formed across the gap between this strip and the plate. The syringe pump was placed at an angle of 15 degrees with the horizontal to prevent aggregation of the fibers at the front of the strip. Fiber mats were exposed to UV light for 2 hours to enhance the crosslinking process. 


\subsubsection{Characterization of PGDF fiber scaffolds}

\subsubsection{SEM}

The electrospun fibers of PGDF in different concentration were analyzed using Scanning Electron Microscopy (SEM, JEOL JSM6330F, Tokyo, Japan) at various magnifications. Before imaging, the fiber samples were placed in an Auto sputter coater (PELCO SC-7) for 50 sec to deposit a thin Au conductive film. The average diameters of the fibers were determined by means of Image $\mathrm{J} 1.47 \mathrm{v}$ software at 100 different locations of the 500X SEM images.

\subsubsection{Dissolvability test}

After 2 hours UV exposures, electrospun fiber mats were cut into round pieces of the same size and placed into 24-well plate for dissolvability test. One milliliters of the Phosphate Buffered Saline (PBS) were added to each well and the plate was kept at $37{ }^{\circ} \mathrm{C}$ for up to 2 month to test the dissolvability of the scaffolds.

\subsubsection{FA Release Behaviors of Electrospun fiber}

The electrospun fiber mats were sectioned into round pieces and each sample was incubated into $10 \mathrm{~mL}$ of PBS at $37^{\circ} \mathrm{C}$. Samples of $1 \mathrm{~mL}$ released solution were taken from the incubation solution at $3 \mathrm{~h}, 24 \mathrm{~h}, 72 \mathrm{~h}, 96 \mathrm{~h}, 150 \mathrm{~h}$, 168 h, 192 h, 216 h, 240 h, 264 h, 336 h and 360 h after incubation, while an

equal amount of fresh PBS was added back to the dissolution medium. The amount of FA was detected with a UV spectrophotometer (Synergy HT, BioTek) at a maximal absorption peak of $230 \mathrm{~nm}$. For standard samples with a 
concentration from $15 \mu \mathrm{M}$ to $1000 \mu \mathrm{M}$ was determined between the absorption strength and FA concentration.

\subsubsection{Raman spectroscopy}

Raman spectra were taken with Raman Micro 400 Series Raman Spectroscopy (Perkin Elmer) using a 50X objective lens and with a 2-second exposure time. These spectrum data were used to evaluate the polymerization process and the chemical structures of PGD and PGDF. The samples were taped to the shiny side of an aluminum foil covering the microscope slides[167].

\subsubsection{Peptides Immobilization}

The fiber mats were first cut into round pieces and placed into 24-well plate. They were then incubated with RGDC peptides or Laminin peptides or PBS as a control and exposed to blue light for 3 hours. Thereafter, the samples were kept in incubator at $37^{\circ} \mathrm{C}$ for another hour and then washed with PBS twice to get rid of the unbonded peptides. Fiber samples treated in different groups were dissolved in $95 \%$ ethanol. Then the dissolution medium were transferred to UV-Cuvette (BRAND, Sigma-Aldrich) and read with UV spectrophotometer.

4.2.3 In vitro studies of PGDF fibers with mouse embryonic stem cells

\subsubsection{Cell seeding and distribution}

Fiber samples made from different PGDF concentration were cut into small round pieces of the same size and placed into a 24-well. For pre-seeding treatment, specimens were soaked in peptides or PBS (as control) for $3 \mathrm{~h}$ under blue light and subsequently immersed in fresh medium and kept at $37{ }^{\circ} \mathrm{C}$ overnight. Before seeding, cells were counted using a hemocytometer and then 
seeded to the scaffolds at a $5.0 \times 10^{4}$ cells/well density. Following the addition of the cell suspension droplet, the fiber mats were kept in an incubator at $37^{\circ} \mathrm{C}$ and $5 \% \mathrm{CO}_{2}$ to allow cell attachment.

\subsubsection{Cell attachment and survival}

Resazurin fluorescence dye (Sigma-Aldrich, St Louis, MO), which had been shown to be identical to the commercial assay Alamar Blue [141], were applied as an index of cellular viability [168]. The resazurin reagent was mixed with fresh medium in 1:10 (v/v) and added to each well. Cell culture plate was kept in an incubator at $37{ }^{\circ} \mathrm{C}$ and $5 \% \mathrm{CO}_{2}$ for $4 \mathrm{~h}$ to allow the cells to metabolize the resazurin. Then $100 \mu \mathrm{l}$ resazurin/medium mixture of different well were collected into a 96-well plate in triplicate. Then the 96-well plate reader was set to measure the fluorescence intensity of a $590 \mathrm{~nm}$ emission with an excitation wavelength of $530 \mathrm{~nm}$. The same procedure was repeated at different time points (day4, day6, day8 and day11).

\subsubsection{Phenotype expression}

Phenotype expression of the cells on PGDF fiber was examined through real-time PCR. Total RNA was prepared from fiber cell culture sample on Day 11 using SV Total RNA Isolation System (Promega). Each mRNA preparations was reverse transcribed into cDNA using olig(dT $)^{15}$, as described by the manufacturer (GoScript Reverse Transcription System, Promega). Real-time PCR was performed on a Real Time PCR System (Applied Biosystems). For amplification,

the cycling parameters consist of $1 \mathrm{cycle}$ at $95^{\circ} \mathrm{C}$ for 2 min and $20 \mathrm{~s}$, followed by 40 cycles of $95{ }^{\circ} \mathrm{C}$ for $3 \mathrm{~s}$ and $60^{\circ} \mathrm{C}$ for $1 \mathrm{~min}$. The comparative CT method was 
utilized to calculate the relative expression of each target gene, and each is normalized relative to the house keeping gene expression of glyceraldehydes-3phosphate dehydrogenase (GAPDH) mRNA.

\subsubsection{Statistics}

Kruskal-Wallis Test and Mann-Whitney U-test were performed to determine the statistical difference between groups in fiber diameter distribution. In both cases, the statistical significance was set at $p<0.01$. Analysis of variance (ANOVA) and Turkey's HSD tests were used to evaluate the significance between the PGDF-RGDC, PGDF-Laminin and PGDF group (Sample number in each group: $n=3$, experiment was repeated 3 times) for peptides binding and cell viability testing.

\subsection{Results}

\subsubsection{Characterization of PGDF electrospun fibers}

\subsubsection{Fabrication of scaffolds with different fiber diameter}

Electrospun fiber scaffolds were examined by using SEM to determine the morphology and diameter of the fibers. By varying the concentration of PGDF $(30 \%, 40 \%, 50 \%, 60 \%)$, electrospun fibers of various diameters could be generated (Figure 4.2 A-D). A histogram of the fiber diameters for each polymer concentration was conducted to examine the fiber diameter distribution (Figure 4.2 E-H). Diameters were measured using SEM pictures and 100 randomly selected areas were averaged. A significant difference was seen in the mean fiber diameter of $30 \%(1.756 \pm 0.813 \mu \mathrm{m}), 40 \%(2.597 \pm 0.765 \mu \mathrm{m}), 50 \%(3.697$ $\pm 0.807 \mu \mathrm{m})$ and $60 \%(4.925 \pm 2.148 \mu \mathrm{m})$. It is evident that the spread of fiber 
diameters remains small until the PGDF concentration reaches $60 \%$ and that the concentration of the PGDF controls the diameter of the generated fiber matrices. The results of the Kruskal-Wallis test and Mann-Whitney U-test showed a significant difference between each possible pair of groups within the four options $(p<0.001)$.

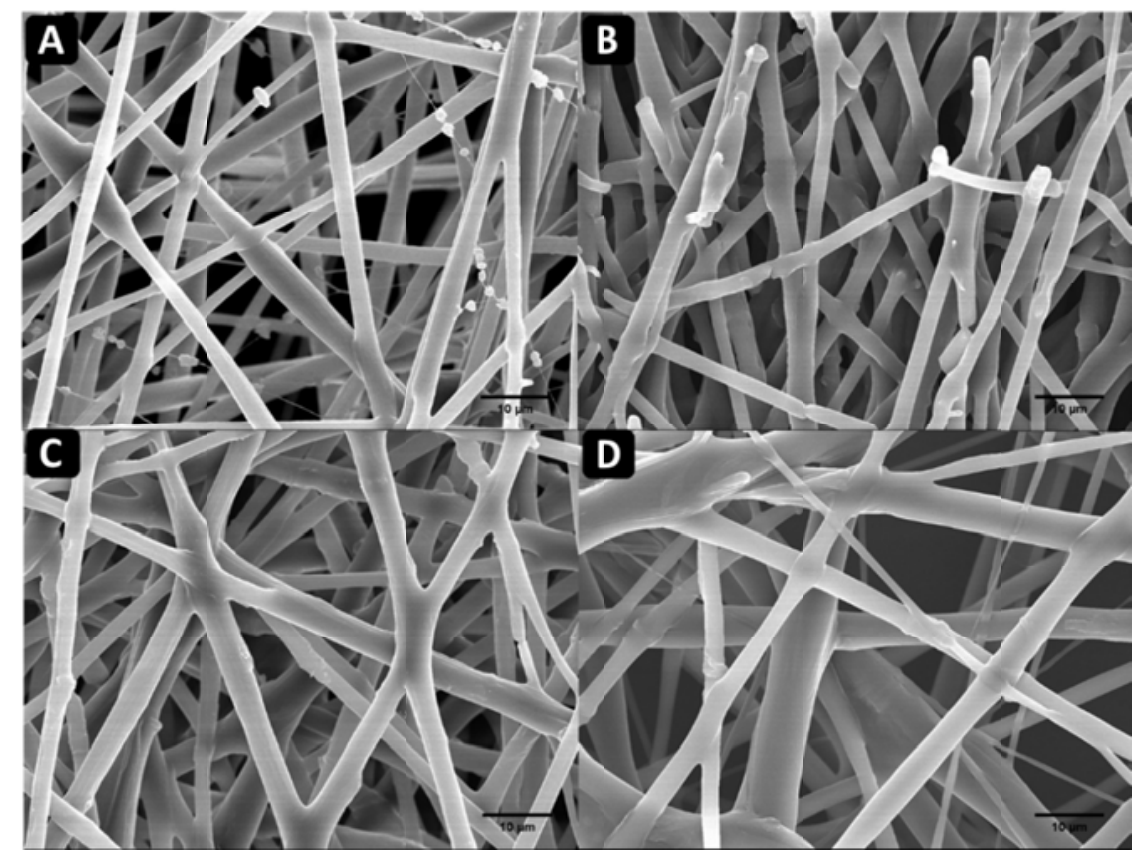



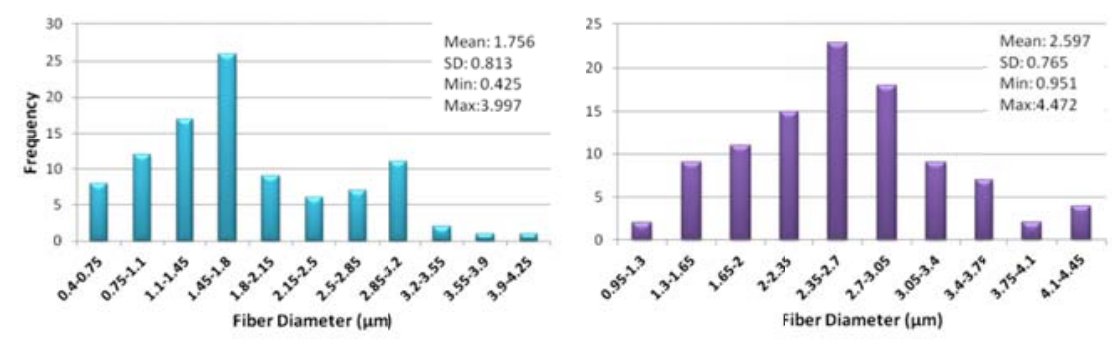

G

H
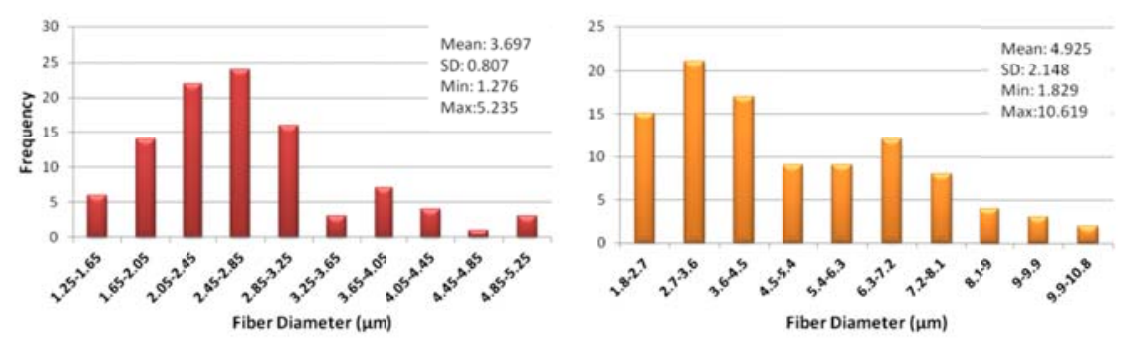

Figure 4.2. SEM images (A-D) and diameter distribution (E-H) of electrospun PGDF fibers from $(A, E) 30 \%,(B, F) 40 \%$, and $(C, G) 50 \%$ and $(D, H) 60 \%(w / w)$ polymeric solution. Scale bar: $10 \mu \mathrm{m}$. The results of the Kruskal-Wallis test and Mann-Whitney U-test showed that there is a significant difference between each possible pair of two groups $(p<0.001)$. Diameters were measured using SEM pictures and 100 randomly selected areas were averaged.

\subsubsection{Electrospun fiber dissolvability}

Dissolvability test of the PGDF fiber scaffolds was performed to study the stability of the fibers in PBS at $37^{\circ} \mathrm{C}$ for up to 2 months (Figure 4.3). This test is important since an ultimate goal of this fiber scaffold is implantation in the body for injury repair. By validating the stability of these fibers, it could help to understand the viability of these scaffolds for long-term therapy. As mentioned in the previous paper [143], a photo initiator called riboflavin was incorporated into the polymer solution before electrospinning. Figure 4.3 shows the change in structure of the PGDF fibers during the degradation. There was no obvious 
morphology change during the first month. Even though fibers were broken down into short pieces after 2 months, the fiber structure was mostly preserved.

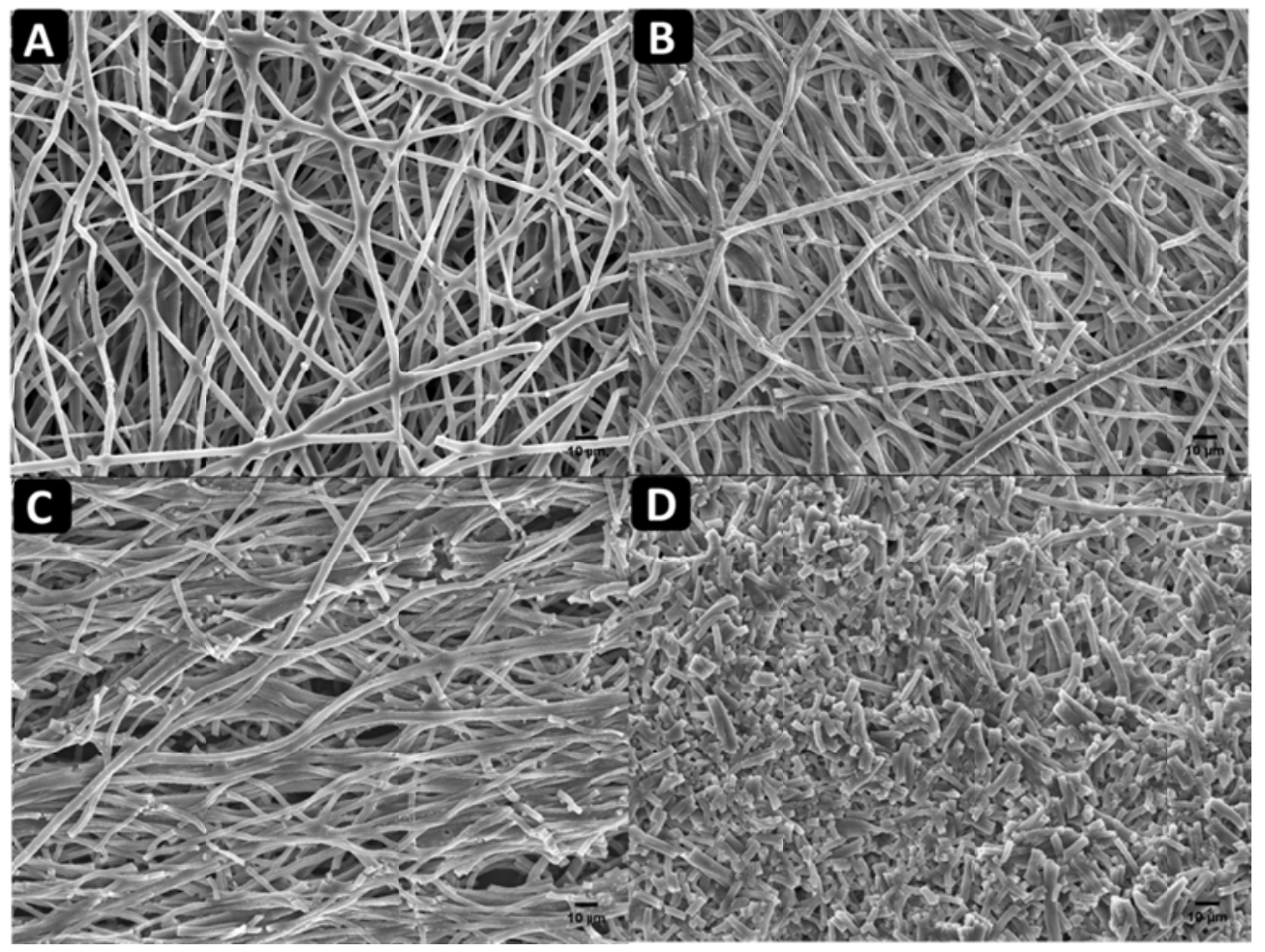

Figure 4.3. Surface morphology of PGDF fibers on (A) Day 1, (B) Day 14, (C) Day 32 and (D) Day 64. UV crosslinking was used to preserve the overall architecture in order to prevent rapid degradation.

\subsubsection{Fumaric acid release profile from electrospun fibers}

PGDF is a biodegradable polymer and thus it is also valuable to understand how quickly FA is released into the surrounding tissue. Using a UV spectrophotometer, FA can be detected at a wavelength of $230 \mathrm{~nm}$. The FA cumulative releasing profile of PGDF fibers with different concentration is shown in Figure 4.4. Sustained release of FA from PGDF fibers was obtained for 15 days, with little change in the rate by which it was released. From Figure 4.3 , it is not difficult to notice that fiber morphology remained relatively unchanged during 
the first month, with the most significant changes occurring between month one and two. The maintenance of fiber morphology over this first month is likely the primary cause of the linearity of the FA release profile over this time period. These results suggest that diffusion processes from the fiber surface are a stronger mechanism of FA release than bulk flow through degradation of the fiber scaffold.

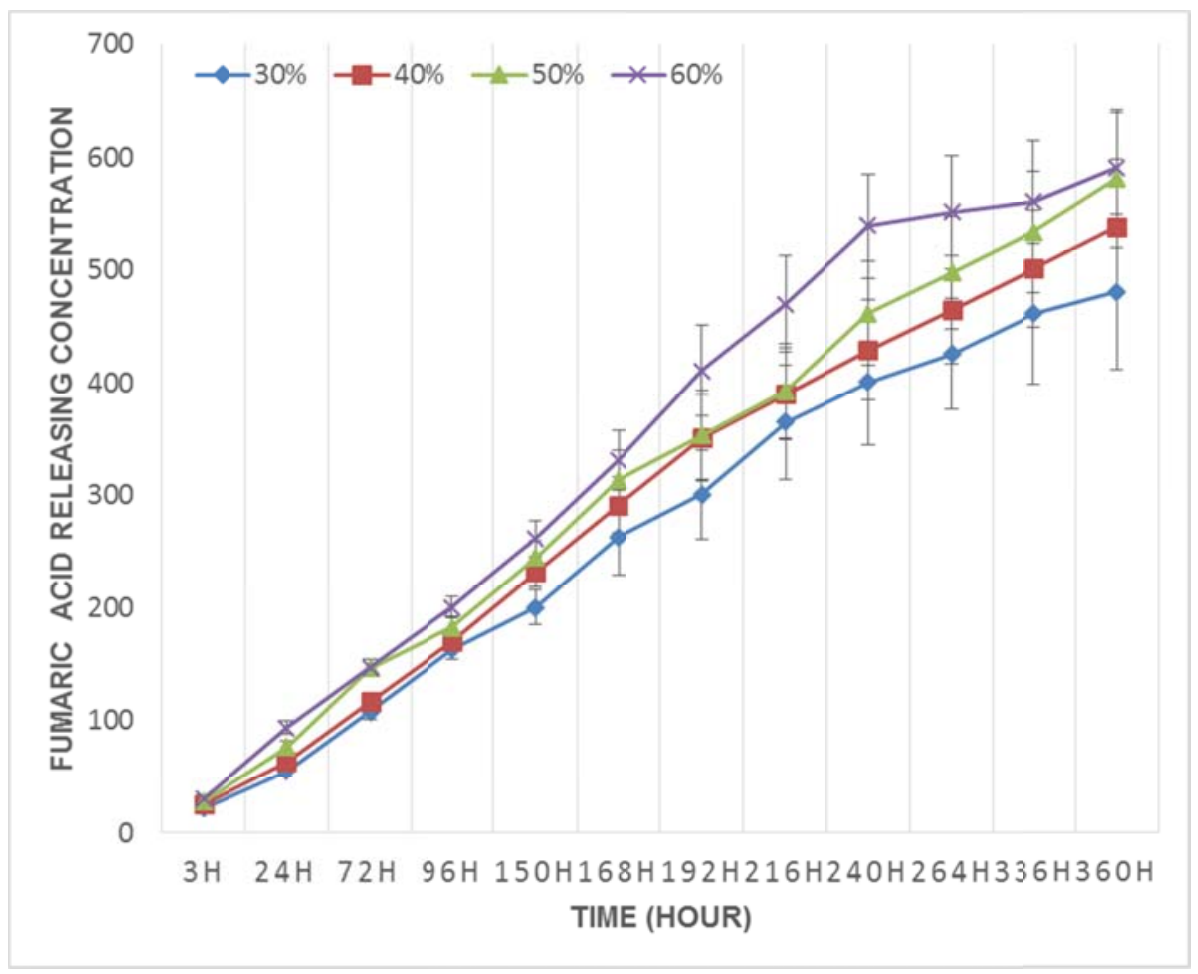

Figure 4.4. Releasing profile of FA from PGDF electrospun fibers. Concentration unit: $\mu \mathrm{M}$. Sample number in each group: $n=3$, experiment was repeated 3 times

\subsubsection{Raman spectroscopy}

Raman spectroscopy was used to investigate the chemical structure of PGDF and PGD polymer. Figure 4.5 shows the spectra of these 2 polymers, respectively. During the polymer synthesis, the carboxylic group in DDA $(-\mathrm{COOH}$, 
$\left.1644.60 \mathrm{~cm}^{-1}\right)$ reacted with hydroxyl groups in glycerol $\left(-\mathrm{OH}, 1251.13 \mathrm{~cm}^{-1}\right)$ to form a new ester bond $\left(\mathrm{C}=\mathrm{O}\right.$, around $\left.1735 \mathrm{~cm}^{-1}\right)$ after removing one water molecule. This is demonstrated in the spectrum of both polymers. Other bands around $1297 \mathrm{~cm}^{-1}$ and $1438 \mathrm{~cm}^{-1}$ are due to $\mathrm{C}-\mathrm{C}$ stretching vibrations, and $\mathrm{CH}_{2}$ twist vibrations and deformations, respectively. The only structure difference between $P G D F$ and $P G D$ is at band $1665 \mathrm{~cm}^{-1}$, which represents the $C=C$ introduced from FA. This double bond will break and react with the Thiol group in RGDC or laminin peptide, which is called Michael addition.

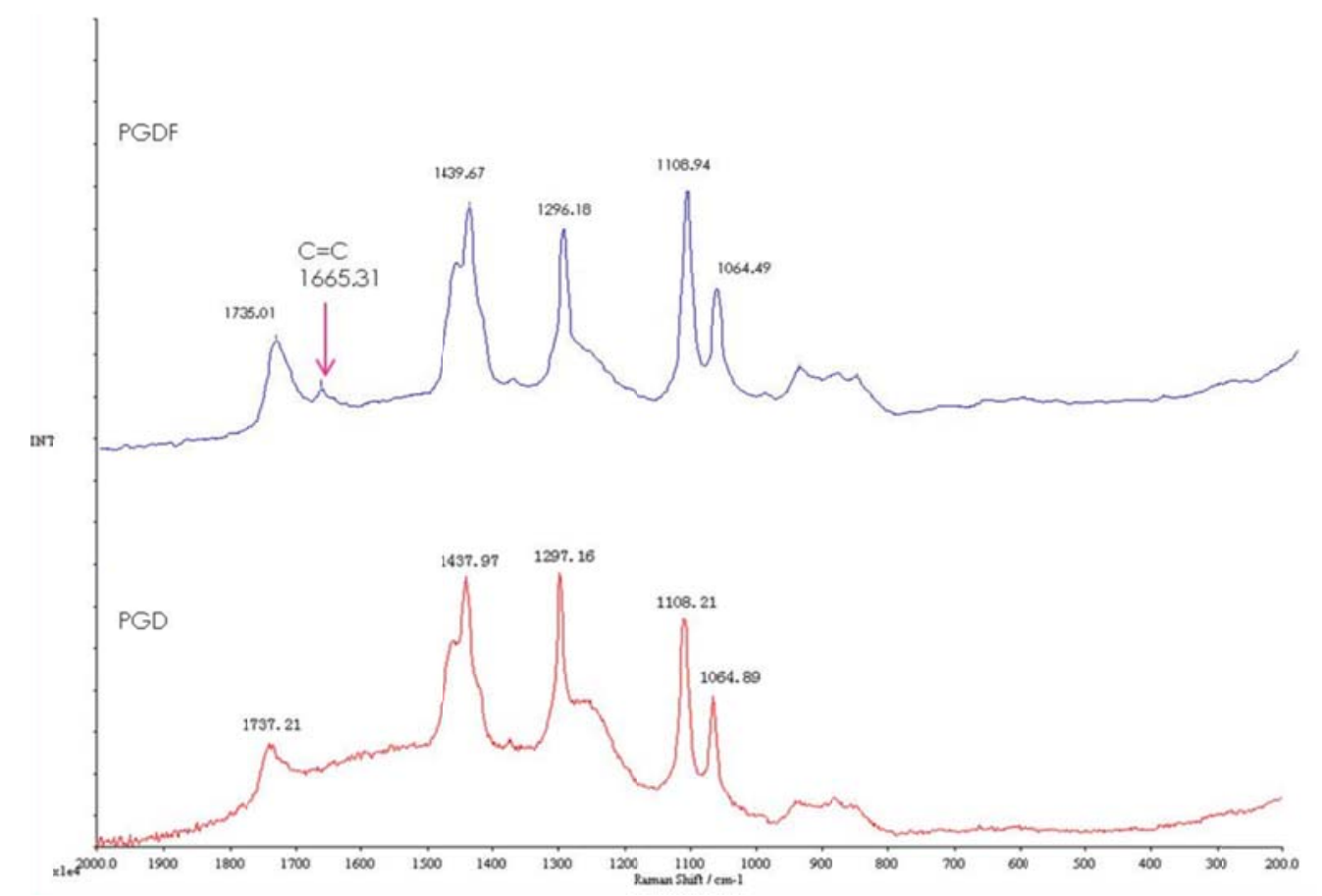

Figure 4.5. Raman spectra of PGDF and PGD, respectively. Double bond from FA was brought into the polymer for functional modification purpose. 


\subsubsection{Effect of peptides immobilized fibers on cell viability}

\subsubsection{UV detection of peptides binding}

Using UV spectroscopy, it is simple to detect the presence of the double bond in FA. Since the RGDC and laminin peptides break this double bond when bound to the polymer, there should be a significant difference in the double bond concentration with and without the peptides (Figure 4.6). To determine the $\mu \mathrm{M}$ concentration of double bond from the absorbance value of the spectrophotometer, a standardized curve was developed using different concentrations of FA. As a control, the fiber was incubated with PBS alone as compared to the experimental groups where it was incubated with the RGDC and Laminin peptides. In the control group, regardless of the polymer concentration, a higher concentration of the double bond FA was detected and expected since it would be retained, instead of being broken to bind the peptides as in the two experimental groups. 


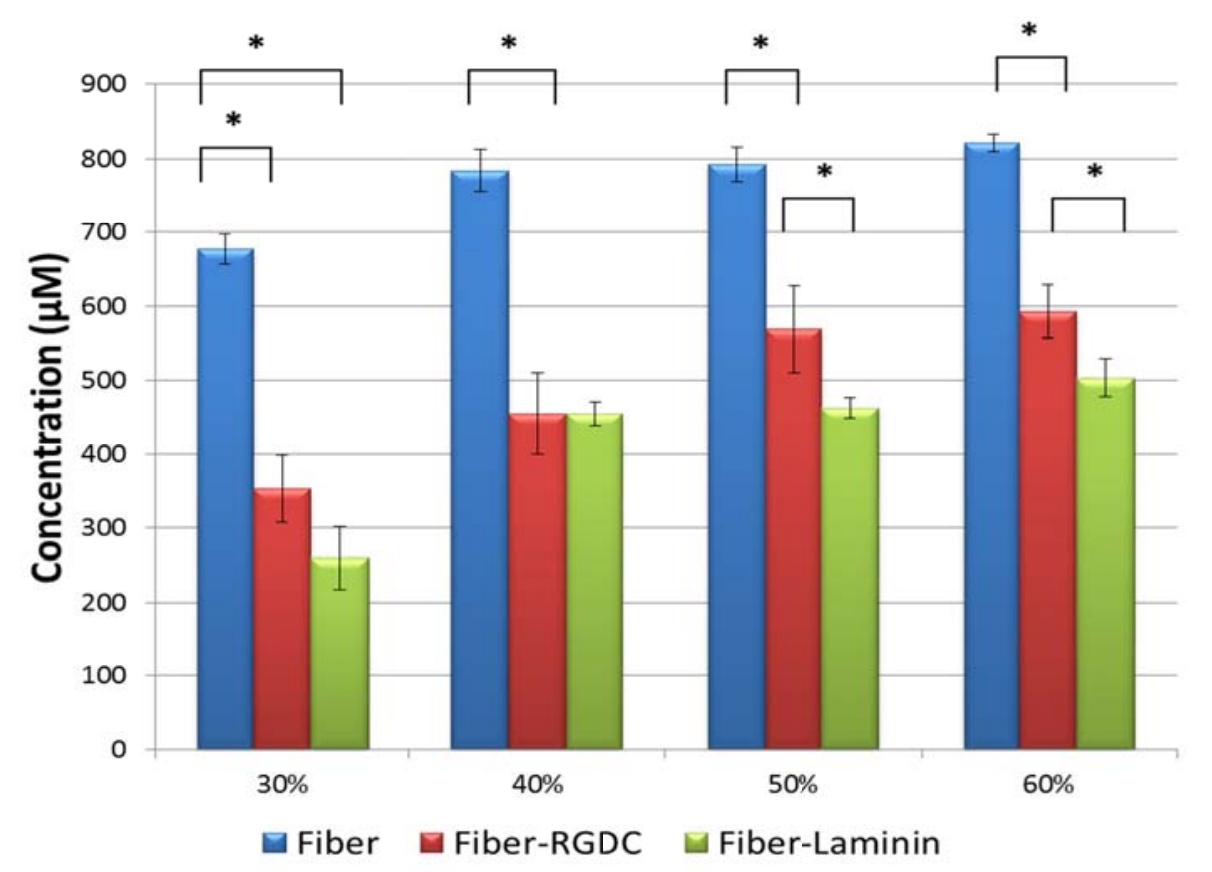

Figure 4.6. Concentration of double bonded in PGDF fiber with and without presence of peptide groups. The binding of each peptide group was measured individually using UV spectrophotometry to ensure that it broke the double bond to bind to the PGDF. Significant differences are indicated in the figure with an asterisk. Sample number in each group: $n=3$, experiment was repeated 2 times $(p<0.01)$.

\subsubsection{Cell viability within the scaffold}

Cell viability on PGDF and PGDF-modified fibers was evaluated by using Resazurin assay, which measures the metabolic activity of the cultured cells based on the fluorescent intensity reading. Mouse ESCs were seeded on the fiber mats at a cell density of $5.0 \times 10^{4}$ for 11 days. Figure 4.7 shows the cell viability of PGDF and peptide-modified PGDF result as well as the confocal microscopy images. While mESCs were plated on day 0 , by day 4 they had begun differentiating into neural stem cells. These cells grown on the fiber were far more viable with the peptides than without them. Although there was not a significant difference between viability using RGDC versus Laminin as the 
peptide of choice, there was a very significant difference between peptide and non-peptide polymer matrices. These results are especially important on day 11 , when the cells became mature motor neurons. Results from confocal microscopy clearly show a strong difference in cell viability between these three groups (Figure 4.7 B-D).

A
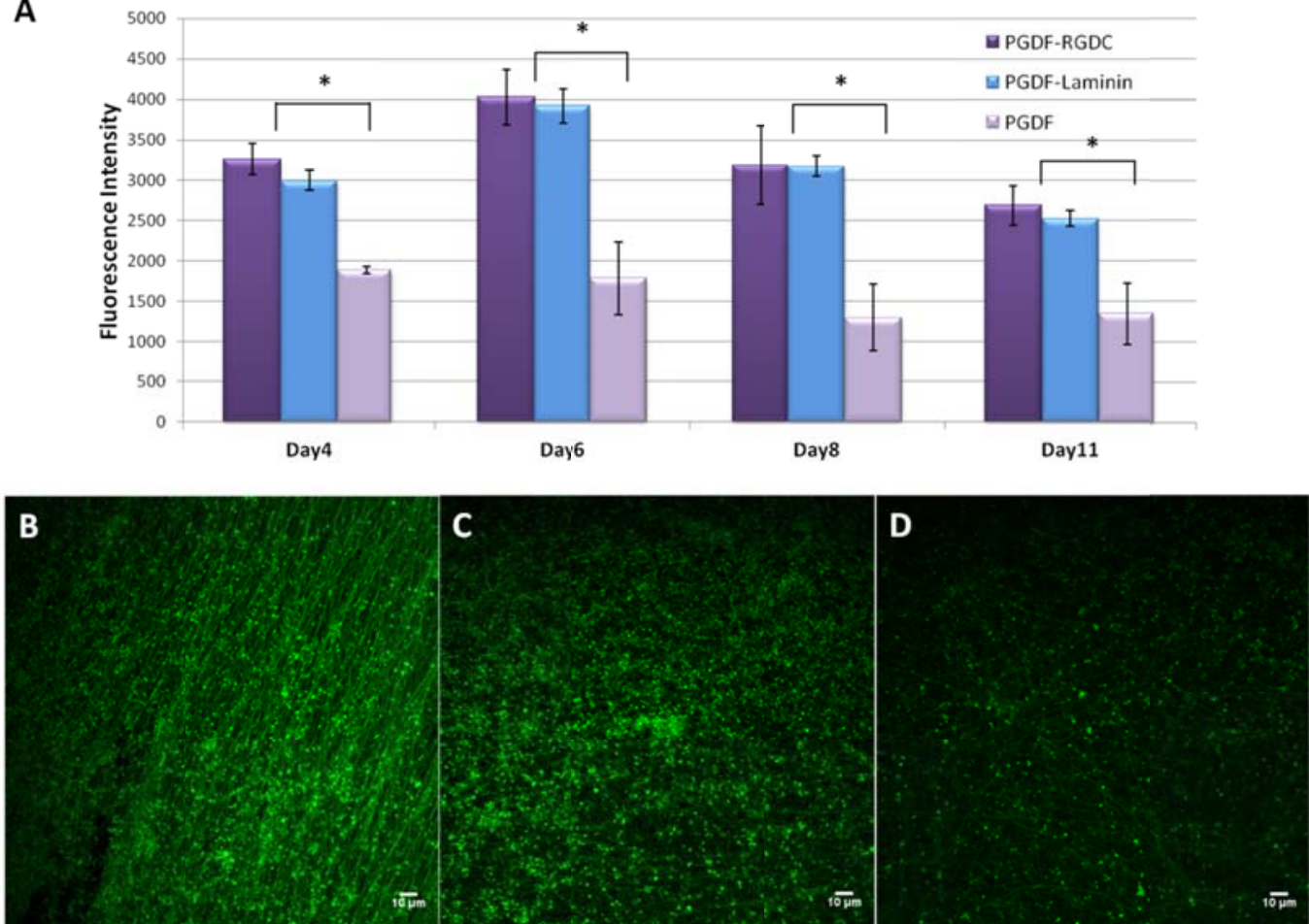

Figure 4.7. (A) Resazurin assay showing the cell viability on PGDF and peptides modified PGDF fibers on day $4,6,8$, and 11 . ${ }^{*} p<0.01$. Sample number in each group: $n=3$, experiment was repeated 3 times $(p<0.01)$. (B-D) Visualization of cells on fiber scaffolds containing (B) RGDC, $(C)$ Laminin and (D) Control after 10 days of culture. The green dots in the confocal microscope images indicate single cells which are carrying GFP. Scale bar: $10 \mu \mathrm{m}$.

\subsubsection{Cell differentiation on different fiber diameter and fiber immobilized with} peptides

To determine the effects of each of the experimental groups on cell differentiation, a variety of real time PCR marker expression levels were 
examined based upon the different diameter of the fiber (Figure 4.8A) and the presence of 2 peptide groups on day 11 (Figure $4.8 \mathrm{~B}, \mathrm{C}$ ). In addition, PGD was examined using real time PCR as an additional control to assess the effects of FA on motor neuron differentiation (Figures 4.8B, C). Although many of the markers did not express differently between the experimental groups, the $40 \%$ PGDF polymer was chosen for further study due to the clear upregulation of HB9 and downregulation of GFAP compared to the alternative concentrations of PGDF. Figures $4.8 \mathrm{~B}$ and $4.8 \mathrm{C}$ examine the $40 \%$ PGDF in detail amongst different modification of development. For ease of observation, Figure 4.8B was split into each experimental group and displayed it in Figure 4.8C. Figure 4.8C demonstrates the expression of the markers normalized to their expression in PGD. Since all of the markers in Figure $4.8 \mathrm{C}$ are expressed at a value greater than one, this indicates that the cells on the PGDF fiber significantly overexpress these markers as compared to a PGD control. It is clear that expression of Nestin, HB9 and ChAT was not significantly different between the two peptides but was significantly overexpressed as compared to PGDF. MAP2 and Olig2 were significantly more expressed in the Laminin group than in the RGDC or PGDF group. Surprisingly, ISL1 was higher expressed in the PGDF group than in either of the peptide groups. Examining the expression of GFAP there was no significant difference between any of these three groups. 

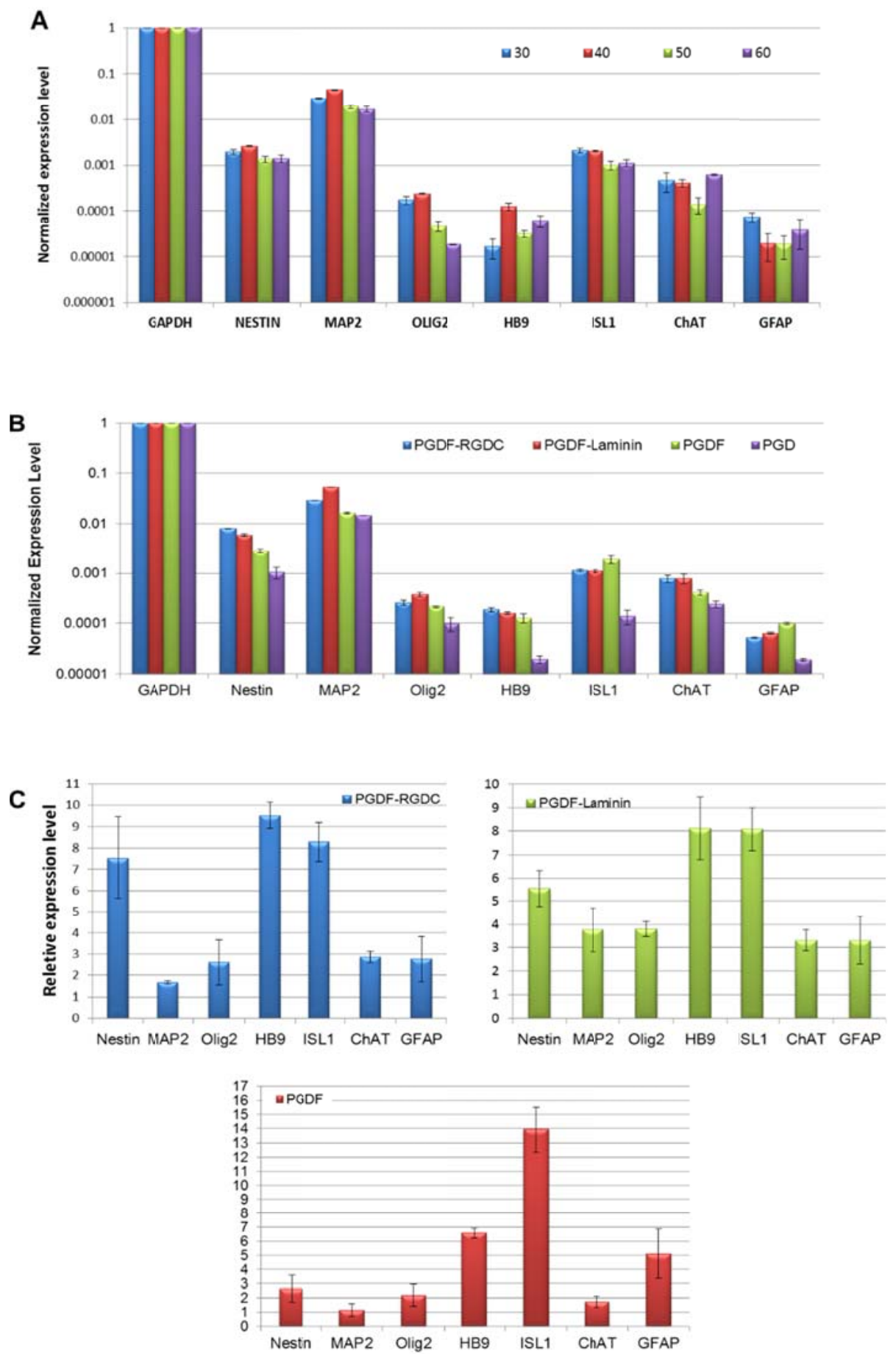

Figure 4.8. Expression of neural lineage markers through real time-PCR. Results are stratified by (A) diameter of fiber and (B, C) presence of either peptide, no peptide or no FA. In Figure 4.8A and $4.8 \mathrm{~B}$, expression levels are normalized to the expression of housekeeping gene GAPDH. In Figure $4.8 \mathrm{C}$, expression levels are normalized to the expression of the same gene in the PGD group. Average values and standard errors are from three replicate experiments. 


\subsection{Discussion}

Tissue engineering has emerged as an excellent strategy for developing protocols to maximize neural regeneration in the peripheral nervous system. Since cells cannot simply be introduced into the injury site on their own, a supportive scaffold has become a necessary part of this technique. While a scaffold may be effective for mimicking the physical, mechanical and topographical properties of the cells, the presence of bioactive materials is essential to provide continuing support to the implanted cells throughout the duration of the repair process.

PGDF was chosen for this study since PGD has been demonstrated to have sufficient biocompatibility, elasticity and the unique characteristic of shape memory[143]. The addition of FA simply made the scaffold more amenable to neural lineage survival due to its combination of neuroprotective effects and was also crucial since the double bond in its chemical structure could be simply broken to attach peptides of choice. RGDC peptides were more efficient at breaking the double bond than Laminin but only at a 50\% and 60\% PGDF concentration. The composition of the polymer was essential for devising the ideal scaffold, and increases in its concentration caused a resultant increase in viscosity, leading to a larger fiber diameter. Previous research has demonstrated an effect of fiber diameter and thus this was a factor that was chosen to be modified in the experimental design of this study [181].

PGDF can be easily dissolved in a mixture of ethanol and water. In contrast, other synthetic polymers such as PLGA and PCL require more toxic 
and expensive solvents such as hexafluoro-2-proponol and trifluoroethanol [182]. Using this more straightforward dissolution method ensured that the fabrication of fiber mats would not be a safety concern in the final implantation. This property also simplifying the monitoring of the release of FA into the surrounding structure over time via UV spectrophotometry.

In order to investigate the structural changes during polymer formation and peptide binding, a combination of Raman spectroscopy and UV spectrophotometry was used to check the presence of the internal structural bonds. DDA and FA contain carboxylic groups at both ends and glycerol has three free hydroxyl groups, all of which can be easily visualized in Raman spectra. When polymerized, the formation of a new ester bond alongside the removal of a water molecule allows the polymer to form branch structures in a three dimensional network following the curing process. After peptides binding, the decreased double bond concentration indicated that both RGDC and Laminin peptides were attached to the fibers by breaking the double bond via Michael addition.

To ensure that the polymer scaffold was effective for maintaining cell survival, cell viability was tested using a Resazurin assay. Mouse ESCs differentiated into neural cell lineages due to the presence of FA on the fiber alongside the RGDC and Laminin peptides. Both of the peptides group exhibited similar cell viability during the whole differentiation process. On the contrary, PGDF fiber scaffolds itself showed over twice difference than the other two 
groups. The confocal microscopy images also proved the importance of the peptides binding on the scaffold regarding with cell viability.

When examining the results of real time PCR the findings were intriguing. All of the following results were collected and normalized to PGD expression and each of the experimental groups demonstrated far higher expression than PGD control. These results indicated that the addition of FA alone was already a significant improvement for inducing neural lineage differentiation. Within the experimental groups, the expression of motor neuron marker HB9 and mature motor neuron marker ChAT were upregulated by the presence of the peptides over the PGDF alone. In addition, the neural stem cell marker Nestin was also upregulated in both peptide groups over the PGDF alone. Laminin was far more effective at upregulating the neuron marker MAP2 and the motor neuron precursor Olig2 than the RGDC or PGDF groups, which were not significantly different. Laminin is more frequently described as a differentiation factor than RGDC, which is more commonly believed to be an attachment factor, and thus these results are expected. The most surprising result was in the expression of the motor neuron marker ISL1, where PGDF was found to express it significantly higher than in either of the peptide groups. Previous research utilizing PDL and PSHU polymers has indicated that a Laminin coating more effectively upregulates ISL1 expression than an RGD coating, but it is unclear why the PGDF alone produces higher expression than both of these groups 
CHAPTER 5 Conclusions and Future Work

Nerve regeneration is complicated and presents great challenges to researchers. Tissue engineering provides an alternate strategy for nerve injuries. This dissertation demonstrates a highly efficient motor neuron differentiation protocol and a promising fiber scaffold for neural tissue engineering.

Chapter 2 addresses a highly efficient (65\% of cells were HB9+ and $50 \%$ of cells were ChAT+) and cost-effective method of generating mouse motor neurons using metabolites comparing to the existing protocol (See Table 5.1). By testing the efficiency of these small molecules as compared to traditional approaches, we have begun to uncover the mechanisms by which oxidative stress reduction and HDAC inhibition interplay to induce motor neuron differentiation. These mechanisms have also been demonstrated to share an underlying component - the down-regulation of glycolysis pathways - that hints at a fundamental metabolic mechanism that can be used to derive novel protocols for any stem cell differentiation approach. Using structural, molecular and functional characterization techniques, the merits of this method this verified and explored the impact of different molecular pathways, engaged through metabolite introduction, on the expression of transcriptional factors, enzymes and cytosolic markers.

Chapter 3 addresses the scaffold fabrication. PGD fiber scaffolds were successfully produced by the electrospinning process and fiber diameter was controllable through adjusting PGD concentration. The newly designed collector allows us to easily obtain fibers with adequate length and integrity. Incorporating 
riboflavin into PGD/Gelatin fibers to promote the crosslinking process under UV light was shown to improve fiber stability. The utilization of a solvent like ethanol and water for electrospinning of PGD fibrous scaffolds provides a potentially less toxic and more biocompatible fabrication method. Moreover, cell viability testing demonstrated that the addition of gelatin leads to significant improvement of cell proliferation on the scaffolds.

In Chapter 4, it is demonstrated that a novel PGDF scaffold modified with RGDC and laminin peptides is beneficial for ensuring neural cell viability and motor neuron differentiation. The natural release of FA into surrounding areas was quantified to understand the bioreactivity of the derived scaffold. In combination with UV crosslinking, the developed fiber mats will be able to withstand degradation processes long enough for neural tissue engineering applications to be viable. Cell viability and cell differentiation were demonstrated to be significantly improved with the addition of FA, RGDC and laminin. In sum, the combination of PGDF electrospun fiber scaffold and peptide immobilization demonstrates a novel approach that provides a low-cost non-toxic methodology for peripheral nervous system repair.

In future study, the new approach of using metabolites for motor neuron differentiation will be further tested with other cell types such as hESCs and iPSCs. Based on the results of mESCs and previous publications, these three metabolites should have similar effects on different pluripotent cell types with varied concentration and timing. The performance of these cells combined with fiber scaffolds will be evaluated, in vitro and in vivo. We have demonstrated two 
different peptides that contributed to neural cell survival and differentiation. It will be very interesting to test other types of peptides on this scaffold.

Table 5.1 Functional and phenotypic characteristics of MN derived from mESCs

\begin{tabular}{|c|c|c|c|c|c|}
\hline References & Cell line & $\begin{array}{l}\text { Method to } \\
\text { induce MN } \\
\text { differentiation }\end{array}$ & $\begin{array}{l}\text { MN } \\
\text { marker }\end{array}$ & $\begin{array}{l}\text { MN } \\
\text { differentiation } \\
\text { efficiency }\end{array}$ & $\begin{array}{l}\text { Functional } \\
\text { test }\end{array}$ \\
\hline $\begin{array}{l}\text { Renoncourt } \\
\text { et al., } 1998 \\
\text { [183] }\end{array}$ & $\begin{array}{l}\text { mESCs, } \\
\text { CCE line }\end{array}$ & $\begin{array}{l}\text { Embryo Body } \\
\text { (EB) treated } \\
\text { with RA }\end{array}$ & $\begin{array}{l}\text { ISL1, } \\
\text { HB9, } \\
\text { ChAT } \\
\text { and } \\
\text { VAChT }\end{array}$ & $\begin{array}{l}40-60 \% \text { of } \\
\text { Islet1+ } \\
\text { neurons;12\% } \\
\text { of Islet1+ } \\
\text { were HB9+ }\end{array}$ & $\mathrm{N}$ \\
\hline $\begin{array}{l}\text { Wichterle et } \\
\text { al., 2002 } \\
{[184]}\end{array}$ & $\begin{array}{l}\text { mESCs, } \\
\text { HBG3 line }\end{array}$ & $\begin{array}{l}\text { EB treated } \\
\text { with RA and } \\
\text { SHH }\end{array}$ & $\begin{array}{l}\text { Hoxc6, } \\
\text { Hoxc7, } \\
\text { Hoxc8, } \\
\text { Nkx6.1, } \\
\text { Olig2, } \\
\text { Lhx3, } \\
\text { Lim1, } \\
\text { ISL1, } \\
\text { VAChT, } \\
\text { ChAT }\end{array}$ & $\begin{array}{l}20-30 \% \text { of } \\
\text { neurons were } \\
\text { HB9+ }\end{array}$ & $\mathrm{Y}$ \\
\hline $\begin{array}{l}\text { Deshpande } \\
\text { et al., } 2006 \\
\text { [185] }\end{array}$ & $\begin{array}{l}\text { mESCs, } \\
\text { HBG3 line }\end{array}$ & $\begin{array}{l}\text { EB treated } \\
\text { with RA and } \\
\text { SHH }\end{array}$ & $\begin{array}{l}\text { Lhx3, } \\
\text { HB9 }\end{array}$ & $\begin{array}{l}35-40 \% \text { of } \\
\text { neurons were } \\
\text { ChAT+ }\end{array}$ & $Y$ \\
\hline $\begin{array}{l}\text { Hamada et } \\
\text { al., 2006 } \\
{[186]}\end{array}$ & $\begin{array}{l}\text { mESCs, } \\
\text { E14.1 line } \\
\text { transfected } \\
\text { with } \\
\text { MASH1 }\end{array}$ & $\begin{array}{l}\text { EB treated } \\
\text { with } R A\end{array}$ & $\begin{array}{l}\text { ISL1, } \\
\operatorname{Lim} 1 / 2, \\
\text { HB9 }\end{array}$ & $\begin{array}{l}50 \% \text { of } \\
\text { MASH1 } \\
\text { transfected } \\
\text { cells were } \\
\text { Islet1+ } \\
\text { neurons }\end{array}$ & $Y$ \\
\hline $\begin{array}{l}\text { López- } \\
\text { González et } \\
\text { al., } 2009 \\
{[187]} \\
\end{array}$ & $\begin{array}{l}\text { mESCs, } \\
\text { HBG3 line }\end{array}$ & $\begin{array}{l}\text { EB treated } \\
\text { with RA and } \\
\text { SHH }\end{array}$ & $\begin{array}{l}\text { ISL1, } \\
\text { ChAT }\end{array}$ & $\begin{array}{l}15-20 \% \text { of } \\
\text { neurons were } \\
\text { GFP+MN }\end{array}$ & $\mathrm{Y}$ \\
\hline $\begin{array}{l}\text { Wu et al., } \\
2011 \text { [188] }\end{array}$ & $\begin{array}{l}\text { mESCs } \\
\text { from a } \\
\text { SMA } \\
\text { animal } \\
\text { model }\end{array}$ & $\begin{array}{l}\text { EB treated } \\
\text { with Noggin, } \\
\mathrm{RA} \text { and } \mathrm{SHH}\end{array}$ & $\begin{array}{l}\text { ISL1, } \\
\text { HB9 and } \\
\text { ChAT }\end{array}$ & $40-50 \% \mathrm{MN}$ & $N$ \\
\hline
\end{tabular}




\section{LIST OF REFERENCES}

1. Schmidt, C.E. and J.B. Leach, Neural tissue engineering: strategies for repair and regeneration. Annual review of biomedical engineering, 2003. 5(1): p. 293-347.

2. Lucas, S.M., N.J. Rothwell, and R.M. Gibson, The role of inflammation in CNS injury and disease. British journal of pharmacology, 2006. 147(S1): $p$. S232-S240.

3. Sims, N.R. and H. Muyderman, Mitochondria, oxidative metabolism and cell death in stroke. Biochimica et Biophysica Acta (BBA)-Molecular Basis of Disease, 2010. 1802(1): p. 80-91.

4. Fisher, M., et al., Update of the stroke therapy academic industry roundtable preclinical recommendations. Stroke, 2009. 40(6): p. 22442250.

5. Organization, W.H., The World Health Report 2004-Changing History, Annex Table 2: Deaths By Cause, Sex and Mortality Stratum in WHO Regions, Estimates For 2002. World Health Organization, Geneva, Switzerland, 2004.

6. Forraz, N., et al., Experimental therapies for repair of the central nervous system: stem cells and tissue engineering. Journal of tissue engineering and regenerative medicine, 2013. 7(7): p. 523-536.

7. Burnett, M.G. and E.L. Zager, Pathophysiology of peripheral nerve injury: a brief review. Neurosurgical focus, 2004. 16(5): p. 1-7.

8. Johnson, E.O. and P.N. Soucacos, Nerve repair: experimental and clinical evaluation of biodegradable artificial nerve guides. Injury, 2008. 39(3): p. 30-36.

9. Chen, M.B., F. Zhang, and W.C. Lineaweaver, Luminal fillers in nerve conduits for peripheral nerve repair. Annals of plastic surgery, 2006. 57(4): p. $462-471$.

10. Griffith, L.G. and G. Naughton, Tissue engineering--current challenges and expanding opportunities. Science, 2002. 295(5557): p. 1009-1014.

11. Dahlin, R.L., F.K. Kasper, and A.G. Mikos, Polymeric nanofibers in tissue engineering. Tissue Engineering Part B: Reviews, 2011. 17(5): p. 349-364.

12. Pham, Q.P., U. Sharma, and A.G. Mikos, Electrospinning of polymeric nanofibers for tissue engineering applications: a review. Tissue engineering, 2006. 12(5): p. 1197-1211. 
13. Xie, J., X. Li, and Y. Xia, Putting electrospun nanofibers to work for biomedical research. Macromolecular rapid communications, 2008. 29(22): p. $1775-1792$.

14. Yang, F., et al., Electrospinning of nano/micro scale poly (L-lactic acid) aligned fibers and their potential in neural tissue engineering. Biomaterials, 2005. 26(15): p. 2603-2610.

15. Sahoo, S., et al., Growth factor delivery through electrospun nanofibers in scaffolds for tissue engineering applications. Journal of Biomedical Materials Research Part A, 2010. 93(4): p. 1539-1550.

16. Sahoo, S., et al., Characterization of a novel polymeric scaffold for potential application in tendon/ligament tissue engineering. Tissue engineering, 2006. 12(1): p. 91-99.

17. Anastas, P.T. and J.C. Warner, Green Chemistry: Theory and Practice. 1998: Oxford University Press. p30.

18. Wang, Y., et al., A tough biodegradable elastomer. Nature biotechnology, 2002. 20(6): p. 602-606.

19. Migneco, F., et al., Poly(glycerol-dodecanoate), a biodegradable polyester for medical devices and tissue engineering scaffolds. Biomaterials, 2009. 30(33): p. 6479-84.

20. Migneco, F., et al., Poly (glycerol-dodecanoate), a biodegradable polyester for medical devices and tissue engineering scaffolds. Biomaterials, 2009. 30(33): p. 6479-6484.

21. Panunzi, S., A. De Gaetano, and G. Mingrone, Approximate linear confidence and curvature of a kinetic model of dodecanedioic acid in humans. Am J Physiol Endocrinol Metab, 2005. 289(5): p. E915-22.

22. Mingrone, G., et al., Pharmacokinetic profile of dodecanedioic acid, a proposed alternative fuel substrate. JPEN J Parenter Enteral Nutr, 1994. 18(3): p. 225-30.

23. Van de Witte, P., et al., Phase separation processes in polymer solutions in relation to membrane formation. Journal of Membrane Science, 1996. 117(1): p. 1-31.

24. Hartgerink, J.D., E. Beniash, and S.I. Stupp, Self-assembly and mineralization of peptide-amphiphile nanofibers. Science, 2001. 294(5547): p. 1684-1688. 
25. Matthews, J.A., et al., Electrospinning of collagen nanofibers. Biomacromolecules, 2002. 3(2): p. 232-238.

26. Gupta, P., et al., Electrospinning of linear homopolymers of poly (methyl methacrylate): exploring relationships between fiber formation, viscosity, molecular weight and concentration in a good solvent. Polymer, 2005. 46(13): p. 4799-4810.

27. McKee, M.G., et al., Phospholipid nonwoven electrospun membranes. Science, 2006. 311(5759): p. 353-355.

28. Fong, H., I. Chun, and D. Reneker, Beaded nanofibers formed during electrospinning. Polymer, 1999. 40(16): p. 4585-4592.

29. Ki, C.S., et al., Characterization of gelatin nanofiber prepared from gelatin-formic acid solution. Polymer, 2005. 46(14): p. 5094-5102.

30. Huang, L., et al., Engineered collagen-PEO nanofibers and fabrics. Journal of Biomaterials Science, Polymer Edition, 2001. 12(9): p. 979-993.

31. Lee, J.S., et al., Role of molecular weight of atactic poly (vinyl alcohol)(PVA) in the structure and properties of PVA nanofabric prepared by electrospinning. Journal of Applied Polymer Science, 2004. 93(4): $p$. 1638-1646.

32. Hou, H., et al., Poly (p-xylylene) nanotubes by coating and removal of ultrathin polymer template fibers. Macromolecules, 2002. 35(7): p. 24292431.

33. Deitzel, J., et al., The effect of processing variables on the morphology of electrospun nanofibers and textiles. Polymer, 2001. 42(1): p. 261-272.

34. Chronakis, I.S., S. Grapenson, and A. Jakob, Conductive polypyrrole nanofibers via electrospinning: electrical and morphological properties. Polymer, 2006. 47(5): p. 1597-1603.

35. Zong, X., et al., Structure and process relationship of electrospun bioabsorbable nanofiber membranes. Polymer, 2002. 43(16): p. 44034412.

36. Mit-uppatham, C., M. Nithitanakul, and P. Supaphol, Ultrafine Electrospun Polyamide-6 Fibers: Effect of Solution Conditions on Morphology and Average Fiber Diameter. Macromolecular Chemistry and Physics, 2004. 205(17): p. 2327-2338.

37. Zhang, C., et al., Study on morphology of electrospun poly (vinyl alcohol) mats. European polymer journal, 2005. 41(3): p. 423-432. 
38. Kim, B., et al., Poly (acrylic acid) nanofibers by electrospinning. Materials letters, 2005. 59(7): p. 829-832.

39. Hohman, M.M., et al., Electrospinning and electrically forced jets. I. Stability theory. Physics of Fluids, 2001. 13: p. 2201.

40. Haghi, A. and M. Akbari, Trends in electrospinning of natural nanofibers. physica status solidi (a), 2007. 204(6): p. 1830-1834.

41. Pham, Q.P., U. Sharma, and A.G. Mikos, Electrospun poly (ع-caprolactone) microfiber and multilayer nanofiber/microfiber scaffolds: characterization of scaffolds and measurement of cellular infiltration. Biomacromolecules, 2006. 7(10): p. 2796-2805.

42. Demir, M.M., et al., Electrospinning of polyurethane fibers. Polymer, 2002. 43(11): p. 3303-3309.

43. Buchko, C.J., et al., Processing and microstructural characterization of porous biocompatible protein polymer thin films. Polymer, 1999. 40(26): $p$. 7397-7407.

44. Katti, D.S., et al., Bioresorbable nanofiber-based systems for wound healing and drug delivery: Optimization of fabrication parameters. Journal of Biomedical Materials Research Part B: Applied Biomaterials, 2004. 70(2): p. 286-296.

45. Pavlov, M.P., et al., Fibers and 3D Mesh Scaffolds from Biodegradable Starch-Based Blends: Production and Characterization. Macromolecular bioscience, 2004. 4(8): p. 776-784.

46. Reneker, D.H. and I. Chun, Nanometre diameter fibres of polymer, produced by electrospinning. Nanotechnology, 1999. 7(3): p. 216.

47. Wannatong, L., A. Sirivat, and P. Supaphol, Effects of solvents on electrospun polymeric fibers: preliminary study on polystyrene. Polymer International, 2004. 53(11): p. 1851-1859.

48. Yuan, X., et al., Morphology of ultrafine polysulfone fibers prepared by electrospinning. Polymer International, 2004. 53(11): p. 1704-1710.

49. Kim, K.H., et al., Biological efficacy of silk fibroin nanofiber membranes for guided bone regeneration. Journal of biotechnology, 2005. 120(3): p. 327339.

50. Megelski, S., et al., Micro-and nanostructured surface morphology on electrospun polymer fibers. Macromolecules, 2002. 35(22): p. 8456-8466. 
51. Wang, X., et al., Formation of water-resistant hyaluronic acid nanofibers by blowing-assisted electro-spinning and non-toxic post treatments. Polymer, 2005. 46(13): p. 4853-4867.

52. Sanders, E.H., et al., Two-phase electrospinning from a single electrified jet: Microencapsulation of aqueous reservoirs in poly (ethylene-co-vinyl acetate) fibers. Macromolecules, 2003. 36(11): p. 3803-3805.

53. Li, D. and Y. Xia, Electrospinning of nanofibers: reinventing the wheel? Advanced materials, 2004. 16(14): p. 1151-1170.

54. Kumbar, S.G., et al., Recent patents on electrospun biomedical nanostructures: an overview. Recent Patents on Biomedical Engineering, 2008. 1(1): p. 68-78.

55. Deitzel, J., et al., The effect of processing variables on the morphology of electrospun nanofibers and textiles. Polymer, 2001. 42(1): p. 261-272.

56. Jalili, R., M. Morshed, and S.A.H. Ravandi, Fundamental parameters affecting electrospinning of PAN nanofibers as uniaxially aligned fibers. Journal of Applied Polymer Science, 2006. 101(6): p. 4350-4357.

57. Casper, C.L., et al., Controlling surface morphology of electrospun polystyrene fibers: Effect of humidity and molecular weight in the electrospinning process. Macromolecules, 2004. 37(2): p. 573-578.

58. Li, D., Y. Wang, and Y. Xia, Electrospinning nanofibers as uniaxially aligned arrays and layer-by-layer stacked films. Advanced materials, 2004. 16(4): p. 361-366.

59. Baumgarten, P.K., Electrostatic spinning of acrylic microfibers. Journal of colloid and interface science, 1971. 36(1): p. 71-79.

60. Barnes, C.P., et al., Nanofiber technology: designing the next generation of tissue engineering scaffolds. Advanced drug delivery reviews, 2007. 59(14): p. 1413-1433.

61. Li, W.J., R.L. Mauck, and R.S. Tuan, Electrospun nanofibrous scaffolds: production, characterization, and applications for tissue engineering and drug delivery. Journal of Biomedical Nanotechnology, 2005. 1(3): p. 259275.

62. Huang, Z.M., et al., Electrospinning and mechanical characterization of gelatin nanofibers. Polymer, 2004. 45(15): p. 5361-5368. 
63. Christopherson, G.T., H. Song, and H.Q. Mao, The influence of fiber diameter of electrospun substrates on neural stem cell differentiation and proliferation. Biomaterials, 2009. 30(4): p. 556-564.

64. Yoshimoto, $\mathrm{H}$., et al., $A$ biodegradable nanofiber scaffold by electrospinning and its potential for bone tissue engineering. Biomaterials, 2003. 24(12): p. 2077-2082.

65. Li, W.J., et al., Biological response of chondrocytes cultured in three-dimensional nanofibrous poly ( $\epsilon$-caprolactone) scaffolds. Journal of Biomedical Materials Research Part A, 2003. 67(4): p. 1105-1114.

66. Kumbar, S.G., et al., Electrospun poly (lactic acid-<i>co</i>-glycolic acid) scaffolds for skin tissue engineering. Biomaterials, 2008. 29(30): p. 41004107.

67. Brons, I.G., et al., Derivation of pluripotent epiblast stem cells from mammalian embryos. Nature, 2007. 448(7150): p. 191-5.

68. Orlova, Y., et al., Electrospun nanofibers as a tool for architecture control in engineered cardiac tissue. Biomaterials, 2011. 32(24): p. 5615-5624.

69. Gokhale, P.J. and P.W. Andrews, The development of pluripotent stem cells. Current Opinion in Genetics \&amp; Development, 2012. 22(5): p. 403-408.

70. Stevens, L.C. and C.C. Little, Spontaneous Testicular Teratomas in an Inbred Strain of Mice. Proc Natl Acad Sci U S A, 1954. 40(11): p. 1080-7.

71. Kleinsmith, L.J. and G.B. Pierce, Jr., Multipotentiality of Single Embryonal Carcinoma Cells. Cancer Res, 1964. 24: p. 1544-51.

72. Evans, M.J. and M.H. Kaufman, Establishment in culture of pluripotential cells from mouse embryos. Nature, 1981. 292(5819): p. 154-6.

73. Martin, G.R., Isolation of a pluripotent cell line from early mouse embryos cultured in medium conditioned by teratocarcinoma stem cells. Proc Natl Acad Sci U S A, 1981. 78(12): p. 7634-8.

74. Williams, R.L., et al., Myeloid leukaemia inhibitory factor maintains the developmental potential of embryonic stem cells. Nature, 1988. 336(6200): p. 684-7.

75. Holden, S., et al., Human and mouse embryonal carcinoma cells in culture share an embryonic antigen (F9). Nature, 1977. 270(5637): p. 518-20. 
76. Thomson, J.A., et al., Embryonic stem cell lines derived from human blastocysts. Science, 1998. 282(5391): p. 1145-7.

77. Boeuf, H., et al., Leukemia inhibitory factor-dependent transcriptional activation in embryonic stem cells. J Cell Biol, 1997. 138(6): p. 1207-17.

78. $\mathrm{Xu}, \mathrm{C}$., et al., Feeder-free growth of undifferentiated human embryonic stem cells. Nat Biotechnol, 2001. 19(10): p. 971-4.

79. Levenstein, M.E., et al., Basic fibroblast growth factor support of human embryonic stem cell self-renewal. Stem Cells, 2006. 24(3): p. 568-74.

80. Vallier, L., M. Alexander, and R.A. Pedersen, Activin/Nodal and FGF pathways cooperate to maintain pluripotency of human embryonic stem cells. J Cell Sci, 2005. 118(Pt 19): p. 4495-509.

81. James, D., et al., TGFbeta/activin/nodal signaling is necessary for the maintenance of pluripotency in human embryonic stem cells. Development, 2005. 132(6): p. 1273-82.

82. Tesar, P.J., et al., New cell lines from mouse epiblast share defining features with human embryonic stem cells. Nature, 2007. 448(7150): p. 196-9.

83. Drukker, M. and N. Benvenisty, The immunogenicity of human embryonic stem-derived cells. Trends Biotechnol, 2004. 22(3): p. 136-41.

84. Gurdon, J.B., The developmental capacity of nuclei taken from intestinal epithelium cells of feeding tadpoles. J Embryol Exp Morphol, 1962. 10: p. 622-40.

85. Wilmut, I., et al., Viable offspring derived from fetal and adult mammalian cells. Nature, 1997. 385(6619): p. 810-3.

86. Rideout, W.M., 3rd, et al., Correction of a genetic defect by nuclear transplantation and combined cell and gene therapy. Cell, 2002. 109(1): $p$. 17-27.

87. Byrne, J.A., et al., Producing primate embryonic stem cells by somatic cell nuclear transfer. Nature, 2007. 450(7169): p. 497-502.

88. Hochedlinger, K. and R. Jaenisch, Nuclear reprogramming and pluripotency. Nature, 2006. 441(7097): p. 1061-7.

89. Takahashi, K. and S. Yamanaka, Induction of pluripotent stem cells from mouse embryonic and adult fibroblast cultures by defined factors. Cell, 2006. 126(4): p. 663-76. 
90. Wernig, M., et al., In vitro reprogramming of fibroblasts into a pluripotent ES-cell-like state. Nature, 2007. 448(7151): p. 318-324.

91. Takahashi, K., et al., Induction of pluripotent stem cells from adult human fibroblasts by defined factors. Cell, 2007. 131(5): p. 861-72.

92. $\mathrm{Yu}, \mathrm{J}$., et al., Induced pluripotent stem cell lines derived from human somatic cells. Science, 2007. 318(5858): p. 1917-20.

93. Park, I.H., et al., Reprogramming of human somatic cells to pluripotency with defined factors. Nature, 2008. 451(7175): p. 141-6.

94. Lowry, W.E., et al., Generation of human induced pluripotent stem cells from dermal fibroblasts. Proc Natl Acad Sci U S A, 2008. 105(8): p. 2883-8.

95. Murry, C.E. and G. Keller, Differentiation of embryonic stem cells to clinically relevant populations: lessons from embryonic development. Cell, 2008. 132(4): p. 661-80.

96. Kawasaki, H., Induction of midbrain dopaminergic neurons from ES cells by stromal cell-derived inducing activity. Neuron, 2000. 28: p. 31-40.

97. Lee, H., Directed differentiation and transplantation of human embryonic stem cell-derived motoneurons. Stem Cells, 2007. 25: p. 1931-1939.

98. Munoz-Sanjuan, I. and A.H. Brivanlou, Neural induction, the default model and embryonic stem cells. Nat. Rev. Neurosci., 2002. 3: p. 271-280.

99. Joannides, A.J., et al., A Scaleable and Defined System for Generating Neural Stem Cells from Human Embryonic Stem Cells. Stem Cells, 2007. 25(3): p. 731-737.

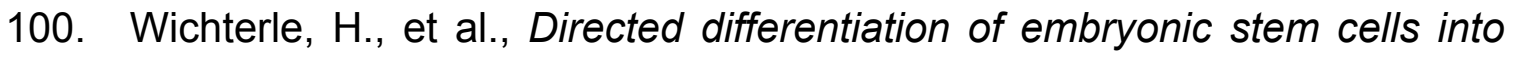
motor neurons. Cell, 2002. 110(3): p. 385-97.

101. Lee, S.H., et al., Efficient generation of midbrain and hindbrain neurons from mouse embryonic stem cells. Nat Biotechnol, 2000. 18(6): p. 675-9.

102. Perrier, A.L., et al., Derivation of midbrain dopamine neurons from human embryonic stem cells. Proc Natl Acad Sci U S A, 2004. 101(34): p. 125438.

103. Yan, Y., et al., Directed differentiation of dopaminergic neuronal subtypes from human embryonic stem cells. Stem Cells, 2005. 23(6): p. 781-90. 
104. Nistor, G.I., et al., Human embryonic stem cells differentiate into oligodendrocytes in high purity and myelinate after spinal cord transplantation. Glia, 2005. 49(3): p. 385-96.

105. Finsterer, J. and J.-M. Burgunder, Recent progress in the genetics of motor neuron disease. European journal of medical genetics, 2014. 57(2): p. 103-112.

106. Liu, Y. and S.C. Zhang, Human stem cells as a model of motoneuron development and diseases. Annals of the New York Academy of Sciences, 2010. 1198(1): p. 192-200.

107. Hu, B.-Y. and S.-C. Zhang, Differentiation of spinal motor neurons from pluripotent human stem cells. Nature protocols, 2009. 4(9): p. 1295-1304.

108. Hu, B.-Y. and S.-C. Zhang, Directed differentiation of neural-stem cells and subtype-specific neurons from hESCs, in Cellular Programming and Reprogramming. 2010, Springer. p. 123-137.

109. Li, X.-J., et al., Specification of motoneurons from human embryonic stem cells. Nature biotechnology, 2005. 23(2): p. 215-221.

110. Wu, X., et al., Purmorphamine induces osteogenesis by activation of the hedgehog signaling pathway. Chemistry \& biology, 2004. 11(9): p. 12291238.

111. Schulz, T.C., et al., Directed neuronal differentiation of human embryonic stem cells. BMC neuroscience, 2003. 4(1): p. 27.

112. Lee, H., et al., Directed differentiation and transplantation of human embryonic stem cell-derived motoneurons. Stem cells, 2007. 25(8): p. 1931-1939.

113. Chambers, S.M., et al., Highly efficient neural conversion of human ES and iPS cells by dual inhibition of SMAD signaling. Nature biotechnology, 2009. 27(3): p. 275-280.

114. Panseri, S., et al., Electrospun micro-and nanofiber tubes for functional nervous regeneration in sciatic nerve transections. BMC biotechnology, 2008. 8(1): p. 39.

115. Xie, J., et al., The differentiation of embryonic stem cells seeded on electrospun nanofibers into neural lineages. Biomaterials, 2009. 30(3): p. 354-362. 
116. Ghasemi-Mobarakeh, L., et al., Electrical stimulation of nerve cells using conductive nanofibrous scaffolds for nerve tissue engineering. Tissue Engineering Part A, 2009. 15(11): p. 3605-3619.

117. Cullen, D.K., et al., Developing a tissue-engineered neural-electrical relay using encapsulated neuronal constructs on conducting polymer fibers. Journal of Neural Engineering, 2008. 5(4): p. 374.

118. Liu, X., et al., Conducting polymers with immobilised fibrillar collagen for enhanced neural interfacing. Biomaterials, 2011. 32(30): p. 7309-7317.

119. Liu, T., et al., Sustained release of neurotrophin-3 and chondroitinase ABC from electrospun collagen nanofiber scaffold for spinal cord injury repair. Journal of Biomedical Materials Research Part A, 2012.

120. Lam, H.J., et al., In vitro regulation of neural differentiation and axon growth by growth factors and bioactive nanofibers. Tissue Engineering Part A, 2010. 16(8): p. 2641-2648.

121. Lee, J.Y., et al., Nerve growth factor-immobilized electrically conducting fibrous scaffolds for potential use in neural engineering applications. NanoBioscience, IEEE Transactions on, 2012. 11(1): p. 15-21.

122. Reinhardt, P., et al., Derivation and expansion using only small molecules of human neural progenitors for neurodegenerative disease modeling. PloS one, 2013. 8(3): p. e59252.

123. Pera, E.M., et al., Integration of IGF, FGF, and anti-BMP signals via Smad1 phosphorylation in neural induction. Genes \& development, 2003. 17(24): p. 3023-3028.

124. Byfield, S.D., et al., SB-505124 is a selective inhibitor of transforming growth factor- $\beta$ type I receptors ALK4, ALK5, and ALK7. Molecular Pharmacology, 2004. 65(3): p. 744-752.

125. Heeg-Truesdell, E. and C. LaBonne, Neural induction in $<$ i> Xenopus $</ i>$ requires inhibition of Wnt- $\beta$-catenin signaling. Developmental biology, 2006. 298(1): p. 71-86.

126. Wilson, L. and M. Maden, The mechanisms of dorsoventral patterning in the vertebrate neural tube. Developmental biology, 2005. 282(1): p. 1-13.

127. Ashrafian, $\mathrm{H}$., et al., Fumarate is cardioprotective via activation of the Nrf2 antioxidant pathway. Cell metabolism, 2012. 15(3): p. 361-371.

128. Albrecht, P., et al., Effects of dimethyl fumarate on neuroprotection and immunomodulation. J Neuroinflammation, 2012. 9(163): p. 2094-9. 
129. Moharregh-Khiabani, D., et al., Fumaric acid and its esters: an emerging treatment for multiple sclerosis. Current neuropharmacology, 2009. 7(1): p. 60.

130. Chen, J., H. Lin, and M. Hu, Absorption and metabolism of genistein and its five isoflavone analogs in the human intestinal Caco-2 model. Cancer chemotherapy and pharmacology, 2005. 55(2): p. 159-169.

131. Matsumoto, M., [Inhibitors for protein tyrosine kinases, erbstatin, genistein and herbimycin A, induce differentiation of human neural tumor cell lines]. Nihon geka hokan. Archiv fur japanische Chirurgie, 1991. 60(2): p. 113121.

132. Pan, M., et al., Effects of genistein and daidzein on hippocampus neuronal cell proliferation and BDNF expression in H19-7 neural cell line. The journal of nutrition, health \& aging, 2012. 16(4): p. 389-394.

133. Huang, Y.-H. and Q.-H. Zhang, Genistein reduced the neural apoptosis in the brain of ovariectomised rats by modulating mitochondrial oxidative stress. British journal of nutrition, 2010. 104(09): p. 1297-1303.

134. Shakèd, M., et al., Histone deacetylases control neurogenesis in embryonic brain by inhibition of BMP2/4 signaling. PloS one, 2008. 3(7): p. e2668.

135. Yao, X., et al., Histone deacetylase inhibitor promotes differentiation of embryonic stem cells into neural cells in adherent monoculture. Chinese Medical Journal (English Edition), 2010. 123(6): p. 734.

136. Lyssiotis, C.A., et al., Inhibition of histone deacetylase activity induces developmental plasticity in oligodendrocyte precursor cells. Proceedings of the National Academy of Sciences, 2007. 104(38): p. 14982-14987.

137. Ross, A.C. and R. Zolfaghari, Cytochrome P450s in the regulation of cellular retinoic acid metabolism. Annual review of nutrition, 2011. 31: p. 65.

138. Leschelle, X., et al., Butyrate metabolism upstream and downstream acetyl-CoA synthesis and growth control of human colon carcinoma cells. European Journal of Biochemistry, 2000. 267(21): p. 6435-6442.

139. Ochocki, J.D. and M.C. Simon, Nutrient-sensing pathways and metabolic regulation in stem cells. The Journal of cell biology, 2013. 203(1): p. 23-33.

140. Varum, S., et al., Energy metabolism in human pluripotent stem cells and their differentiated counterparts. PloS one, 2011. 6(6): p. e20914. 
141. O'Brien, J., et al., Investigation of the Alamar Blue (resazurin) fluorescent dye for the assessment of mammalian cell cytotoxicity. Eur J Biochem, 2000. 267(17): p. 5421-6.

142. Ansar Ahmed, S., R.M. Gogal Jr, and J.E. Walsh, A new rapid and simple non-radioactive assay to monitor and determine the proliferation of lymphocytes: an alternative to $[<\sup >3</$ sup $>\mathrm{H}]$ thymidine incorporation assay. Journal of immunological methods, 1994. 170(2): p. 211-224.

143. Dai, X., K. Kathiria, and Y.-C. Huang, Electrospun fiber scaffolds of poly (glycerol-dodecanedioate) and its gelatin blended polymers for soft tissue engineering. Biofabrication, 2014. 6(3): p. 035005.

144. O'Brien, J., et al., Investigation of the Alamar Blue (resazurin) fluorescent dye for the assessment of mammalian cell cytotoxicity. European Journal of Biochemistry, 2000. 267(17): p. 5421-5426.

145. Land, B.R., R.A. Wyttenbach, and B.R. Johnson, Tools for physiology labs: an inexpensive high-performance amplifier and electrode for extracellular recording. Journal of neuroscience methods, 2001. 106(1): p. 47-55.

146. Boinagrov, D., et al., Upper Threshold of Extracellular Stimulation in Retinal Ganglion Cells. Investigative Ophtalmology and Visual Science, 2011. 52(6): p. 4956.

147. Mitsuishi, Y., et al., Nrf2 redirects glucose and glutamine into anabolic pathways in metabolic reprogramming. Cancer cell, 2012. 22(1): p. 66-79.

148. Tandon, V., B. Das, and N. Saha, Anthelmintic efficacy of $<$ i> Flemingia vestita</i>(Fabaceae): effect of genistein on glycogen metabolism in the cestode, $<$ i> Raillietina echinobothrida</i>. Parasitology international, 2003. 52(2): p. 179-183.

149. Blouin, J.M., et al., Butyrate elicits a metabolic switch in human colon cancer cells by targeting the pyruvate dehydrogenase complex. International Journal of Cancer, 2011. 128(11): p. 2591-2601.

150. Xun, Z., et al., Retinoic acid induces a metabolic switch in SH-SY5Y cells from glycolysis to oxidative phosphorylation. The FASEB Journal, 2012. 26: p. 967.1 .

151. Bell, E., Strategy for the selection of scaffolds for tissue engineering. Tissue Eng, 1995. 1(2): p. 163-79.

152. Rozario, T. and D.W. DeSimone, The extracellular matrix in development and morphogenesis: a dynamic view. Dev Biol, 2010. 341(1): p. 126-40. 
153. Reneker, D.H. and I. Chun, Nanometre diameter fibres of polymer, produced by electrospinning. Nanotechnology, 1996. 7(3): p. 216.

154. Bellan, L.M. and H.G. Craighead, Applications of controlled electrospinning systems. Polymers for Advanced Technologies, 2011. 22(3): p. 304-309.

155. Zhou, C.H., et al., Chemoselective catalytic conversion of glycerol as a biorenewable source to valuable commodity chemicals. Chem Soc Rev, 2008. 37(3): p. 527-49.

156. Folahan O. Ayorinde, et al., Synthesis of dodecanedioic acid from vernonia galamensis oil. Journal of Oil \& Fat Industries, 1989. 66(5): p. 690-692.

157. Grego, A.V. and G. Mingrone, Dicarboxylic acids, an alternate fuel substrate in parenteral nutrition: an update. Clin Nutr, 1995. 14(3): p. 1438.

158. Mingrone, G., et al., Plasma clearance and oxidation of dodecanedioic acid in humans. JPEN J Parenter Enteral Nutr, 1996. 20(1): p. 38-42.

159. Weir, N., et al., Processing, annealing and sterilisation of poly-L-lactide. Biomaterials, 2004. 25(18): p. 3939-3949.

160. Rydevik, B.L., et al., An in vitro mechanical and histological study of acute stretching on rabbit tibial nerve. Journal of Orthopaedic Research, 1990. 8(5): p. 694-701.

161. Hung, T.-K., et al., Stress-strain relationship of the spinal cord of anesthetized cats. Journal of biomechanics, 1981. 14(4): p. 269-276.

162. Agache, P., et al., Mechanical properties and Young's modulus of human skin in vivo. Archives of dermatological research, 1980. 269(3): p. 221-232.

163. Kang, H.-W., Y. Tabata, and Y. Ikada, Fabrication of porous gelatin scaffolds for tissue engineering. Biomaterials, 1999. 20(14): p. 1339-1344.

164. Rujitanaroj, P.-o., N. Pimpha, and P. Supaphol, Wound-dressing materials with antibacterial activity from electrospun gelatin fiber mats containing silver nanoparticles. Polymer, 2008. 49(21): p. 4723-4732.

165. Laba, D. and J. Gambino, Flexible gelatin free encapsulation material useful for pharmaceuticals, paint balls and other formulations. 2001, Google Patents. 
166. Reneker, D.H. and A.L. Yarin, Electrospinning jets and polymer nanofibers. Polymer, 2008. 49(10): p. 2387-2425.

167. Miller, J.V. and E.G. Bartick, Forensic analysis of single fibers by Raman spectroscopy. Applied spectroscopy, 2002. 56(1): p. 1729-1732.

168. Ansar Ahmed, S., R.M. Gogal, and J.E. Walsh, A new rapid and simple non-radioactive assay to monitor and determine the proliferation of lymphocytes: an alternative to $[<\sup >3</$ sup $>\mathrm{H}$ ] thymidine incorporation assay. Journal of immunological methods, 1994. 170(2): p. 211-224.

169. Sheu, W.-S., Molecular Weight Averages and Polydispersity of Polymers. Journal of Chemical Education, 2001. 78(4): p. 554.

170. Mendelovici, E., R.L. Frost, and T. Kloprogge, Cryogenic Raman spectroscopy of glycerol. Journal of Raman Spectroscopy, 2000. 31(12): p. 1121-1126.

171. De Gelder, J., et al., Reference database of Raman spectra of biological molecules. Journal of Raman Spectroscopy, 2007. 38(9): p. 1133-1147.

172. Han, J., et al., Co-electrospun blends of PLGA, gelatin, and elastin as potential nonthrombogenic scaffolds for vascular tissue engineering. Biomacromolecules, 2011. 12(2): p. 399-408.

173. Higdon, J. and V. Drake, An evidence-based approach to vitamins and minerals: health benefits and intake recommendations. 2011: Thieme Medical Pub.

174. Wollensak, G., et al., Collagen fiber diameter in the rabbit cornea after collagen crosslinking by riboflavin/UVA. Cornea, 2004. 23(5): p. 503.

175. Raiskup-Wolf, F., et al., Collagen crosslinking with riboflavin and ultraviolet-A light in keratoconus: long-term results. Journal of Cataract \& Refractive Surgery, 2008. 34(5): p. 796-801.

176. Yi, X., et al., Porous chitosan scaffold and ngf promote neuronal differentiation of neural stem cells in vitro. Neuro endocrinology letters, 2010. 32(5): p. 705-710.

177. Hersel, U., C. Dahmen, and H. Kessler, RGD modified polymers: biomaterials for stimulated cell adhesion and beyond. Biomaterials, 2003. 24(24): p. 4385-4415.

178. Linker, R.A., et al., Fumaric acid esters exert neuroprotective effects in neuroinflammation via activation of the Nrf2 antioxidant pathway. Brain, 2011. 134(3): p. 678-692. 
179. Koh, H., et al., Enhancement of neurite outgrowth using nano-structured scaffolds coupled with laminin. Biomaterials, 2008. 29(26): p. 3574-3582.

180. Kijeńska, E., et al., Interaction of Schwann cells with laminin encapsulated PLCL core-shell nanofibers for nerve tissue engineering. European Polymer Journal, 2014. 50: p. 30-38.

181. Binan, L., et al., Differentiation of neuronal stem cells into motor neurons using electrospun poly-L-lactic acid/gelatin scaffold. Biomaterials, 2014. 35(2): p. 664-674.

182. Han, J., et al., Co-electrospun blends of PLGA, gelatin, and elastin as potential nonthrombogenic scaffolds for vascular tissue engineering. Biomacromolecules, 2010. 12(2): p. 399-408.

183. Renoncourt, Y., et al., Neurons derived in vitro from ES cells express homeoproteins characteristic of motoneurons and interneurons. Mechanisms of development, 1998. 79(1): p. 185-197.

184. Wichterle, $\mathrm{H}$., et al., Directed differentiation of embryonic stem cells into motor neurons. Cell, 2002. 110(3): p. 385-397.

185. Deshpande, D.M., et al., Recovery from paralysis in adult rats using embryonic stem cells. Annals of neurology, 2006. 60(1): p. 32-44.

186. Hamada, M., et al., Introduction of the MASH1 gene into mouse embryonic stem cells leads to differentiation of motoneuron precursors lacking Nogo receptor expression that can be applicable for transplantation to spinal cord injury. Neurobiology of disease, 2006. 22(3): p. 509-522.

187. López-González, R., P. Kunckles, and I. Velasco, Transient recovery in a rat model of familial amyotrophic lateral sclerosis after transplantation of motor neurons derived from mouse embryonic stem cells. Cell transplantation, 2009. 18(10-11): p. 1171-1181.

188. Wu, C.-Y., et al., Proteomic assessment of a cell model of spinal muscular atrophy. BMC neuroscience, 2011. 12(1): p. 25. 


\section{VITA}

\section{XIZI DAI}

Born, Changsha, Hunan, China

2004-2008

B.S., Biomedical Engineering

Jiangsu University

Zhenjiang, China

2009-present

Ph.D. Candidate, Biomedical Engineering

Expected Graduation: May, 2015

Florida International University

Miami, Florida

\section{PUBLICATIONS AND PRESENTATIONS}

Dai, X., Kathiria, K., and Huang, Y.C. Biocompatibility study of Electrospun Micro-to-Nanometer fibers of Poly (glycerol dodecanoate). NanoFlorida, Miami, 2011 (Oral presentation)

Dai, X., Huang, Y.C. Preparation of Electrospun Fibers from Poly (glycerol dodecanoate co-fumarate) and their Cell Adhesion Studies. TERMIS, Houston, 2011 (Poster presentation)

Dai, X., Kathiria, K., and Huang, Y.C. A Novel Method for Electrospinning Long Fibers using Poly (glycerol dodecanoate). BMES, Hartford, 2011 (Poster presentation)

Dai, X., Huang, Y.C. Electrospun micro-to-nanometer fibers of poly (glycerol dodecanoate co-fumarate) and their potential for neural tissue engineering. NanoBio Collaborative International Conference ,Tampa, 2012 (Oral presentation)

Dai, X., Huang, Y.C. Electrospun Fibers of Poly (glycerol dodecanoate cofumarate) for Neural Tissue Engineering. BMES, Atlanta, 2012 (Poster presentation)

Dai, X., Huang, Y.C. Differentiation of Embryonic Stem Cells into Motor Neurons on Electrospun scaffolds. ISSCR, Boston, MA 2013 (Poster presentation) 
Dai, X., Huang, Y.C. Pluripotent Stem Cell Derived Neural Lineage Cells and Biomaterials for Neuroscience and Neuroengineering [J]. Journal of Neuroscience and Neuroengineering, 2013, 2(2): 119-140.

Dai, X., Kathiria, K., and Huang, Y.C. Electrospun fiber scaffolds of poly (glycerol-dodecanedioate) and its gelatin blended polymers for soft tissue engineering. Biofabrication, 2014, 6 (3): 035005.

Dai, X., Huang, Y.C. Electrospun Fibrous Scaffolds of Poly (glyceroldodecanedioate) for Engineering Neural Tissues from Mouse Embryonic Stem Cells. Journal of Visualized Experiments,doi:10.3791/51587 (2014)

Dai, X., Leichner, J., Huang, Y.C., and Lin, W.C. Efficient and Low-Cost Differentiation of Embryonic Stem Cells into Motor Neurons by Metabolites (2015). Submitted.

Dai, X., Leichner, J., Huang, Y.C., and Lin, W.C. Peptides modified polymer poly (glycerol- dodecanedioate co-fumarate) for efficient control of motor neurons differentiation. (2015). Submitted.

Leichner, J., Dai, X., Huang, Y.C., and Lin, W.C. The Metabolite Approach: Novel and Low-Cost Derivation of Cardiomyocytes from Mouse Embryonic Stem Cells (2015). Ready to submit. 